Pure and Applied Mathematics Quarterly

Volume 9, Number 2

(Special Issue: In honor of

Dennis Sullivan, Part 2 of 2)

$189-289,2013$

\title{
Lagrangian Floer Theory over Integers: Spherically Positive Symplectic Manifolds
}

\author{
Kenji Fukaya, Yong-Geun Oh, Hiroshi Ohta, Kaoru Ono \\ Dedicate to Professor Dennis Sullivan on his seventieth birthday
}

\begin{abstract}
In this paper we study the Lagrangian Floer theory over $\mathbb{Z}$ or $\mathbb{Z}_{2}$. Under an appropriate assumption on ambient symplectic manifold, we show that the whole story of Lagrangian Floer theory in [6], [7] can be developed over $\mathbb{Z}_{2}$ coefficients, and over $\mathbb{Z}$ coefficients when Lagrangian submanifolds are relatively spin. The main technical tools used for the construction are the notion of the sheaf of groups, and stratification and compatibility of the normal cones applied to the Kuranishi structure of the moduli space of pseudo-holomorphic discs.

Keywords: Floer cohomology, Lagrangian submanifolds, orbifold, stack, stratified space, pseudo-holomorphic curve, spherically positive symplectic manifold.
\end{abstract}

\section{CONTEnTs}

1. Introduction

2. Kuranishi structure: review

3. Statement of the main technical result

Received May 25, 2011.

KF is supported partially by JSPS Grant-in-Aid for Scientific Research No.18104001 and Global COE program G08, YO by US NSF grant \# 0904197, HO by JSPS Grant-in-Aid for Scientific Research No.23340015, and KO by JSPS Grant-in-Aid for Scientific Research, No.21244002. 
4. Sheaves of group-category

5. The stack structure of the singular locus

6. The normal bundle of the singular locus and $C^{\infty}$ local triviality 214

7. Single valued piecewise smooth section of orbi-bundle: statement

8. System of tubular neighborhoods

9. Single valued piecewise smooth section of orbi-bundle: proof

10. Single valued perturbation of a space with Kuranishi structure: proof of Theorem 3.1

11. Moduli space of pseudo-holomorphic discs: review 238

12. Estimate of the virtual dimension of the singular locus

13. Filtered $A_{\infty}$ algebra over $\mathbb{Z}_{2}$ 254

13.1. Construction of a filtered $A_{n, K}$ structure over $\mathbb{Z}_{2}$

13.2. Construction of a filtered $A_{n, K}$ homomorphism over $\mathbb{Z}_{2}$

13.3. From $A_{n, K}$ structure to $A_{\infty}$ structure 266

14. The case of $\mathbb{Z}$ coefficients

15. How the results of [6], [7] are generalized to $\mathbb{Z}$ or $\mathbb{Z}_{2}$ coefficients 273

15.1. Statements

15.2. Proof of Theorems 1.3 and 1.4

15.3. Maurer-Cartan moduli space over $\Lambda_{0 \text {,nov }}^{R}$

15.4. Hochschild homology, operator $\mathfrak{p}^{\prime}$ and degeneration of the spectral sequence 


\section{INTRODUCTION}

In this paper we establish Floer theory over $\mathbb{Z}_{2}$ (resp. $\mathbb{Z}$ ) for a Lagrangian submanifold (resp. relatively spin Lagrangian submanifold) under the assumption that the ambient symplectic manifold is spherically positive. Here we define:

Definition 1.1. Let $J$ be an almost complex structure on $M$. We call $J$ spherically positive if every $J$-holomorphic sphere $v: S^{2} \rightarrow M$ with $c_{1}(M)[v] \leq 0$ is constant.

For a symplectic manifold $(M, \omega)$, we denote by $\mathcal{J}_{(M, \omega)}^{c_{1}>0}$ the set of spherically positive almost complex structures which are compatible with $\omega$.

We call a symplectic manifold $(M, \omega)$ spherically positive if there exists a compatible spherically positive almost complex structure $J$. Sometimes we say $(M, \omega, J)$ is spherically positive.

Throughout this paper all symplectic manifolds are assumed to be compact or tame. We always assume that Lagrangian submanifolds are compact.

Example 1.2. (1) We recall that a symplectic manifold $(M, \omega)$ is (positively spherically) monotone if there exists $c>0$ such that $[\omega](\alpha)=c c_{1}(M)(\alpha)$ for any $\alpha \in \pi_{2}(M)$. It is easy to see that any monotone symplectic manifolds are spherically positive. For example, $\mathbb{C} P^{n}, \mathbb{C}^{n}, T^{2 n}$ are spherically positive with respect to the standard complex structure.

(2) If $M$ is a Fano manifold, $(M, \omega)$ is spherically positive for any Kähler form $\omega$.

(3) Any 4-dimensional symplectic manifold is spherically positive with respect to generic almost complex structures.

(4) Any product of spherically positive symplectic manifolds is spherically positive.

Remark 1.3. The set $\mathcal{J}_{(M, \omega)}^{c_{1}>0}$ may be neither path connected nor dense in the set of almost complex structures compatible with $\omega$, except when $\operatorname{dim} M=4$ or $M$ is monotone.

If $\psi:(M, \omega) \rightarrow\left(M^{\prime}, \omega^{\prime}\right)$ is a symplectic diffeomorphism, it induces a bijection $\psi_{*}: \mathcal{J}_{(M, \omega)}^{c_{1}>0} \rightarrow \mathcal{J}_{\left(M^{\prime}, \omega^{\prime}\right)}^{c_{1}>0}$ in an obvious way. 
Our main result in this paper is as follows. For a commutative ring $R$ with unit we define the universal Novikov ring $\Lambda_{0, \text { nov }}^{R}$ over $R$ by

$$
\begin{aligned}
\Lambda_{\text {nov }}^{R} & =\left\{\sum_{i=0}^{\infty} a_{i} T^{\lambda_{i}} e^{\mu_{i}} \mid a_{i} \in R, \mu_{i} \in \mathbb{Z}, \lambda_{i} \in \mathbb{R}, \lim _{i \rightarrow \infty} \lambda_{i}=+\infty\right\}, \\
\Lambda_{0, \text { nov }}^{R} & =\left\{\sum_{i=0}^{\infty} a_{i} T^{\lambda_{i}} e^{\mu_{i}} \in \Lambda_{\text {nov }} \mid \lambda_{i} \geq 0\right\} .
\end{aligned}
$$

Here $T, e$ are indeterminates. We define a filtration $F^{\lambda} \Lambda_{\text {nov }}^{R}=\left\{\sum_{i} a_{i} T^{\lambda_{i}} e^{\mu_{i}} \mid \lambda_{i} \geq\right.$ $\lambda$ \} and a degree $\operatorname{deg}\left(a T^{\lambda} e^{\mu}\right)=2 \mu$ for $a T^{\lambda} e^{\mu} \in \Lambda_{\text {nov }}^{R}$. Then $\Lambda_{0, \text { nov }}^{R}$ and $\Lambda_{\text {nov }}^{R}$ become filtered graded commutative rings. We denote by $\Lambda_{0, \text { nov }}^{+, R}$ the ideal of $\Lambda_{0, \text { nov }}^{R}$ that consists of elements such that $\lambda_{i}>0$.

Theorem 1.1. Let $(M, \omega, J)$ be a spherically positive symplectic manifold and $L$ its Lagrangian submanifold.

(1) We can associate a structure of filtered $A_{\infty}$ algebra $\left\{\mathfrak{m}_{k}^{J}\right\}_{k=0}^{\infty}$ on $H^{*}\left(L ; \Lambda_{0, \text { nov }}^{\mathbb{Z}_{2}}\right)$, which depends only on the connected component of $\mathcal{J}_{(M, \omega)}^{c_{1}>0}$ containing $J$ up to isomorphism.

(2) If $\psi:(M, L) \rightarrow\left(M^{\prime}, L^{\prime}\right)$ is a symplectic diffeomorphism, we can associate to it an isomorphism $\psi_{*}:\left(H^{*}\left(L ; \Lambda_{0, \text { nov }}^{\mathbb{Z}_{2}}\right),\left\{\mathfrak{m}_{k}^{J}\right\}\right) \rightarrow$ $\left(H^{*}\left(L^{\prime} ; \Lambda_{0, \text { nov }}^{\mathbb{Z}_{2}}\right),\left\{\mathfrak{m}_{k}^{\psi_{*} J}\right\}\right)$ of filtered $A_{\infty}$ algebras whose homotopy class depends only on the isotopy class of symplectic diffeomorphism $\psi:(M, L) \rightarrow$ $\left(M^{\prime}, L^{\prime}\right)$. Moreover $\left(\psi \circ \psi^{\prime}\right)_{*}=\psi_{*} \circ \psi_{*}^{\prime}$.

(3) The Poincaré dual $P D([L]) \in H^{0}\left(L ; \Lambda_{0, \text { nov }}^{\mathbb{Z}_{2}}\right)$ of the fundamental class $[L]$ is the unit of our filtered $A_{\infty}$ algebra. The homomorphism $\psi_{*}$ is unital.

(4) If $L$ is relatively spin and the homology group $H(L ; \mathbb{Z})$ is a torsion free $\mathbb{Z}$ module, the above holds for the coefficient ring $\Lambda_{0, \text { nov }}^{\mathbb{Z}}$ in place of $\Lambda_{0, \text { nov }}^{\mathbb{Z}_{2}}$. The tensor product of this filtered $A_{\infty}$ algebra with $\mathbb{Q}$ becomes unitally homotopy equivalent to the filtered $A_{\infty}$ algebra of Theorem $A$ [6].

Remark 1.4. We note that in Theorem 1.1 we put some assumption on the symplectic manifold $(M, \omega)$ but not on its Lagrangian submanifold $L$. For example, Theorem 1.1 applies to any compact Lagrangian submanifold in $\mathbb{C}^{n}$.

Remark 1.5. In case $L$ is relatively spin but the homology group $H(L ; \mathbb{Z})$ is not free, we can prove a slightly different but a similar result. See Section 14. The same remark applies to Theorem 1.2. 
Let $L_{1}, L_{0}$ be Lagrangian submanifolds of $(M, \omega)$. We assume that they have clean intersection in the following sense.

Definition 1.6. A pair of submanifolds $L_{1}, L_{0}$ of a manifold $M$ is said to have clean intersection if $L_{1} \cap L_{0}$ is a smooth submanifold and

$$
T_{x} L_{1} \cap T_{x} L_{0}=T_{x}\left(L_{1} \cap L_{0}\right)
$$

for each $x \in L_{1} \cap L_{0}$.

We decompose

$$
L_{1} \cap L_{0}=\bigcup_{h} R_{h}
$$

to the connected components. Let $\mu_{L}\left(R_{h}\right)$ be the Bott-Morse version of Maslov index which is defined in Proposition 3.7 .59 (3.7.60.1), also p.146, [6]. Actually we need to fix an extra data, (denoted by $w$ there) to define the Bott-Morse version of Maslov index. [·] denotes the degree shift. We put:

$$
C\left(L_{1}, L_{0} ; \Lambda_{0, \text { nov }}^{\mathbb{Z}_{2}}\right)=\bigoplus_{h} H\left(R_{h} ; \mathbb{Z}_{2}\right)\left[\mu_{L}\left(R_{h}\right)\right] \otimes \Lambda_{0, \text { nov }}^{\mathbb{Z}_{2}}
$$

In case $L_{1}$ and $L_{0}$ are oriented, we defined in [7] Subsection 8.8 a local system $\Theta_{R_{h}}^{-}$on each of the connected component $R_{h}$ of $L_{1} \cap L_{0}$ by a homomorphism $: \pi_{1}\left(R_{h}\right) \rightarrow\{ \pm 1\}=\operatorname{Aut}(\mathbb{Z})$. We then put

$$
C\left(L_{1}, L_{0} ; \Lambda_{0, \text { nov }}^{\mathbb{Z}}\right)=\bigoplus_{h} H\left(R_{h} ; \Theta_{R_{h}}^{-}\right)\left[\mu_{L}\left(R_{h}\right)\right] \otimes \Lambda_{0, \text { nov }}^{\mathbb{Z}} .
$$

Theorem 1.2. Let $L_{1}, L_{0}$ be a pair of Lagrangian submanifolds of $M$.

(1) $C\left(L_{1}, L_{0} ; \Lambda_{0, \text { nov }}^{\mathbb{Z}_{2}}\right)$ has a structure of unital filtered $A_{\infty}$ bimodule over the pair

$$
\left(\left(H\left(L_{1} ; \Lambda_{0, \text { nov }}^{\mathbb{Z}_{2}}\right),\left\{\mathfrak{m}_{k}^{J}\right\}_{k=0}^{\infty}\right),\left(H\left(L_{0} ; \Lambda_{0, \text { nov }}^{\mathbb{Z}_{2}}\right),\left\{\mathfrak{m}_{k}^{J}\right\}_{k=0}^{\infty}\right)\right) .
$$

(2) This depends only on the connected component of $J \in \mathcal{J}_{(M, \omega)}^{c_{1}>0}$ up to an isomorphism of unital filtered $A_{\infty}$ bimodule.

(3) A similar functoriality as Theorem 1.1 (2) holds.

If $\left(L_{0}, L_{1}\right)$ is a relatively spin pair and $H\left(L_{1} \cap L_{0} ; \Theta_{R_{h}}^{-}\right)$is a free $\mathbb{Z}$ module, we can take $\Lambda_{0, \text { nov }}^{\mathbb{Z}}$ as a coefficient ring in place of $\Lambda_{0, \text { nov }}^{\mathbb{Z}_{2}}$. This bimodule is homotopy equivalent to the ones in [6] Theorem $F$ after taking $\otimes \mathbb{Q}$. 
We can generalize various other results of [6], [7] to ones over $\Lambda_{0 \text {,nov }}^{\mathbb{Z}_{2}}$ or $\Lambda_{0, \text { nov }}^{\mathbb{Z}}$ in a similar way. See Section 15 for the precise statement.

In [6], [7] we worked over $\Lambda_{0, \text { nov }}^{\mathbb{Q}}$ because we used multivalued perturbations (multisections) to define (virtual) fundamental chains of various moduli spaces involved in the construction. We need a multisection to perturb the moduli space to achieve transversality since in general there is a nontrivial automorphism of elements of the moduli spaces involved. More precisely, the moduli space we use is the stable map compactification $\mathcal{M}_{k+1}^{\text {main }}(\beta)$ of the space of pseudo-holomorphic discs with $k+1$ boundary marked points.

In order to prove Theorems 1.1 and 1.2 we need to use single valued sections in place of multisections that satisfy some transversality properties. The space $\mathcal{M}_{k+1}^{\text {main }}(\beta)$ is regarded locally as the zero set of a section of an orbi-bundle over an orbifold. Transversality of the zero set in general fails even for a generic section of an orbi-bundle over an orbifold. Our main technical result to prove Theorems 1.1 and 1.2 is stated in Section 3. (Theorem 3.1.)

We next mention some of applications of Theorems 1.1 and 1.2. Since many of them are straightforward generalization of the results proved over $\mathbb{Q}$ coefficients in $[6],[7]$ we mention only a few of them below.

Theorem 1.3. Let $L$ be a compact Lagrangian submanifold of $\mathbb{C}^{n}$ that satisfies $H^{2}\left(L ; \mathbb{Z}_{2}\right)=0$. Then its Maslov class $\mu_{L} \in H^{1}(L ; \mathbb{Z})$ is nonzero.

Theorem 1.3 is proved in [6] under a similar but different assumption that $H^{2}(L ; \mathbb{Q})=0$ and $L$ is relatively spin (Theorem $\left.\mathrm{K}\right)$.

Theorem 1.4. Let $L \subset(M, \omega)$ be a Lagrangian submanifold such that the Maslov index homomorphism $\mu_{L}: \pi_{2}(M, L) \rightarrow \mathbb{Z}$ is trivial. Assume $H^{2}\left(L ; \mathbb{Z}_{2}\right)=0$ and $M$ is spherically positive. Then for any Hamiltonian diffeomorphism $\phi: M \rightarrow M$, we have

$$
L \cap \phi(L) \neq \emptyset .
$$

Moreover if $L$ is transversal to $\phi(L)$, there exists $p \in L \cap \phi(L)$ whose Maslov index is 0 .

Theorem 1.4 is a $\mathbb{Z}_{2}$ coefficients version of Theorem L [6].

The main new part of this paper which consists of Sections 3-10 is written in a way independent of [6],[7]. Actually the main result, Theorem 3.1, holds for an 
arbitrary space with Kuranishi structure with tangent bundle, which may or may not be related to the moduli space of pseudo-holomorphic curve. (We would like to recall the readers that the theory of Kuranishi structures itself that appeared in 1996 is not restricted to but independent of the study of pseudo-holomorphic curves, although its main application so far is aimed to the study of moduli spaces of pseudo-holomorphic curves. )

Sections 11 and after are devoted to the generalizations of the results of [6], $[7]$ to the $\mathbb{Z}$ or $\mathbb{Z}_{2}$ coefficients. So they necessarily use various results from [6], [7]. We, however, try to make them separately readable without reading [6], [7] modulo the details of the proofs, as much as possible. Especially Section 13, which constructs a filtered $A_{\infty}$ algebra over $\mathbb{Z}_{2}$, we repeat the construction of compatible sections carried out in the transversality part (Section 7.2) of [7]. There we use the results on analysis and homological algebra (construction of Kuranishi structure on moduli space of holomorphic discs), which we refer readers to [7].

This paper is one third of [8], that is 'Chapter 8' of the 2006 preprint version of $[6],[7]$. ('Chapter 8 ' is one of the two chapters which were removed from [6], [7] when it was finally published. We removed them to keep the size of [6], [7] within the requirement of the publisher.) During 5 years there are some progress. For example, the readers can see such progress in Subsections 15.3, 15.4.

Another one third of [8] becomes [12]. The paper containing the rest of [8], which proves Arnold-Givental conjecture in spherically positive case, is in preparation.

\section{Kuranishi structure: RevieW}

In this section, we review the definition of Kuranishi structure. There is nothing new in this section except Definitions 2.7 and 2.8, which we use in later sections. The main purpose of this section is a review and fixing notations. We refer [14] and Section A1 of [7]. Let $X$ be a compact metrizable space and $p \in X$.

Definition 2.1. A Kuranishi neighborhood of $p$ in $X$ is a quintet $\left(V_{p}, E_{p}, \Gamma_{p}, \psi_{p}, s_{p}\right)$ such that:

(i) $V_{p}$ is a smooth manifold of finite dimension, which may or may not have boundary or corner.

(ii) $E_{p}$ is a real vector space of finite dimension. 
(iii) $\Gamma_{p}$ is a finite group acting smoothly and effectively on $V_{p}$ and has a linear representation on $E_{p}$.

(iv) $s_{p}$ is a $\Gamma_{p}$ equivariant smooth map $V_{p} \rightarrow E_{p}$.

(v) $\psi_{p}$ is a homeomorphism from $s_{p}^{-1}(0) / \Gamma_{p}$ to a neighborhood of $p$ in $X$.

We put $U_{p}=V_{p} / \Gamma_{p}$ and say that $U_{p}$ is a Kuranishi neighborhood. We sometimes say that $V_{p}$ is a Kuranishi neighborhood by an abuse of notation.

We call $E_{p} \times V_{p} \rightarrow V_{p}$ the obstruction bundle and $s_{p}$ the Kuranishi map. For $x \in V_{p}$, denote by $I_{x}$ the isotropy subgroup at $x$, i.e.,

$$
I_{x}=\left\{\gamma \in \Gamma_{p} \mid \gamma x=x\right\} .
$$

Let us take a point $o_{p} \in V_{p}$ with $s_{p}\left(o_{p}\right)=0$ and $\psi\left(\left[o_{p}\right]\right)=p$. We may and will assume that $o_{p}$ is fixed by all elements of $\Gamma_{p}$.

Definition 2.2. Let $\left(V_{p}, E_{p}, \Gamma_{p}, \psi_{p}, s_{p}\right),\left(V_{q}, E_{q}, \Gamma_{q}, \psi_{q}, s_{q}\right)$ be Kuranishi neighborhoods of $p \in X$ and $q \in \psi_{p}\left(s_{p}^{-1}(0) / \Gamma_{p}\right)$, respectively. We say a triple $\left(\hat{\phi}_{p q}, \phi_{p q}, h_{p q}\right)$ a coordinate change if

(i) $h_{p q}$ is an injective homomorphism $\Gamma_{q} \rightarrow \Gamma_{p}$.

(ii) $\phi_{p q}: V_{p q} \rightarrow V_{p}$ is an $h_{p q}$ equivariant smooth embedding from a $\Gamma_{q}$ invariant open neighborhood $V_{p q}$ of $o_{q}$ to $V_{p}$, such that the induced map $\underline{\phi}_{p q}: V_{p q} / \Gamma_{q} \rightarrow V_{p} / \Gamma_{p}$ is injective.

(iii) $\left(\hat{\phi}_{p q}, \phi_{p q}\right)$ is an $h_{p q}$ equivariant embedding of vector bundles $E_{q} \times V_{p q} \rightarrow$ $E_{p} \times V_{p}$

(iv) $\hat{\phi}_{p q} \circ s_{q}=s_{p} \circ \phi_{p q}$. Here and hereafter we sometimes regard $s_{p}$ as a section $s_{p}: V_{p} \rightarrow E_{p} \times V_{p}$ of trivial bundle $E_{p} \times V_{p} \rightarrow V_{p}$.

(v) $\psi_{q}=\psi_{p} \circ \underline{\phi}_{p q}$ on $\left(s_{q}^{-1}(0) \cap V_{p q}\right) / \Gamma_{q}$. Here $\underline{\phi}_{p q}$ is as in (ii).

(vi) $h_{p q}$ restricts to an isomorphism $\left(\Gamma_{q}\right)_{x} \rightarrow\left(\Gamma_{p}\right)_{\phi_{p q}(x)}$ for any $x \in V_{p q}$.

Definition 2.3. A Kuranishi structure on $X$ assigns a Kuranishi neighborhood $\left(V_{p}, E_{p}, \Gamma_{p}, \psi_{p}, s_{p}\right)$ for each $p \in X$ and a coordinate change $\left(\hat{\phi}_{p q}, \phi_{p q}, h_{p q}\right)$ for each $q \in \psi_{p}\left(s_{p}^{-1}(0) / \Gamma_{p}\right)$ such that the following holds.

(i) $\operatorname{dim} V_{p}-\operatorname{rank} E_{p}$ is independent of $p$.

(ii) If $r \in \psi_{q}\left(\left(s_{q}^{-1}(0) \cap V_{p q}\right) / \Gamma_{q}\right), q \in \psi_{p}\left(s_{p}^{-1}(0) / \Gamma_{p}\right)$, then there exists $\gamma_{p q r}^{\alpha} \in \Gamma_{p}$ for each connected component $\alpha$ of $\phi_{q r}^{-1}\left(V_{p q}\right) \cap V_{q r} \cap V_{p r}$ such that

$h_{p q} \circ h_{q r}=\gamma_{p q r}^{\alpha} \cdot h_{p r} \cdot\left(\gamma_{p q r}^{\alpha}\right)^{-1}, \quad \phi_{p q} \circ \phi_{q r}=\gamma_{p q r}^{\alpha} \cdot \phi_{p r}, \quad \hat{\phi}_{p q} \circ \hat{\phi}_{q r}=\gamma_{p q r}^{\alpha} \cdot \hat{\phi}_{p r}$. 
Here the second equality holds on the connected component $\alpha$ of $\phi_{q r}^{-1}\left(V_{p q}\right) \cap$ $V_{q r} \cap V_{p r}$ and the third equality holds on the restriction of $E_{r} \times\left(\phi_{q r}^{-1}\left(V_{p q}\right) \cap\right.$ $\left.V_{q r} \cap V_{p r}\right)$ to $\alpha$. In case $V_{p}$ has boundary or corners, we say that $\left(V_{p}, E_{p}, \Gamma_{p}, \psi_{p}, s_{p}\right)$ is a Kuranishi structure with boundary or corner.

We remark that (ii) is equivalent to the condition that

$$
\underline{\phi}_{p q} \circ \underline{\phi}_{q r}=\underline{\phi}_{p r} .
$$

(We can prove this equivalence by using the effectivity of the $\Gamma_{p}$ action.)

We call $\operatorname{dim} V_{p}-\operatorname{rank} E_{p}$ the virtual dimension (or dimension) of the Kuranishi structure.

In case $K \subset X$ we say $U$ is a Kuranishi neighborhood of $K$ if $U=\left\{V_{p_{i}} / \Gamma_{p_{i}}\right\}$, $K \subset \bigcup_{i} \psi_{p_{i}}\left(s_{p_{i}}^{-1}(0) / \Gamma_{p_{i}}\right)$.

An orbifold structure on $X$ is, by definition, a Kuranishi structure on $X$ such that $E_{p}=0$ for all $p$.

We recall:

Lemma 2.1. ([[14], Lemma 6.3]) Let $X$ be a space with Kuranishi structure. Then there exists a finite set $P \subset X$, a partial order $<$ on $P$, and a Kuranishi neighborhood $\left(V_{p}, E_{p}, \Gamma_{p}, \psi_{p}, s_{p}\right)$ of $p$ for each $p \in P$, with the following properties.

(i) If $q<p, \psi_{p}\left(s_{p}^{-1}(0) / \Gamma_{p}\right) \cap \psi_{q}\left(s_{q}^{-1}(0) / \Gamma_{q}\right) \neq \emptyset$, then there exists $\left(V_{p q}, \hat{\phi}_{p q}, \phi_{p q}, h_{p q}\right)$ where:

(a) $V_{p q}$ is a $\Gamma_{q}$ invariant open subset of $V_{q}$ such that $V_{p q} / \Gamma_{q}$ contains

$$
\psi_{q}^{-1}\left(\psi_{p}\left(s_{p}^{-1}(0) / \Gamma_{p}\right) \cap \psi_{q}\left(s_{q}^{-1}(0) / \Gamma_{q}\right)\right),
$$

(b) $h_{p q}$ is an injective homomorphism $\Gamma_{q} \rightarrow \Gamma_{p}$ with its image $\left(\Gamma_{p}\right)_{\phi_{p q}(q)}$,

(c) $\phi_{p q}: V_{p q} \rightarrow V_{p}$ is an $h_{p q}$ equivariant smooth embedding such that the induced map $V_{p q} / \Gamma_{q} \rightarrow V_{p} / \Gamma_{p}$ is injective,

(d) $\left(\hat{\phi}_{p q}, \phi_{p q}\right)$ is an $h_{p q}$ equivariant embedding of vector bundles $E_{q} \times V_{p q} \rightarrow$ $E_{p} \times V_{p}$

(e) $\hat{\phi}_{p q} \circ s_{q}=s_{p} \circ \phi_{p q}, \quad \psi_{q}=\psi_{p} \circ \underline{\phi}_{p q}$.

(ii) If $r<q<p, \psi_{p}\left(s_{p}^{-1}(0) / \Gamma_{p}\right) \cap \psi_{q}\left(s_{q}^{-1}(0) / \Gamma_{q}\right) \cap \psi_{r}\left(s_{r}^{-1}(0) / \Gamma_{r}\right) \neq \emptyset$, then there exists $\gamma_{p q r}^{\alpha} \in \Gamma_{p}$ for each connected component $\alpha$ of $\phi_{q r}^{-1}\left(V_{p q}\right) \cap V_{q r} \cap V_{p r}$ such 
that

$h_{p q} \circ h_{q r}=\gamma_{p q r}^{\alpha} \cdot h_{p r} \cdot\left(\gamma_{p q r}^{\alpha}\right)^{-1}, \quad \phi_{p q} \circ \phi_{q r}=\gamma_{p q r}^{\alpha} \cdot \phi_{p r}, \quad \hat{\phi}_{p q} \circ \hat{\phi}_{q r}=\gamma_{p q r}^{\alpha} \cdot \hat{\phi}_{p r}$.

Here the second equality holds on the connected component $\alpha$ of $\phi_{q r}^{-1}\left(V_{p q}\right) \cap$ $V_{q r} \cap V_{p r}$, and the third equality holds on the restriction to $\alpha$ of $E_{r} \times$ $\left(\phi_{q r}^{-1}\left(V_{p q}\right) \cap V_{q r} \cap V_{p r}\right)$.

(iii) We have $\bigcup_{p \in P} \psi_{p}\left(s_{p}^{-1}(0) / \Gamma_{p}\right)=X$.

(iv) If $\psi_{p}\left(s_{p}^{-1}(0) / \Gamma_{p}\right) \cap \psi_{q}\left(s_{q}^{-1}(0) / \Gamma_{q}\right) \neq \emptyset$, then either $p<q$ or $q<p$.

We call the system of Kuranishi neighborhood and coordinate change

$$
\left(\left\{\left(V_{p}, E_{p}, \Gamma_{p}, \psi_{p}, s_{p}\right) \mid p \in P\right\},\left\{\left(V_{p q}, \hat{\phi}_{p q}, \phi_{p q}, h_{p q}\right) \mid p, q \in P, p<q\right\}\right)
$$

in Lemma 2.1 the good coordinate system. (Note we require existence of $\gamma_{p q r}^{\alpha}$ but do not include it as a part of the structure. Compare Remark 4.5 (iv).)

Definition 2.4. Consider the situation of Lemma 2.1. Let $Y$ be a topological space. A family $\left\{f_{p}\right\}$ of $\Gamma_{p}$-equivariant continuous maps $f_{p}: V_{p} \rightarrow Y$ is said to be a strongly continuous map if

$$
f_{p} \circ \phi_{p q}=f_{q}
$$

on $V_{p q}$. A strongly continuous map induces a continuous map $f: X \rightarrow Y$. We will ambiguously denote $f=\left\{f_{p}\right\}$ when the meaning is clear.

When $Y$ is a smooth manifold, a strongly continuous map $f: X \rightarrow Y$ is defined to be smooth if all $f_{p}: V_{p} \rightarrow Y$ are smooth. We say that it is weakly submersive if each of $f_{p}$ is a submersion.

When $Y$ is a simplicial complex, we say $f=\left\{f_{p}\right\}$ is strongly piecewise smooth if each of $f_{p}$ is piecewise smooth.

Consider the situation of Lemma 2.1. We identify a neighborhood of $\phi_{p q}\left(V_{p q}\right)$ in $V_{p}$ with a neighborhood of the zero section of the normal bundle $N_{V_{p q}} V_{p} \rightarrow V_{p q}$, using an exponential map of an appropriate Riemannian metric. We take the fiber derivative of the Kuranishi map $s_{p}$ along the fiber direction and obtain a homomorphism

$$
d_{\text {fiber }} s_{p}: N_{V_{p q}} V_{p} \rightarrow E_{p} \times V_{p q}
$$


which is an $h_{p q}$-equivariant bundle homomorphism. Note we need to take and fix a connection on the bundle $E_{p}$ to define (3). However (3) is independent of the choice of the connection on the zero set of $s_{p}$.

Definition 2.5. We say that the space with Kuranishi structure $X$ has a tangent bundle if $d_{\mathrm{fiber}} s_{p}$ induces a bundle isomorphism

$$
N_{V_{p q}} V_{p} \cong \frac{E_{p} \times V_{p q}}{\hat{\phi}_{p q}\left(E_{q} \times V_{p q}\right)}
$$

as $\Gamma_{q}$-equivariant bundles on $V_{p q} \cap s_{p}^{-1}(0)$.

By definition, the following diagram commutes for each $x \in \phi_{q r}^{-1}\left(V_{p q}\right) \cap V_{q r} \cap V_{p r}$.

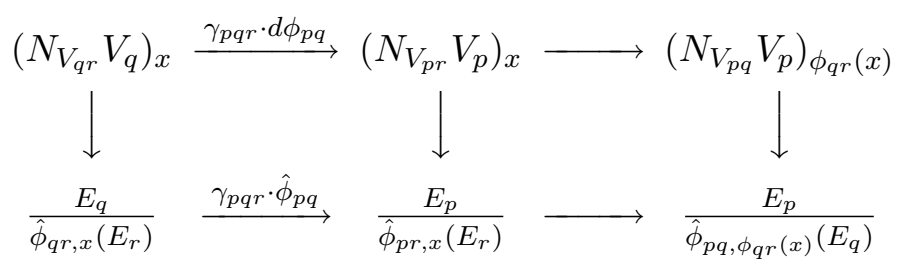

Here and hereafter $\hat{\phi}_{q p, x}: E_{q} \rightarrow E_{p}$ is the restriction of the bundle map $\hat{\phi}_{p q}$ to the fiber of $x$. And we take and fix a connection on the bundle $E_{p}$ so that the image of $\hat{\phi}_{p q}$ is invariant under the parallel transport. Then (3) is well defined and (5) commutes.

We may shrink $V_{p q}$ to a smaller subset if necessary so that (4) is an isomorphism not only on $V_{p q} \cap s_{p}^{-1}(0)$ but also everywhere on $V_{p q}$. So hereafter we always assume so.

Definition 2.6. Let $X$ be a space with Kuranishi structure which has a tangent bundle. We say that the Kuranishi structure on $X$ is oriented if we have a trivialization of

$$
\Lambda^{\mathrm{top}} E_{p}^{*} \otimes \Lambda^{\mathrm{top}} T V_{p}
$$

which is compatible with isomorphism (4).

We next define the compatibility of sections of the obstruction bundles over various Kuranishi charts as follows.

Definition 2.7. Let us consider the situation of Lemma 2.1. We assume that our Kuranishi structure has a tangent bundle. Suppose $p, q \in P, q<p$ and suppose we have sections $s_{p}^{\prime}, s_{q}^{\prime}$ of $E_{p} \times V_{p}, E_{q} \times V_{q}$ respectively. We consider 
the embedding $\phi_{p q}: V_{p q} \rightarrow V_{p}$ in Lemma 2.1 (i) (c). We identify its normal bundle $N_{V_{p q}} V_{p}$ with a tubular neighborhood of $\phi_{p q}\left(V_{p q}\right)$. For each $x \in V_{p q}$ we fix a splitting

$$
E_{p} \cong \hat{\phi}_{p q, x}\left(E_{q}\right) \oplus \frac{E_{p}}{\hat{\phi}_{p q, x}\left(E_{q}\right)} .
$$

For each $y \in N_{V_{p q}} V_{p}$, we obtain an element $1(y)$ of $\frac{E_{p}}{\hat{\phi}_{p q, \pi(y)}\left(E_{q}\right)}$ by using the isomorphism (4). Then, on $N_{V_{p q}} V_{p}$, we define a section $s_{q}^{\prime} \oplus 1$ by

$$
\left(s_{q}^{\prime} \oplus 1\right)(y)=s_{q}^{\prime}(\pi(y)) \oplus 1(y) \in \hat{\phi}_{p q, x}\left(E_{q}\right) \oplus \frac{E_{p}}{\hat{\phi}_{p q, \pi(y)}\left(E_{q}\right)} \cong E_{p}^{n} .
$$

Here we use the splitting (6). Now we say that $s_{p}^{\prime}$ is compatible with $s_{q}^{\prime}$ if the restriction of $s_{p}^{\prime}$ to $N_{V_{p q}} V_{p}$ coincides with $s_{q}^{\prime} \oplus 1$.

We note that the section $s_{p}$ (that is a Kuranishi map) is compatible with $s_{q}$ in the sense defined above. This follows from the fact that (4) is induced by the fiber derivative of the Kuranishi map.

A global section $\mathfrak{s}^{\prime}=\left\{s_{p}^{\prime}\right\}_{p \in P}$ of the obstruction bundle of a Kuranishi structure is a compatible system of sections $\left(s_{p}^{\prime}: V_{p} \rightarrow E_{p}\right)$. A section $\mathfrak{s}^{\prime}=\left\{s_{p}^{\prime}\right\}_{p \in P}$ of the obstruction bundle of a Kuranishi structure is also called a global section if it is a compatible system of sections $\left(s_{p}^{\prime}: V_{p}^{\prime} \rightarrow E_{p}\right)$ after shrinking Kuranishi neighborhood $V_{p}$ to a relatively compact subspace $V_{p}^{\prime} \subset V_{p}$ appropriately. Actually in the following definition we shrink Kuranishi neighborhoods and consider the zero set of $s_{p}^{\prime}$ in the closure $\bar{V}_{p}^{\prime}$ to construct a topological space $X^{\prime}$.

Definition 2.8. In case $\mathfrak{s}^{\prime}=\left\{s_{p}^{\prime}\right\}_{p \in P}$ is a global section we define a topological space

$$
X^{\prime}=\left(\mathfrak{s}^{\prime}\right)^{-1}(0)
$$

as follows. We consider the disjoint union

$$
\bigcup_{p \in P} \frac{\left(s_{p}^{\prime}\right)^{-1}(0)}{\Gamma_{p}} \times\{p\}
$$

Let $\tilde{x} \in\left(s_{p}^{\prime}\right)^{-1}(0) \subset \bar{V}_{p}^{\prime}$ and $\tilde{y} \in\left(s_{q}^{\prime}\right)^{-1}(0) \subset \bar{V}_{q}^{\prime}$. We define $(x, p) \sim^{\prime}(y, q)$ if $q<p, x=[\tilde{x}], y=[\tilde{y}], \tilde{y} \in V_{p q}$ and

$$
\phi_{p q}(\tilde{y})=\tilde{x}
$$

Let $\sim$ be the equivalence relation on the set $(7)$ which is generated by $\sim^{\prime}$. We define $X^{\prime}$ as the set of equivalence classes of this equivalence relation. We put 
quotient topology on it. We have a Kuranishi structure on $X^{\prime}$ whose Kuranishi map is $s_{p}^{\prime}$ in an obvious way.

If $f$ is a strongly continuous map from $X$ to $Y$, it induces a strongly continuous map from $X^{\prime}$ to $Y$. The case of strongly smooth map or strongly piecewise smooth map is similar.

\section{Statement of the main technical Result}

Let $X$ be a space with Kuranishi structure. We fix its good coordinate system $\left(\left\{\left(V_{p}, E_{p}, \Gamma_{p}, \psi_{p}, s_{p}\right) \mid p \in P\right\},\left\{\left(V_{p q}, \hat{\phi}_{p q}, \phi_{p q}, h_{p q}\right) \mid p, q \in P, p<q\right\}\right)$.

Definition 3.1. Let $x \in X$. We take $p \in P$ and $\tilde{x} \in V_{p}$ such that $s_{p}(\tilde{x})=0$ and $\psi_{p}(\tilde{x})=x$. We put

$$
I_{x}=\left\{\gamma \in \Gamma_{p} \mid \gamma \tilde{x}=\tilde{x}\right\}
$$

and call it the isotropy group. It is easy to see that $I_{x}$ is independent of the choice of $p$ and $\tilde{x}$ and depends only on $x$.

For a finite group $\Gamma$, we define

$$
X^{\cong}(\Gamma)=\left\{x \in X \mid I_{x} \cong \Gamma\right\} .
$$

We decompse $X^{\cong}(\Gamma)$ into connected components

$$
X^{\cong}(\Gamma)=\bigcup_{i} X^{\cong}(\Gamma ; i) .
$$

Definition 3.2. We define the integers $d(X ; \Gamma ; i)$ as follows. Let $x \in X^{\cong}(\Gamma ; i)$. We take $p, \tilde{x}$ as in Definition 3.1. The group $I_{x}$ acts on $\left(E_{p}\right)_{\tilde{x}}$, the fiber of the obstruction bundle at $\tilde{x}$. We then put

$$
E_{\tilde{x}}^{\Gamma}=\left\{v \in E_{\tilde{x}} \mid \forall \gamma \in \Gamma \gamma v=v\right\} .
$$

Its dimension depends only on $\Gamma, i$ but independent of $p, \tilde{x}, x$. The group $\Gamma$ acts also on the tangent bundle $T_{\tilde{x}} V_{p}$. We put

$$
T_{\tilde{x}} V_{p}^{\Gamma}=\left\{T_{\tilde{x}} V_{p} \mid \forall \gamma \in \Gamma \gamma v=v\right\} .
$$

Its dimension depends only on $\Gamma, i$ but independent of $p, \tilde{x}, x$. We now define

$$
d(X ; \Gamma ; i)=\operatorname{dim} T_{\tilde{x}} V_{p}^{\Gamma}-\operatorname{dim} E_{\tilde{x}}^{\Gamma} .
$$


Now the main technical result used in the proof of Theorems 1.1 and 1.2 is the following:

Theorem 3.1. Let $X$ be a space with Kuranishi structure that has a tangent bundle. We take a good coordinate system. Then there exists a strongly piecewise smooth global section $\mathfrak{s}^{\prime}$, which is arbitrarily close to the original Kuranishi map in $C^{0}$ sense, such that the following holds for $X^{\prime}=\left(\mathfrak{s}^{\prime}\right)^{-1}(0)$ defined in Definition 2.8 and its Kuranishi structure:

(i) $X^{\prime}$ has a triangulation.

(ii) Each of $X^{\prime \cong}(\Gamma)$ is a simplicial subcomplex of $X^{\prime}$.

(iii) We have

$$
\operatorname{dim} X^{\prime \cong}(\Gamma) \leq \max _{i} d(X ; \Gamma ; i) .
$$

Moreover if $f: X \rightarrow Y$ is a strongly smooth map from $X$ to a manifold $Y$, we may choose the triangulation of $X^{\prime}$ so that $f$ induces a piecewise smooth map $X^{\prime} \rightarrow Y$.

We note that if $s_{p}^{\prime}$ is a (single valued) section on $V_{p} / \Gamma_{p}$, then at $\tilde{x} \in V_{p}$ with $I_{\tilde{x}}=\Gamma$, the value $s_{p}^{\prime}(\tilde{x})$ is necessarily in the $\Gamma=\Gamma_{\tilde{x}}$ fixed point set of $\left(E_{p}\right) \tilde{x}$. This implies that the dimension appearing in the right hand side of (iii) above is optimal.

In the situation where the space $X$ with Kuranishi structure is a moduli space of geometric origin, the number $d(X ; \Gamma ; i)$ can be identified with an appropriate equivariant index. Thus we can estimate this number under certain geometric assumption. In case $d(X ; \Gamma ; i)$ is strictly smaller than the virtual dimension of $X$ minus 1, Theorem 3.1 implies that $X$ has a virtual fundamental chain over $\mathbb{Z}$ or over $\mathbb{Z}_{2}$. (To define virtual fundamental chain over $\mathbb{Z}$ we need to include orientation.) This is the way, we prove Theorems 1.1, 1.2. See Section 13 for more detail.

The proof of Theorem 3.1 occupies Sections 4-10. A brief outline of the proof is in order.

We use the theory of stratified sets for our proof. The collection of $X(\Gamma ; i)$ 's for various $\Gamma, i$ defines a natural stratification of $X$ and the stratification extends to the Kuranishi neighborhood. We construct our global section by an induction on the Kuranishi neighborhood. We apply induction twice. For the first step we 
work on one Kuranishi neighborhood and then we use the partial order $<$ of $P$ to inductively construct $s_{p}^{\prime}$.

When we restrict ourselves to a single Kuranishi neighborhood, we deal with an orbifold and an orbi-bundle. We use the stratification of an orbifold by its isotropy group to construct $s_{p}^{\prime}$ on each of the Kuranishi chart. We use the normal bundle of the stratum and a conical extension of the section on one stratum to its neighborhood. In Section 6, we study normal bundle of a stratum. As we will observe in Example 4.1 Section 4, the stratum (the set of $x$ with given $I_{x}$ ) does not have the normal bundle in a usual sense. Actually it has some twisted stack structure and the normal bundle is well-defined as a bundle over this stack. We will explain this point in Sections 4-6.

To construct the section $s_{p}^{\prime}$ inductively we need to use compatibility between the normal bundles of various components. The story of compatible systems of normal bundles is classical and was used in the study of triangulation of algebraic sets. Especially J. Mather [20] gave a nice definition of compatibility which we use with minor modification. (See Section 8.) We use this in our construction of $s_{p}^{\prime}$ and triangulation of its zero set applied to each Kuranishi neighborhood in Section 9 .

The inductive construction over various Kuranishi charts is actually standard using a good coordinate system. We perform this construction in Section 10, where we complete the proof of Theorem 3.1.

\section{Sheaves of Group-CATEGory}

Let $X$ be an orbifold and $\Gamma$ a finite group. We define

$$
X^{\cong}(\Gamma)=\left\{x \in X \mid I_{x} \cong \Gamma\right\} .
$$

In this paper, we need to consider a normal bundle $N_{X \cong(\Gamma)} X$ of $X^{\cong}(\Gamma)$ in $X$. At first sight, one might expect that there exists a vector bundle $N_{X \cong(\Gamma)} X$ over the topological space $X^{\cong}(\Gamma)$ together with a $\Gamma$ action on $N_{X \cong(\Gamma)} X$ such that $N_{X \cong(\Gamma)} X / \Gamma$ is diffeomorphic to a neighborhood of $X \cong(\Gamma)$ in $X$. However such a vector bundle $N_{X \cong(\Gamma)} X$ does not exist in general. In fact, we have the following counter example. 
Example 4.1. We consider $\mathbb{C}^{n} \times S^{1}$ with $\mathbb{Z}_{p}$ action defined by

$$
[k] \cdot(z,[t])=(\exp (2 \pi \sqrt{-1} k / p) z,[t]) .
$$

Here $[k] \in \mathbb{Z} \bmod p,[t] \in \mathbb{R} / \mathbb{Z}=S^{1}$.

We define an isomorphism

$$
F:\left(\mathbb{C}^{n} \times S^{1}\right) / \mathbb{Z}_{p} \rightarrow\left(\mathbb{C}^{n} \times S^{1}\right) / \mathbb{Z}_{p}
$$

by

$$
F([z,[t]])=[\exp (2 \pi \sqrt{-1} t / p) z,[t]] .
$$

We take two copies $\left(\mathbb{C}^{n} \times D_{ \pm}^{2}\right) / \mathbb{Z}_{p}$ of $\left(\mathbb{C}^{n} \times D^{2}\right) / \mathbb{Z}_{p}$ where $\mathbb{Z}_{p}$ action is similar to (13). We identify

$$
\left(\mathbb{C}^{n} \times S^{1}\right) / \mathbb{Z}_{p}=\left(\mathbb{C}^{n} \times \partial D_{+}^{2}\right) / \mathbb{Z}_{p}
$$

with

$$
\left(\mathbb{C}^{n} \times S^{1}\right) / \mathbb{Z}_{p}=\left(\mathbb{C}^{n} \times \partial D_{-}^{2}\right) / \mathbb{Z}_{p}
$$

by $F$ and obtain an orbifold $X$.

In this example $X^{\cong}\left(\mathbb{Z}_{p}\right)=S^{2}$. The normal bundle $N_{X \cong\left(\mathbb{Z}_{p}\right)} X$ does not exist since $F$ does not lift to a bundle isomorphism : $\mathbb{C}^{n} \times S^{1} \rightarrow \mathbb{C}^{n} \times S^{1}$.

As Example 4.1 shows, the normal bundle of the singular locus $X^{\cong}(\Gamma)$ does not exist in general as a global quotient of a vector bundle with a $\Gamma$ action. On the other hand, in this paper we need to use the normal bundle of $X^{\cong}(\Gamma)$ to define normally conical perturbations. For this purpose we define the notion of a normal bundle in the sense of stack. We restrict our discussion of the stack to the case we use for this purpose. Related material is discussed in various references such as [2], [15]. The discussion here is related to the phenomenon that occurs when we remove the effectivity of the $\Gamma_{p}$ action from the definition of orbifold. We feel that the results of Sections 4-6 are not really new. However it is hard to find a reference that contains the results written in a way we want to use. Also most of the references on the stack are written in a very abstract way. To minimize our usage of many abstract languages entering in the definition of stack-like objects, we prefer to use more down-to-earth approach by explicitly writing down all the formulas that we really need. This is the reason why we include the materials in this paper.

Let $G$ be a group. We consider the category $\underline{G}$ which has only one object * and morphism $\underline{G}(*, *)=G$. Let $M$ be a topological space and $\mathcal{U}=\left\{U_{i} \mid i \in I\right\}$ 
be an open covering of $M$. We assume that $U_{i_{1}} \cap \cdots \cap U_{i_{k}}\left(i_{1}, \ldots, i_{k} \in I\right)$ are either empty or contractible. Namely we take a good covering.

Definition 4.2. (i) A sheaf of category of $\underline{G}$ on $(M, \mathcal{U})$ consists of pair $\left(\left\{h_{i j}\right\},\left\{\gamma_{i j k}\right\}\right)$ of an isomorphism

$$
h_{i j} \in \operatorname{Aut}(G) \quad \text { for each } U_{i} \cap U_{j} \neq \emptyset
$$

and an element

$$
\gamma_{i j k} \in G \quad \text { for each } U_{i} \cap U_{j} \cap U_{k} \neq \emptyset
$$

such that the following compatibility conditions (14a) and (14b) hold for every $U_{i} \cap U_{j} \cap U_{k} \cap U_{l} \neq \emptyset$ :

$$
\begin{aligned}
& h_{i j} \circ h_{j k}=\gamma_{i j k} \cdot h_{i k} \cdot \gamma_{i j k}^{-1}, \\
& \gamma_{i j k} \cdot \gamma_{i k l}=h_{i j}\left(\gamma_{j k l}\right) \cdot \gamma_{i j l} .
\end{aligned}
$$

Here the right hand side of $(14 \mathrm{a})$ means $\operatorname{ad}\left(\gamma_{i j k}\right) \circ h_{i k}$. (We will explain how (14) follows from the definition of a stack in the categorical context in Remark $4.5(\mathrm{v})$.)

(ii) $\left(\left\{h_{i j}\right\},\left\{\gamma_{i j k}\right\}\right)$ is said to be isomorphic to $\left(\left\{h_{i j}^{\prime}\right\},\left\{\gamma_{i j k}^{\prime}\right\}\right)$ if there exist

$$
\psi_{i} \in \operatorname{Aut}(G), \quad \mu_{i j} \in G
$$

for each $i$ and $U_{i} \cap U_{j} \neq \emptyset$ respectively, and

$$
h_{i j}^{\prime \prime} \in \operatorname{Aut}(G), \quad \gamma_{i j k}^{\prime \prime} \in G
$$

for each $i, U_{i} \cap U_{j} \neq \emptyset$, and $U_{i} \cap U_{j} \cap U_{k} \neq \emptyset$ respectively such that

$$
\begin{aligned}
h_{i j}^{\prime \prime} & =\psi_{i} \circ h_{i j} \circ \psi_{j}^{-1}, \\
\gamma_{i j k}^{\prime \prime} & =\psi_{i}\left(\gamma_{i j k}\right), \\
\mu_{i j} \cdot h_{i j}^{\prime \prime} \cdot \mu_{i j}^{-1} & =h_{i j}^{\prime}, \\
\mu_{i j} \cdot h_{i j}^{\prime \prime}\left(\mu_{j k}\right) \cdot \gamma_{i j k}^{\prime \prime} & =\gamma_{i j k}^{\prime} \cdot \mu_{i k} .
\end{aligned}
$$

We call a pair $\left(\left\{\mu_{i j}\right\},\left\{\psi_{i}\right\}\right)$ an isomorphism : $\left(\left\{h_{i j}\right\},\left\{\gamma_{i j k}\right\}\right) \rightarrow$ $\left(\left\{h_{i j}^{\prime}\right\},\left\{\gamma_{i j k}^{\prime}\right\}\right)$. We will prove in Lemma 4.2 that we can compose isomorphism and 'isomorphic' defines an equivalence relation.

(iii) We denote by $\operatorname{Sh}((M, \mathcal{U}) ; \underline{G})$ the set of all isomorphism classes of sheaves of categories of $\underline{G}$ on $(M, \mathcal{U})$. 
To illustrate the meaning of (14) we show the following:

Lemma 4.1. Let $\left\{Y_{i}\right\}_{i}$ be a collection of sets such that a group $G$ acts effectively on each of $Y_{i}$. Suppose that there is a point with trivial isotropy group on each of $Y_{i}$. Let $h_{i j}: G \rightarrow G$ be group isomorphisms, $\gamma_{i j k}$ elements of $G$, and let $\phi_{i j}: Y_{j} \rightarrow Y_{i}$ be maps that are injective and $h_{i j}$-equivariant. We assume

$$
\phi_{i j} \circ \phi_{j k}=\gamma_{i j k} \cdot \phi_{i k} \text {. }
$$

Then $\gamma_{i j k}$ satisfies (14).

Proof. Let $g \in G$, and $y \in Y_{k}$ with trivial isotropy group. Then $\phi_{i k}(y)$ also has a trivial isotropy group because $\phi_{i k}$ are assumed to be injective and $h_{i k}$-equivariant.

Again by the $h_{i j}$-equivariance of the map $\phi_{i k}$, we obtain

$$
\begin{aligned}
\gamma_{i j k} \cdot h_{i k}(g) \cdot \phi_{i k}(y) & =\gamma_{i j k} \cdot \phi_{i k}(g \cdot y)=\phi_{i j}\left(\phi_{j k}(g \cdot y)\right) \\
& =h_{i j}\left(h_{j k}(g)\right) \cdot \phi_{i j}\left(\phi_{j k}(y)\right)=h_{i j}\left(h_{j k}(g)\right) \cdot \gamma_{i j k} \cdot \phi_{i k}(y) .
\end{aligned}
$$

Because $\phi_{i k}(y)$ has a trivial isotropy group, (14a) follows.

Similarly for $y \in Y_{l}$ we calculate

$$
\begin{aligned}
h_{i j}\left(\gamma_{j k l}\right) \cdot \gamma_{i j l} \cdot \phi_{i l}(y) & =h_{i j}\left(\gamma_{j k l}\right) \cdot \phi_{i j}\left(\phi_{j l}(y)\right)=\phi_{i j}\left(\gamma_{j k l} \cdot \phi_{j l}(y)\right) \\
& =\phi_{i j}\left(\phi_{j k}\left(\phi_{k l}(y)\right)\right)=\gamma_{i j k} \cdot \phi_{i k}\left(\phi_{k l}(y)\right)=\gamma_{i j k} \cdot \gamma_{i k l} \cdot \phi_{i l}(y) .
\end{aligned}
$$

This implies (14b).

In the definition of Kuranishi structure the group $\Gamma_{p}$ at each point $p$ is assumed to be a finite group and the space $V_{p}$ is a smooth manifold. One can show that effectivity of the action of $\Gamma_{p}$ automatically implies existence of a point with trivial isotropy group. And we also assume that the map $\phi_{p q}$ is an $h_{p q}$-equivariant embedding and in particular injective. Therefore the same argument used in the proof of Lemma 4.1, implies that $\gamma_{p q r}$ in Definition 2.3 satisfies (14b).

Lemma 4.2. The relation 'isomorphism' in Definition 4.2 is an equivalence relation.

Proof. We use notation of (15) and put

$$
\left(h_{i j}^{\prime \prime}, \gamma_{i j k}^{\prime \prime}\right)=\left(1, \psi_{i}\right)_{*}\left(h_{i j}, \gamma_{i j k}\right), \quad\left(h_{i j}^{\prime}, \gamma_{i j k}^{\prime}\right)=\left(\mu_{i j}, 1\right)_{*}\left(h_{i j}^{\prime \prime}, \gamma_{i j k}^{\prime \prime}\right) .
$$

We also put $\left(\mu_{i j}, 1\right)_{*} \circ\left(1, \psi_{i}\right)_{*}=\left(\mu_{i j}, \psi_{i}\right)_{*}$. We note that

$$
\left(1, \psi_{i}\right)_{*} \circ\left(1, \psi_{i}^{\prime}\right)_{*}=\left(1, \psi_{i} \circ \psi_{i}^{\prime}\right)_{*} .
$$


We next claim

$$
\left(\mu_{i j}, 1\right)_{*} \circ\left(\mu_{i j}^{\prime}, 1\right)_{*}=\left(\mu_{i j} \cdot \mu_{i j}^{\prime}, 1\right)_{*} .
$$

Let us prove (18). We put

$$
\left(\mu_{i j}^{\prime}, 1\right)_{*}\left(h_{i j}^{1}, \gamma_{i j k}^{1}\right)=\left(h_{i j}^{2}, \gamma_{i j k}^{2}\right), \quad\left(\mu_{i j}, 1\right)_{*}\left(h_{i j}^{2}, \gamma_{i j k}^{2}\right)=\left(h_{i j}^{3}, \gamma_{i j k}^{3}\right) .
$$

Then

$$
\begin{aligned}
& h_{i j}^{2}=\mu_{i j}^{\prime} \cdot h_{i j}^{1} \cdot\left(\mu_{i j}^{\prime}\right)^{-1}, \\
& \gamma_{i j k}^{2}=\mu_{i j}^{\prime} \cdot h_{i j}^{1}\left(\mu_{j k}^{\prime}\right) \cdot \gamma_{i j k}^{1} \cdot\left(\mu_{i k}^{\prime}\right)^{-1} .
\end{aligned}
$$

Therefore we have

$$
h_{i j}^{3}=\mu_{i j} \cdot \mu_{i j}^{\prime} \cdot h_{i j}^{1} \cdot\left(\mu_{i j}^{\prime}\right)^{-1} \cdot \mu_{i j}^{-1}
$$

and

$$
\begin{aligned}
\gamma_{i j k}^{3} & =\mu_{i j} \cdot h_{i j}^{2}\left(\mu_{j k}\right) \cdot \gamma_{i j k}^{2} \cdot \mu_{i k}^{-1} \\
& =\mu_{i j} \cdot \mu_{i j}^{\prime} \cdot h_{i j}^{1}\left(\mu_{j k}\right) \cdot\left(\mu_{i j}^{\prime}\right)^{-1} \cdot \mu_{i j}^{\prime} \cdot h_{i j}^{1}\left(\mu_{j k}^{\prime}\right) \cdot \gamma_{i j k}^{1} \cdot\left(\mu_{i k}^{\prime}\right)^{-1} \cdot \mu_{i k}^{-1} \\
& =\mu_{i j} \cdot \mu_{i j}^{\prime} \cdot h_{i j}^{1}\left(\mu_{j k} \cdot \mu_{j k}^{\prime}\right) \cdot \gamma_{i j k}^{1} \cdot\left(\mu_{i k} \cdot \mu_{i k}^{\prime}\right)^{-1} .
\end{aligned}
$$

(18) is proved.

We next claim

$$
\left(1, \psi_{i}\right)_{*} \circ\left(\mu_{i j}, 1\right)_{*}=\left(\psi_{i}\left(\mu_{i j}\right), \psi_{i}\right)_{*} .
$$

Let us prove (19). We put

$$
\left(\mu_{i j}, 1\right)_{*}\left(h_{i j}^{1}, \gamma_{i j k}^{1}\right)=\left(h_{i j}^{2}, \gamma_{i j k}^{2}\right), \quad\left(1, \psi_{i}\right)_{*}\left(h_{i j}^{2}, \gamma_{i j k}^{2}\right)=\left(h_{i j}^{3}, \gamma_{i j k}^{3}\right) .
$$

Then

$$
\begin{aligned}
& h_{i j}^{2}=\mu_{i j} \cdot h_{i j}^{1} \cdot \mu_{i j}^{-1}, \\
& \gamma_{i j k}^{2}=\mu_{i j} \cdot h_{i j}^{1}\left(\mu_{j k}\right) \cdot \gamma_{i j k}^{1} \cdot\left(\mu_{i k}\right)^{-1} .
\end{aligned}
$$

Therefore we have

$$
h_{i j}^{3}=\psi_{i} \circ\left(\mu_{i j} \cdot h_{i j}^{1} \cdot \mu_{i j}^{-1}\right) \circ \psi_{j}^{-1}=\psi_{i}\left(\mu_{i j}\right) \cdot\left(\psi_{i} \circ h_{i j}^{1} \circ \psi_{j}^{-1}\right) \cdot \psi_{i}\left(\mu_{i j}\right)^{-1}
$$

and

$$
\gamma_{i j k}^{3}=\psi_{i}\left(\mu_{i j}\right) \cdot \psi_{i}\left(h_{i j}^{1}\left(\mu_{j k}\right)\right) \cdot \psi_{i}\left(\gamma_{i j k}^{1}\right) \cdot \psi_{i}\left(\mu_{i k}\right)^{-1} .
$$

We next put

$$
\left(1, \psi_{i}\right)_{*}\left(h_{i j}^{1}, \gamma_{i j k}^{1}\right)=\left(h_{i j}^{4}, \gamma_{i j k}^{4}\right), \quad\left(\psi_{i}\left(\mu_{i j}\right), 1\right)_{*}\left(h_{i j}^{4}, \gamma_{i j k}^{4}\right)=\left(h_{i j}^{5}, \gamma_{i j k}^{5}\right) .
$$

Then

$$
h_{i j}^{4}=\psi_{i} \circ h_{i j}^{1} \circ \psi_{j}^{-1}, \quad \gamma_{i j k}^{4}=\psi_{i}\left(\gamma_{i j k}^{1}\right) .
$$


Therefore $h_{i j}^{5}=h_{i j}^{3}$ and

$$
\begin{aligned}
\gamma_{i j k}^{5} & =\psi_{i}\left(\mu_{i j}\right) \cdot h_{i j}^{4}\left(\psi_{j}\left(\mu_{j k}\right)\right) \cdot \gamma_{i j k}^{4} \cdot \psi_{i}\left(\mu_{i k}\right)^{-1} \\
& =\psi_{i}\left(\mu_{i j}\right) \cdot \psi_{i}\left(h_{i j}^{1}\left(\mu_{j k}\right)\right) \cdot \psi_{i}\left(\gamma_{i j k}^{1}\right) \cdot \psi_{i}\left(\mu_{i k}\right)^{-1}=\gamma_{i j k}^{3} .
\end{aligned}
$$

(19) is proved.

(17), (18) and (19) imply that we can compose isomorphisms. Hence the relation 'isomorphic' is transitive.

On the other hand, (17) and (18) imply that each isomorphism has an inverse. Hence the relation 'isomorphic' is symmetric.

Remark 4.3. Suppose $\left(h_{i j}, \gamma_{i j k}\right)$ satisfies (14). If we define $\left(h_{i j}^{\prime}, \gamma_{i j k}^{\prime}\right)$ by (15), we can check that $\left(h_{i j}^{\prime}, \gamma_{i j k}^{\prime}\right)$ satisfies $(14)$, in a similar way as the above calculation. For example we consider the case $\psi_{i}=1$ and check (14b) as follows. We have

$$
\begin{aligned}
& \gamma_{i j k}^{\prime} \cdot \gamma_{i k l}^{\prime} \\
& =\mu_{i j} \cdot h_{i j}\left(\mu_{j k}\right) \cdot \gamma_{i j k} \cdot h_{i k}\left(\mu_{k l}\right) \cdot \gamma_{i k l} \cdot \mu_{i l}^{-1} \\
& =\mu_{i j} \cdot h_{i j}\left(\mu_{j k}\right) \cdot \gamma_{i j k} \cdot h_{i k}\left(\mu_{k l}\right) \cdot \gamma_{i j k}^{-1} \cdot h_{i j}\left(\gamma_{j k l}\right) \cdot \gamma_{i j l} \cdot \mu_{i l}^{-1} .
\end{aligned}
$$

On the other hand, we have

$$
\begin{aligned}
& h_{i j}^{\prime}\left(\gamma_{j k l}^{\prime}\right) \cdot \gamma_{i j l}^{\prime} \\
& =\mu_{i j} \cdot h_{i j}\left(\mu_{j k} \cdot h_{j k}\left(\mu_{k l}\right) \cdot \gamma_{j k l} \cdot \mu_{j l}^{-1}\right) \cdot \mu_{i j}^{-1} \cdot \mu_{i j} \cdot h_{i j}\left(\mu_{j l}\right) \cdot \gamma_{i j l} \cdot \mu_{i l}^{-1} \\
& =\mu_{i j} \cdot h_{i j}\left(\mu_{j k}\right) \cdot \gamma_{i j k} \cdot h_{i k}\left(\mu_{k l}\right) \cdot \gamma_{i j k}^{-1} \cdot h_{i j}\left(\gamma_{j k l}\right) \cdot h_{i j}\left(\mu_{j l}^{-1}\right) \cdot \mu_{i j}^{-1} \\
& \quad \cdot \mu_{i j} \cdot h_{i j}\left(\mu_{j l}\right) \cdot \gamma_{i j l} \cdot \mu_{i l}^{-1} .
\end{aligned}
$$

Hence follows (14b).

Definition 4.4. Let $\mathcal{U}^{\prime}=\left\{U_{j}^{\prime} \mid j \in J\right\}$ be another covering of $M$ and let $i(\cdot): j \mapsto i(j)$ be a map $J \rightarrow I$ such that $U_{j}^{\prime} \subseteq U_{i(j)}$. We define a map:

$$
i(\cdot)^{*}: \operatorname{Sh}((M, \mathcal{U}) ; \underline{G}) \rightarrow \operatorname{Sh}\left(\left(M, \mathcal{U}^{\prime}\right) ; \underline{G}\right)
$$

by

$$
i(\cdot)^{*}\left(\left[\left\{h_{i_{1} i_{2}}\right\},\left\{\gamma_{i_{1} i_{2} i_{3}}\right\}\right]\right)=\left[\left\{h_{j_{1} j_{2}}^{\prime}\right\},\left\{\gamma_{j_{1} j_{2} j_{3}}^{\prime}\right\}\right]
$$

where

$$
h_{j_{1} j_{2}}^{\prime}=h_{i\left(j_{1}\right) i\left(j_{2}\right)}, \quad \gamma_{j_{1} j_{2} j_{3}}^{\prime}=\gamma_{i\left(j_{1}\right) i\left(j_{2}\right) i\left(j_{3}\right)} .
$$


We thus obtain an inductive system $\mathcal{U} \mapsto S h((M, \mathcal{U}) ; \underline{G})$. We take the inductive limit with respect to this inductive system and define

$$
\operatorname{Sh}(M, \underline{G})=\underline{\lim } \operatorname{Sh}((M, \mathcal{U}) ; \underline{G}) .
$$

An element of $S h(M, \underline{G})$ is said to be a sheaf of category $\underline{G}$ on $M$.

Remark 4.5. (i) There is a more general notion, that is, a stack in the literature. It was defined by Grothendieck ([17], [18]). See also [2], [15]. We only consider the case we use for our purpose where the stalk of the sheaf is the category $\underline{G}$ which is independent of the point.

(ii) In case when $G$ is commutative, (14a) implies

$$
h_{i j} \circ h_{j k}=h_{i k} .
$$

Therefore it defines a $G$ local system $\mathfrak{G}$. Then (14b) becomes

$$
\gamma_{i j k}+\gamma_{i k l}=h_{i j}\left(\gamma_{j k l}\right)+\gamma_{i j l} .
$$

Namely $\left\{\gamma_{i j k}\right\}$ defines a Čech cocycle in $\check{C}^{2}(\mathcal{U}, \mathfrak{G})$.

Next we assume that $\left(\left\{h_{i j}\right\},\left\{\gamma_{i j k}\right\}\right)$ is isomorphic to $\left(\left\{h_{i j}^{\prime}\right\},\left\{\gamma_{i j k}^{\prime}\right\}\right)$. Then (15a) and (15b) imply that the induced local system is isomorphic and $\left\{\gamma_{i j k}^{\prime \prime}\right\}$ is the same Cech cocycle as $\left\{\gamma_{i j k}\right\}$ under this isomorphism. (15c) and (15d) imply that

$$
\gamma_{i j k}^{\prime}-\gamma_{i j k}^{\prime \prime}=\mu_{i j}+h_{i j}^{\prime \prime}\left(\mu_{j k}\right)-\mu_{i k} .
$$

Namely $\left\{\gamma_{i j k}^{\prime}\right\}$ is cohomologous to $\left\{\gamma_{i j k}^{\prime \prime}\right\}$. Thus

$$
S h(M, \underline{G}) \cong \bigcup_{\mathfrak{G}: G \text { local systems }} \check{H}^{2}(M ; \mathfrak{G})
$$

in the abelian case.

(iii) Usually (but not always) the effectivity of the (finite) group $\Gamma_{p}$ action on $V_{p}$ is assumed when one defines the notion of a chart $\left(V_{p}, \Gamma_{p}, \psi_{p}\right)$ of an orbifold. On the other hand, there is no such assumptions for stacks.

Note (14a) is the same formula as the first formula of Definition 2.3 (ii) in the definition of Kuranishi structure. In Definition 2.3 (ii) we assumed only the existence of $\gamma_{p q r}$. Namely it is not a part of the structure. Also the formula corresponding to (14b) is not in Definition 2.3. On the other hand, in Definition 4.2 we include $\gamma_{i j k}$ as a part of the structure. (Note in Definition 4.2, $\gamma_{i j k}^{\alpha}$ may depend on the connected component $\alpha$. Here $V_{p} \cap V_{q} \cap V_{r}$ is connected since we assume our cover is a good cover.) 
Actually, in the situation of Definition 2.3 where the $\Gamma_{p}$ action is assumed to be effective, the element $\gamma_{p q r}$ satisfying Definition 2.3 (ii) is unique if it exists. Moreover a formula corresponding to (14b) can be proved. (Lemma 4.1.)

In our situation where the $G$ action on $M$ is trivial, $\gamma_{i j k}$ is not determined from the other data and so we include it as a part of the structure. Also (14b) is put as a part of conditions.

When the notion of orbifold was discovered by Satake [22], he assumed the effectivity of the action of $\Gamma_{p}$. Later when Thurston renamed Satake's $\mathrm{V}$-manifold as an orbifold, he did not change its mathematical content and still assumed the effectivity of $\Gamma_{p}$. Because of this, we include the effectivity of $\Gamma_{p}$ as a part of the definition of orbifold in this paper.

(iv) Consider the situation of Definition 2.3. Then, in Lemma 4.1, we proved the equality

$$
\gamma_{p q r} \cdot \gamma_{p r s}=h_{p q}\left(\gamma_{q r s}\right) \cdot \gamma_{p q s}
$$

where $q \in \psi_{p}\left(s_{p}^{-1}(0) / \Gamma_{p}\right), r \in \psi_{q}\left(s_{q}^{-1}(0) / \Gamma_{q}\right), s \in \psi_{r}\left(s_{r}^{-1}(0) / \Gamma_{r}\right)$. Since (20) is automatic, we did not put it as a part of assumptions in Definition 2.3. In the situation where effectivity of the $\Gamma_{p}$ action is not assumed, (20) will not be automatic.

(v) Using the language of category theory, we can rewrite the definition of $S h(M, \underline{G})$, as follows. (Our discussion below is informal since we do not use it in this paper.)

We first define a category $\mathcal{O}(M)$. Its objects are open sets of $M$. There is no morphism from $U$ to $V$ if $U$ is not a subset of $V$. If $U \subset V$, there exists exactly one morphism from $U$ to $V$.

We next consider the 2-category $\underline{\underline{G}}$ as follows. There is only one object in it. The category of morphism from this object to itself is $\underline{G}$.

Then an element of $S h(M, \underline{G})$ is regarded as a pseudo-functor from $\mathcal{O}(M)$ to $\underline{\underline{G}}$, in the sense of [18] Exposé VI 8 .

Let us explain how a pseudo-functor $\mathcal{O}(M) \rightarrow \underline{\underline{G}}$ is related to an element of $S h(X, \underline{G})$. A pseudo-functor $\mathcal{O}(M) \rightarrow \underline{\underline{G}}$ first assigns a functor $F_{U V}$ : $\underline{G} \rightarrow \underline{G}$ for each $U \subset V$. Such a functor is nothing but a homomorphism $\phi_{U V}: G \rightarrow G$. 
If $U_{3} \subset U_{2} \subset U_{1}$, then the pseudo-functor associate a natural transformation

$$
T_{U_{3} U_{2} U_{1}}: F_{U_{3} U_{1}} \rightarrow F_{U_{3} U_{2}} \circ F_{U_{2} U_{1}}
$$

which is (in our situation) automatically an equivalence. By definition of the category $\underline{G}$, such a natural transformation is given by an element $\gamma_{U_{3} U_{2} U_{1}} \in$ $G$ such that

$$
\gamma_{U_{3} U_{2} U_{1}} \cdot \phi_{U_{3} U_{1}}=\phi_{U_{3} U_{2}} \circ \phi_{U_{2} U_{1}} .
$$

This formula corresponds to (14a).

For the pair $\left(F_{U V}, \gamma_{U_{3} U_{2} U_{1}}\right)$ to be a pseudo-functor we need to assume a compatibility condition between them, that is the commutativity of the following diagram for each $U_{4} \subset U_{3} \subset U_{2} \subset U_{1}$.

$$
\begin{array}{cccc}
F_{U_{4} U_{3}} \circ F_{U_{3} U_{1}} \stackrel{T_{U_{4} U_{3} U_{1}}}{\longleftarrow} & F_{U_{4} U_{1}} & \stackrel{T_{U_{4} U_{2} U_{1}}}{\longrightarrow} & F_{U_{4} U_{2}} \circ F_{U_{2} U_{1}} \\
\left.\downarrow F_{U_{4} U_{3}}\right)_{*}\left(T_{U_{3} U_{2} U_{1}}\right) & & & \left(F_{U_{2} U_{1}}\right)^{*}\left(T_{U_{4} U_{3} U_{2}}\right) \downarrow \\
F_{U_{4} U_{3}} \circ F_{U_{3} U_{2}} \circ F_{U_{2} U_{1}} & & F_{U_{4} U_{3}} \circ F_{U_{3} U_{2}} \circ F_{U_{2} U_{1}} .
\end{array}
$$

(See Definition 3.10 (iv)(b) [4] or [18] Exposé VI Proposition 7.4.) The commutativity of this diagram is equivalent to

$$
\gamma_{U_{4} U_{3} U_{2}} \cdot \gamma_{U_{4} U_{2} U_{1}}=\phi_{U_{4} U_{3}}\left(\gamma_{U_{3} U_{2} U_{1}}\right) \cdot \gamma_{U_{4} U_{3} U_{1}}
$$

This formula is the same as (14b). (14a) is a consequence of the fact that $T_{U_{3} U_{2} U_{1}}$ is a natural transformation.

We note that, in the definition of pseudo-functor in Definition 3.10 [4], there are other conditions (ii), (iv)(a). In our situation, it will become

$$
h_{i i}(g)=\gamma_{i i i} \cdot g \cdot \gamma_{i i i}^{-1}, \quad \gamma_{i i j}=\gamma_{i i i}, \quad \gamma_{i j j}=h_{i j}\left(\gamma_{j j j}\right) .
$$

They follow from (14). In fact, the first formula follows from (14a) by putting $i=j=k$. The second formula follows from (14b) by putting $i=j=k$. The third formula follows from (14b) by putting $j=k=l$.

We can continue and rewrite (15) using category theory. We do not try to do it here. In fact, the theory of stack which is well established is based on category theory, and in this subsection we try to give a self-contained account of the part thereof which we need, without using category theory.

(vi) We did not assume

$$
h_{i i}=i d, \quad h_{i j} \circ h_{j i}=i d,
$$


in Definition 4.2. There seems to be the version that assumes the above identities together with

$$
\gamma_{i j k}=\gamma_{i k j}^{-1}, \quad \gamma_{i j k}=h_{i j}\left(\gamma_{j k i}\right)
$$

In the abelian case, these conditions will become the condition that $h_{i j \cdots i_{k} k}$ is anti-symmetric with respect to the change of indices. It is well known that we have the same Cech cohomology, whether or not we assume the anti-symmetricity.

\section{The Stack structure of the Singular locus}

Now we apply the above discussion of stacks to the circumstance that arises in later sections in relation to the study of normal bundles of the singular locus $X^{\cong}(\Gamma)$.

Example-Definition 5.1. Let $G$ be a finite group acting effectively and smoothly on a smooth manifold $\tilde{X}$. Consider the orbifold $X=\tilde{X} / G$. Such an orbifold is said to be a global quotient. Let $\Gamma$ be an abstract group. We put

$$
X^{\cong}(\Gamma)=\left\{x \in \widetilde{X} \mid I_{x} \cong \Gamma\right\} / G,
$$

where

$$
I_{x}=\{g \in G \mid g x=x\} .
$$

It follows from (21) below that $X^{\cong}(\Gamma)$ is a smooth manifold.

We now give a construction of an element of $S h\left(X^{\cong}(\Gamma), \underline{G}\right)$. We decompose $X^{\cong}(\Gamma)$ as in (21) and study each of them separately. Namely, for each subgroup $\Gamma_{0} \subset G$ with $\Gamma_{0} \cong \Gamma$, we consider

$$
\widetilde{X}^{=}\left(\Gamma_{0}\right)=\left\{x \in \widetilde{X} \mid I_{x}=\Gamma_{0}\right\}
$$

Denote the normalizer of $\Gamma_{0}$ by

$$
N\left(\Gamma_{0}\right)=\left\{g \in G \mid g \Gamma_{0} g^{-1}=\Gamma_{0}\right\} .
$$

Then $H\left(\Gamma_{0}\right)=N\left(\Gamma_{0}\right) / \Gamma_{0}$ acts freely on $\widetilde{X}^{=}\left(\Gamma_{0}\right)$ and by definition we have

$$
X^{\cong}(\Gamma)=\bigcup_{\Gamma_{0}} \widetilde{X}^{=}\left(\Gamma_{0}\right) / H\left(\Gamma_{0}\right),
$$


where the union is taken over a complete system of representatives of the conjugacy classes of the subgroups of $G$ isomorphic to $\Gamma$. We have an exact sequence

$$
1 \longrightarrow \Gamma_{0} \longrightarrow N\left(\Gamma_{0}\right) \longrightarrow H\left(\Gamma_{0}\right) \longrightarrow 1 \text {. }
$$

We choose a sufficiently fine good covering $\mathcal{U}=\left\{U_{i} \mid i \in I\right\}$ of $X^{\cong}(\Gamma)$ and a lift $\widetilde{U}_{i} \subset \widetilde{X}^{=}\left(\Gamma_{0}\right)$ of $U_{i}$ so that the projection $\widetilde{X} \rightarrow X$ restricts to a homeomorphism from $\widetilde{U}_{i}$ to $U_{i}$.

For each $i, j$ with $U_{i} \cap U_{j} \neq \emptyset$ there exists a unique $\underline{h}_{i j} \in H\left(\Gamma_{0}\right)$ such that $\widetilde{U}_{i} \cap\left(\underline{h}_{i j} \widetilde{U}_{j}\right) \neq \emptyset$. We remark that $\underline{h}_{i j} \cdot \underline{h}_{j k}=\underline{h}_{i k}$ in $H\left(\Gamma_{0}\right)$, if $U_{i} \cap U_{j} \cap U_{k} \neq \emptyset$.

We choose lifts $\underline{\tilde{h}}_{i j} \in N\left(\Gamma_{0}\right)$ of $\underline{h}_{i j}$.

We define an automorphism $h_{i j}: \Gamma_{0} \rightarrow \Gamma_{0}$ by

$$
h_{i j}(g)=\underline{\widetilde{h}}_{i j} \cdot g \cdot \underline{\widetilde{h}}_{i j}^{-1} .
$$

We define $\gamma_{i j k} \in \Gamma_{0}$ by

$$
\gamma_{i j k} \cdot \underline{\tilde{h}}_{i k}=\underline{\tilde{h}}_{i j} \cdot \underline{\tilde{h}}_{j k} .
$$

Then it is easy to check that $\left\{\gamma_{i j k}\right\}$ satisfies (14).

We can generalize the above construction and include the case of an orbifold that is not necessarily a global quotient of a manifold. We do not discuss it here since we do not use it in the main application (the proof of Theorem 3.1).

We note that we can choose $\gamma_{i j k}=1$ if the exact sequence (22) splits. But this is not always the case.

Example 5.2. Let us consider the orbifold $X$ given in Example 4.1. Then $X^{\cong}(\Gamma)=S^{2}$. The $\mathbb{Z}_{p}$ local system is necessarily trivial on $S^{2}$. So

$$
S h\left(S^{2} ; \mathbb{Z}_{p}\right) \cong \check{H}^{2}\left(S^{2} ; \mathbb{Z}_{p}\right) \cong \mathbb{Z}_{p}
$$

The element thereof defined in Example-Definition 5.1 is the generator of $\mathbb{Z}_{p}$ and hence is nonzero.

Lemma 5.1. The element of $S h\left(X^{\cong}(\Gamma), \underline{G}\right)$ represented by $\left(\left\{h_{i j}\right\},\left\{\gamma_{i j k}\right\}\right)$ is independent of various choices involved in the construction.

Proof. We first fix $\mathcal{U}=\left\{U_{i} \mid i \in I\right\}$. We change $\widetilde{U}_{i}$ to $\alpha_{i} \widetilde{U}_{i}$ where $\alpha_{i} \in N\left(\Gamma_{0}\right)$. We also change $\underline{\widetilde{h}}_{i j}$ to

$$
\underline{\tilde{h}}_{i j}^{\prime \prime}=\alpha_{i} \cdot \underline{\tilde{h}}_{i j} \cdot \alpha_{j}^{-1} .
$$


Then $h_{i j} \in \operatorname{Aut}(G)$ is transformed to

$$
h_{i j}^{\prime \prime}=\operatorname{ad}\left(\alpha_{i}\right) \circ h_{i j} \circ \operatorname{ad}\left(\alpha_{j}\right)^{-1},
$$

where ad $: N\left(\Gamma_{0}\right) \rightarrow \operatorname{Aut}\left(\Gamma_{0}\right)$ is defined by $\operatorname{ad}(g)\left(g^{\prime}\right)=g \cdot g^{\prime} \cdot g^{-1}$. We put $\psi_{i}=\operatorname{ad}\left(\alpha_{i}\right)$ and $\mu_{i j}=1$. Then (15a), (15c) are satisfied and hence $\left(\left\{\mu_{i j}\right\},\left\{\psi_{i}\right\}\right)$ defines an isomorphism.

We also have

$$
\gamma_{i j k}^{\prime \prime}=\alpha_{i} \cdot \gamma_{i j k} \cdot \alpha_{i}^{-1}=\psi_{i}\left(\gamma_{i j k}\right) .
$$

Therefore the isomorphism class is independent of the choice of $\widetilde{U}_{i}$.

We next fix $\widetilde{U}_{i}$ and change the lift $\underline{\tilde{h}}_{i j} \in N\left(\Gamma_{0}\right)$ of $\underline{h}_{i j}$. We put

$$
\underline{\tilde{h}}_{i j}^{\prime}=\mu_{i j} \cdot \underline{\tilde{h}}_{i j}
$$

Then we have

$$
\gamma_{i j k}^{\prime}=\underline{\tilde{h}}_{i j}^{\prime} \cdot \underline{\tilde{h}}_{j k}^{\prime} \cdot\left(\underline{\tilde{h}}_{i k}^{\prime}\right)^{-1}=\mu_{i j} \cdot h_{i j}\left(\mu_{j k}\right) \cdot \gamma_{i j k} \cdot \mu_{i k}^{-1}
$$

as required. The invariance under the refinement of the covering is easy to prove.

Definition 5.3. We call the structure defined by $\left(\left\{h_{i j}\right\},\left\{\gamma_{i j k}\right\}\right) \in S h\left(X^{\cong}(\Gamma), \underline{G}\right)$ in Example-Definition 5.1, the standard stack structure on $X^{\cong}(\Gamma)$.

6. The normal Bundle of the Singular locus and $C^{\infty}$ LOCAL TRIVIALITY

Going back to the general topological space $M$, we next define a vector bundle on the stack defined by an element of $S h(M, \underline{G})$. Let $\left(\left\{h_{i j}\right\},\left\{\gamma_{i j k}\right\}\right) \in$ $\operatorname{Sh}((M, \mathcal{U}), \underline{G})$.

Definition 6.1. A vector bundle on $\left(\left\{h_{i j}\right\},\left\{\gamma_{i j k}\right\}\right) \in S h((M, \mathcal{U}), \underline{G})$ is a pair $\left(\left\{F_{i}\right\},\left\{g_{i j}\right\}\right)$ such that $F_{i}$ is a vector bundle on $U_{i}$ with $G$ action and $g_{i j}$ is an $h_{i j}$-equivariant bundle isomorphism $g_{i j}:\left.\left.F_{j}\right|_{U_{i} \cap U_{j}} \rightarrow F_{i}\right|_{U_{i} \cap U_{j}}$ such that:

$$
g_{i j} \circ g_{j k}=\gamma_{i j k} \cdot g_{i k} \text {. }
$$

We assume that $\left(\left\{\mu_{i j}\right\},\left\{\psi_{i}\right\}\right)$ is an isomorphism $\left(\left\{h_{i j}\right\},\left\{\gamma_{i j k}\right\}\right) \rightarrow$ $\left(\left\{h_{i j}^{\prime}\right\},\left\{\gamma_{i j k}^{\prime}\right\}\right)$. An isomorphism from a vector bundle $\mathcal{F}=\left(\left\{F_{i}\right\},\left\{g_{i j}\right\}\right)$ on 
$\left(\left\{h_{i j}\right\},\left\{\gamma_{i j k}\right\}\right)$ to a vector bundle $\mathcal{F}^{\prime}=\left(\left\{F_{i}^{\prime}\right\},\left\{g_{i j}^{\prime}\right\}\right)$ on $\left(\left\{h_{i j}^{\prime}\right\},\left\{\gamma_{i j k}^{\prime}\right\}\right)$ is a family $\left\{\phi_{i}\right\}_{i \in I}$ of $\psi_{i}$-equivariant isomorphisms of vector bundles $\phi_{i}: F_{i} \rightarrow F_{i}^{\prime}$ such that

$$
g_{i j}^{\prime} \circ \phi_{j}=\mu_{i j} \cdot\left(\phi_{i} \circ g_{i j}\right) .
$$

We say $\left\{\phi_{i}\right\}_{i \in I}$ is an isomorphism $: \mathcal{F} \rightarrow \mathcal{F}^{\prime}$ over $\left(\left\{\mu_{i j}\right\},\left\{\psi_{i}\right\}\right)$.

Lemma 6.1. The relation 'isomorphic' in Definition 6.1 is an equivalence relation.

Proof. Let $\left(\mu_{i j}, \psi_{i}\right)_{*}\left(h_{i j}^{1}, \gamma_{i j k}^{1}\right)=\left(h_{i j}^{2}, \gamma_{i j k}^{2}\right)$ and $\left(\mu_{i j}^{\prime}, \psi_{i}^{\prime}\right)_{*}\left(h_{i j}^{2}, \gamma_{i j k}^{2}\right)=\left(h_{i j}^{3}, \gamma_{i j k}^{3}\right)$. Let $\mathcal{F}^{c}=\left(\left\{F_{i}^{c}\right\},\left\{g_{i j}^{c}\right\}\right)$ be a vector bundle on $\left(\left\{h_{i j}^{c}\right\},\left\{\gamma_{i j k}^{c}\right\}\right)$ and $\left\{\phi_{i}\right\}_{i \in I}: \mathcal{F}^{1} \rightarrow$ $\mathcal{F}^{2}$ and $\left\{\phi_{i}^{\prime}\right\}_{i \in I}: \mathcal{F}^{2} \rightarrow \mathcal{F}^{3}$ be isomorphisms over $\left(\left\{\mu_{i j}\right\},\left\{\psi_{i}\right\}\right),\left(\left\{\mu_{i j}^{\prime}\right\},\left\{\psi_{i}^{\prime}\right\}\right)$, respectively. Then we can check easily by calculation that $\left\{\phi_{i}^{\prime} \circ \phi_{i}\right\}_{i \in I}$ is an isomorphism : $\mathcal{F}^{1} \rightarrow \mathcal{F}^{3}$ over $\left(\left\{\mu_{i j}^{\prime}\right\},\left\{\psi_{i}^{\prime}\right\}\right) \circ\left(\left\{\mu_{i j}\right\},\left\{\psi_{i}\right\}\right)=\left(\left\{\mu_{i j}^{\prime} \cdot \psi_{i}^{\prime}\left(\mu_{i j}\right)\right\},\left\{\psi_{i}^{\prime} \circ\right.\right.$ $\left.\left.\psi_{i}\right\}\right)$.

Lemma 6.2. Let $\mathcal{F}=\left(\left\{F_{i}\right\},\left\{g_{i j}\right\}\right)$ be a vector bundle on $\left(\left\{h_{i j}\right\},\left\{\gamma_{i j k}\right\}\right)$ and let $\left(\left\{\mu_{i j}\right\},\left\{\psi_{i}\right\}\right)$ be an isomorphism $\left(\left\{h_{i j}\right\},\left\{\gamma_{i j k}\right\}\right) \rightarrow\left(\left\{h_{i j}^{\prime}\right\},\left\{\gamma_{i j k}^{\prime}\right\}\right)$. Let $F_{i}^{\prime}$ be a $G$ equivariant vector bundle on $U_{i}$ and let $\phi_{i}: F_{i} \rightarrow F_{i}^{\prime}$ be a $\psi_{i}$-equivariant bundle isomorphism. We define $g_{i j}^{\prime}$ by $(24)$. Then $\left(\left\{F_{i}^{\prime}\right\},\left\{g_{i j}^{\prime}\right\}\right)$ is a vector bundle on $\left(\left\{h_{i j}^{\prime}\right\},\left\{\gamma_{i j k}^{\prime}\right\}\right)$.

Proof. It is easy to see that $g_{i j}^{\prime}$ is $h_{i j}^{\prime}$ equivariant. So it suffices to check (23) for $g_{i j}^{\prime}$ and $\gamma_{i j k}^{\prime}$. We may divide the cases into $\left(\mu_{i j}, \psi_{i}\right)=\left(1, \psi_{i}\right)$ and $\left(\mu_{i j}, \psi_{i}\right)=\left(\mu_{i j}, 1\right)$.

In case $\left(\mu_{i j}, \psi_{i}\right)=\left(1, \psi_{i}\right)$ we have

$$
\begin{aligned}
g_{i j}^{\prime} \circ g_{j k}^{\prime} \circ \phi_{k} & =\phi_{i} \circ g_{i j} \circ g_{j k}=\phi_{i}\left(\gamma_{i j k} \cdot g_{i k}\right) \\
& =\psi_{i}\left(\gamma_{i j k}\right) \cdot \phi_{i} \circ g_{i k}=\gamma_{i j k}^{\prime} \cdot g_{i k}^{\prime} \circ \phi_{k}
\end{aligned}
$$

as required.

In case $\left(\mu_{i j}, \psi_{i}\right)=\left(\mu_{i j}, 1\right)$ we have

$$
\begin{aligned}
g_{i j}^{\prime} \circ g_{j k}^{\prime} \circ \phi_{k} & =g_{i j}^{\prime} \circ\left(\mu_{j k} \cdot\left(\phi_{j} \circ g_{j k}\right)\right) \\
& =h_{i j}^{\prime}\left(\mu_{j k}\right) \cdot \mu_{i j} \cdot \phi_{i} \circ\left(g_{i j} \circ g_{j k}\right) \\
& =\mu_{i j} \cdot h_{i j}\left(\mu_{j k}\right) \cdot \gamma_{i j k} \cdot \mu_{i k}^{-1} \cdot\left(g_{i k}^{\prime} \circ \phi_{k}\right) \\
& =\gamma_{i j k}^{\prime} \cdot\left(g_{i k}^{\prime} \circ \phi_{k}\right),
\end{aligned}
$$

as required. The proof of the lemma is now complete. 
We next discuss how a vector bundle behaves under the refinement of the covering. Let

$$
i(\cdot)^{*}\left(\left[\left\{h_{i_{1} i_{2}}\right\},\left\{\gamma_{i_{1} i_{2} i_{3}}\right\}\right]\right)=\left[\left\{h_{j_{1} j_{2}}^{\prime}\right\},\left\{\gamma_{j_{1} j_{2} j_{3}}^{\prime}\right\}\right] .
$$

See Definition 4.4. Then a vector bundle $\left(\left\{F_{i}\right\},\left\{g_{i_{1} i_{2}}\right\}\right)$ on $\left(\left\{h_{i_{1} i_{2}}\right\},\left\{\gamma_{i_{1} i_{2} i_{3}}\right\}\right)$ induces $\left(\left\{F_{j}^{\prime}\right\},\left\{g_{j_{1} j_{2}}^{\prime}\right\}\right)$ on $\left(\left\{h_{j_{1} j_{2}}^{\prime}\right\},\left\{\gamma_{j_{1} j_{2} j_{3}}^{\prime}\right\}\right)$ by

$$
g_{j_{1} j_{2}}^{\prime}=\left.g_{i\left(j_{1}\right) i\left(j_{2}\right)}\right|_{U_{j_{1} j_{2}}^{\prime}} \text {. }
$$

Therefore we can define the notion of a vector bundle on a pair $\left(M,\left[\left\{h_{i j}\right\},\left\{\gamma_{i j k}\right\}\right]\right)$ where $\left[\left\{h_{i j}\right\},\left\{\gamma_{i j k}\right\}\right] \in S h(M, \underline{G})$.

We consider the situation of Example-Definition 5.1 and use the notations there.

Definition 6.2. We define the normal bundle $N_{X \cong(\Gamma)} X$ over $X^{\cong}(\Gamma)$ with the standard stack structure as follows: We identify $U_{i} \subseteq X^{\cong}(\Gamma)$ with $\widetilde{U}_{i} \subset \widetilde{X}^{=}\left(\Gamma_{0}\right)$ by the projection and put $F_{i}=N_{\widetilde{U}_{i}} \tilde{X}$, the normal bundle of $\widetilde{U}_{i}$ in $\tilde{X}$. The $\Gamma_{0}$ $(\subset G)$ action on $\widetilde{X}$ induces one on $F_{i}$.

We next define $g_{i j}$. We have

$$
\left(\underline{\widetilde{h}}_{i j}^{-1} \widetilde{U}_{i}\right) \cap \widetilde{U}_{j} \subset \widetilde{U}_{j} \cong U_{j}
$$

We identify $\left(\widetilde{\underline{h}}_{i j}^{-1}\right) \widetilde{U}_{i} \cap \widetilde{U}_{j}$ with $U_{i} \cap U_{j}$. Then an open embedding

$$
\underline{\underline{h}}_{i j}: U_{i} \cap U_{j} \rightarrow \widetilde{U}_{i} \cong U_{i}
$$

is induced. It extends to a map $\widetilde{X} \rightarrow \widetilde{X}$. Then we have

$$
g_{i j}:=\left(\underline{\widetilde{h}}_{i j} \cdot\right)_{*}:\left.F_{j}\right|_{U_{i} \cap U_{j}}=N_{\widetilde{\underline{h}}_{i j}^{-1} \widetilde{U}_{i}} \widetilde{X} \rightarrow F_{i}=N_{\widetilde{U}_{i}} \widetilde{X} .
$$

Since $\underline{\widetilde{h}}_{i j} \cdot \underline{\widetilde{h}}_{j k}=\gamma_{i j k} \cdot \underline{\widetilde{h}}_{i k}$, we obtain $(23)$. Therefore $\left(\left\{F_{i}\right\},\left\{g_{i j}\right\}\right)$ is a vector bundle on $\left(\left\{h_{i j}\right\},\left\{\gamma_{i j k}\right\}\right) \in S h\left(X^{\cong}(\Gamma), \underline{G}\right)$ in the sense of Definition 6.1. We put

$$
N_{X \cong(\Gamma)} X=\left(\left\{F_{i}\right\},\left\{g_{i j}\right\}\right)
$$

which we call the normal bundle of $X^{\cong}(\Gamma)$.

We can generalize this construction to the case when $X$ is an orbifold, not necessarily a global quotient of a manifold. We do not discuss this here since it is not needed in our main application (the proof of Theorem 3.1).

Lemma 6.3. The vector bundle $\left(\left\{F_{i}\right\},\left\{g_{i j}\right\}\right)$ in Definition 6.2 is independent of the choices involved in the construction up to isomorphism. 
Proof. We use the notation of the proof of Lemma 5.1. Let $\alpha_{i} \in N\left(\Gamma_{0}\right)$.

We first change $\widetilde{U}_{i}$ to $\alpha_{i} \cdot \widetilde{U}_{i}$. Then $\alpha_{i}$ induces a map

$$
\alpha_{i} \cdot N_{\widetilde{U}_{i}} \widetilde{X} \rightarrow N_{\alpha_{i} \cdot \widetilde{U}_{i}} \tilde{X}
$$

It induces

$$
\phi_{i}=\left(\alpha_{i} \cdot\right)_{*}: F_{i} \rightarrow F_{i}^{\prime} .
$$

Since $\psi_{i}=\operatorname{ad}\left(\alpha_{i}\right)$, it follows that $\phi_{i}$ is $\psi_{i}$ equivariant. The equality

$$
\underline{\underline{\underline{h}}}_{i j}^{\prime}=\alpha_{i} \cdot \underline{\underline{h}}_{i j} \cdot \alpha_{j}^{-1}
$$

implies that

$$
g_{i j}^{\prime} \circ \phi_{j}=\phi_{i} \circ g_{i j}
$$

Since $\mu_{i j}=1$ in this case, we obtain the required isomorphism.

We next fix $\left\{\widetilde{U}_{i}\right\}$ and change the lift $\left\{\underline{\widetilde{h}}_{i j}\right\}$ to

$$
\underline{\underline{\underline{h}}}_{i j}^{\prime}=\mu_{i j} \cdot \underline{\tilde{h}}_{i j} \text {. }
$$

In this case we have $F_{i}=F_{i}^{\prime}$ and $\phi_{i}=$ identity. (25) implies

$$
g_{i j}^{\prime}=\left(\widetilde{\widetilde{h}}_{i j}^{\prime}\right)_{*}=\mu_{i j} \cdot\left(\underline{\widetilde{\underline{h}}}_{i j}\right)_{*}=\mu_{i j} \cdot g_{i j} .
$$

The invariance under the refinement of the covering is easy to prove.

Example 6.3. In the situation of Example 4.1, the normal bundle does not exist as a (usual) vector bundle on $S^{2}$. But it exists over $S^{2}$ with nontrivial stack structure, which corresponds to the generator of $\check{H}^{2}\left(S^{2} ; \mathbb{Z}_{p}\right)$. See Example 5.2.

Definition 6.4. We consider the situation of Example-Definition 5.1 and let $\widetilde{E}$ be a $G$ equivariant vector bundle on $\widetilde{X}$. It induces an orbi-bundle $E$ on $X=\widetilde{X} / G$. We define a vector bundle $\left.E\right|_{X \cong(\Gamma)}$ on $X^{\cong}(\Gamma)$ as follows: We use the notation in Example-Definition 5.1 and Definition 6.2.

We put

$$
E_{i}=\left.\widetilde{E}\right|_{\widetilde{U}_{i}}
$$

and regard it as a vector bundle on $U_{i}$. As in Definition 6.2, we have an open embedding

$$
\underline{\underline{h}}_{i j}: U_{i} \cap U_{j} \rightarrow \widetilde{U}_{i} \cong U_{i} .
$$

Since $\underline{\underline{h}}_{i j} \in G$, it induces a bundle map

$$
g_{i j}:\left.E_{j}\right|_{U_{i} \cap U_{j}} \rightarrow E_{i} .
$$


It follows that $g_{i j}$ satisfies the required relation in the same way as in Definition 6.2. We define

$$
\left.E\right|_{X \cong(\Gamma)}=\left(\left\{E_{i}\right\},\left\{g_{i j}\right\}\right)
$$

and call it the restriction of $E$ to $X^{\cong}(\Gamma)$.

We can also generalize this construction to the case of an orbifold which is not necessarily a global quotient.

In the same way as Lemma 6.3, we can prove that $\left(\left\{E_{i}\right\},\left\{g_{i j}\right\}\right)$ is independent of the choices up to isomorphism.

Before proceeding further, we review the definitions of diffeomorphisms between orbifolds and of locally trivial $C^{\infty}$ fiber bundles with orbifolds as fibers. Those notions will be used in Sections 7, 8 and 9. They are of course well established. However there are several delicate points in its definition. Since those points are related to the arguments of later sections, we state a precise definition that we use in this paper.

Let $\tilde{X}$ be a smooth manifold and $G$ a finite group acting effectively on it. The quotient $X=\widetilde{X} / G$ defines an orbifold. Let $X^{\prime}=\widetilde{X}^{\prime} / G^{\prime}$ be another global quotient and $W \subset X, W^{\prime} \subset X^{\prime}$ open subsets.

Definition 6.5. A homeomorphism $F: W \rightarrow W^{\prime}$ is said to be a diffeomorphism if the following holds for every $p \in W$ and $p^{\prime}=F(p)$.

Let $\tilde{p} \in \tilde{X}, \tilde{p}^{\prime} \in \tilde{X}^{\prime}$ be lifts of $p$ and $p^{\prime}$, respectively. Then there exist open neighborhoods $\widetilde{U}, \widetilde{U}^{\prime}$ of them, an isomorphism $\phi: I_{\tilde{p}} \rightarrow I_{\tilde{p}^{\prime}}$ and a $\phi$-equivariant diffeomorphism $\tilde{F}_{p}: \widetilde{U} \rightarrow \widetilde{U}^{\prime}$ such that $\widetilde{U} / I_{\tilde{p}}, \widetilde{U}^{\prime} / I_{\tilde{p}^{\prime}}$ are open subsets of $X, X^{\prime}$ and that $\widetilde{F}$ induces the restriction of the map $F$ to $\widetilde{U} / I_{\tilde{p}}$.

Definition 6.6. Let $N$ be a smooth manifold, $X=\widetilde{X} / G, X^{\prime}=\tilde{X}^{\prime} / G^{\prime}$ global quotients, and $W \subset X, F \subset X^{\prime}$ open subsets. A continuous map $\pi: W \rightarrow N$ is said to be a locally trivial fiber bundle of $C^{\infty}$-class with fiber $F$ if the following holds.

For each $p \in N$ there exist an open neighborhood $U_{p}$ of it and a diffeomorphism $\phi_{p}: F \times U_{p} \rightarrow \pi^{-1}\left(U_{p}\right)$ in the sense of Definition 6.5, such that the following 
diagram commutes.

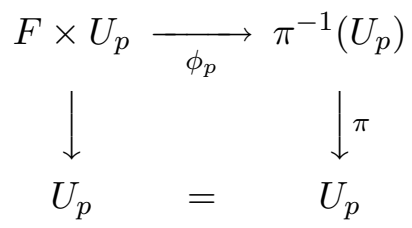

Remark 6.7. We require the commutativity of the Diagram (26) set theoretically. In general, the notion of morphisms between orbifolds must be carefully defined. In fact, the systematic study of it requires to use the notion of 2 category. In this paper, we assume effectivity of the finite group action in the definition of orbifold and in Definition 6.6 we assumed the base space $N$ is a manifold. So it suffices to require the commutativity of Diagram (26) set theoretically, in our situation.

Now let $\mathcal{F}=\left(\left\{F_{i}\right\},\left\{g_{i j}\right\}\right)$ be a vector bundle over $\left(\left\{h_{i j}\right\},\left\{\gamma_{i j k}\right\}\right) \in \operatorname{Sh}(M, \underline{G})$. Assume that the $G$ action on the fibers of $F_{i}$ is effective. We are going to define an orbifold structure on $\mathcal{F} / G$. We define an equivalence relation $\sim$ on $\bigcup_{i} F_{i}$ as follows. (Here $F_{i}$ also denotes the total space of the vector bundle $F_{i}$ over $U_{i}$.) Let $x \in F_{i}$ and $y \in F_{j}$. Then $x \sim y$ if and only if one of the following holds.

(1) $i=j, x=\gamma y$ for some $\gamma \in G$.

(2) $\pi(x) \in U_{i} \cap U_{j}, y=\gamma \cdot g_{j i}(x)$ for some $\gamma \in G$. Here $\pi: F_{i} \rightarrow U_{i}$, $\pi: F_{j} \rightarrow U_{j}$ are the projections.

It is easy to see that $\sim$ is an equivalence relation. We put

$$
|\mathcal{F} / G|=\bigcup_{i} F_{i} / \sim
$$

and define a quotient topology on it. The projection $\pi: F_{i} \rightarrow U_{i} \subset M$ induces a map

$$
\pi:|\mathcal{F} / G| \rightarrow M
$$

Let $F$ be the fiber of the vector bundle $F_{i} . F$ is a vector space on which $G$ acts effectively. We assume that $M$ is a smooth manifold.

Lemma 6.4. $|\mathcal{F} / G|$ has a structure of an orbifold. We denote it by $\mathcal{F} / G$. If $\mathcal{F}$ is isomorphic to $\mathcal{F}^{\prime}$, then $\mathcal{F} / G$ is diffeomorphic to $\mathcal{F}^{\prime} / G$ as an orbifold.

Moreover, $\pi: \mathcal{F} / G \rightarrow M$ is a locally trivial fiber bundle of $C^{\infty}$-class, whose fiber is $F / G$. 
Proof. Let $U_{i_{1} \ldots i_{k}}=\bigcap_{j=1}^{k} U_{i_{j}}$. The bundle isomorphism $g_{i j}$ induces an $h_{i j}$ equivariant embedding $\left.F_{j}\right|_{U_{i j}} \rightarrow F_{i}$, which we also denote by $g_{i j}$. By the definition of a vector bundle over $\left(\left\{h_{i j}\right\},\left\{\gamma_{i j k}\right\}\right)$, we have

$$
g_{i j} \circ g_{j k}=\gamma_{i j k} g_{i k} \quad \text { on }\left.F_{k}\right|_{U_{i j k}}
$$

Therefore we find that $F_{i} / G$ 's are glued to an orbifold. Hence $|\mathcal{F} / G|$ has a structure of an orbifold.

Let $\mathcal{F}$ and $\mathcal{F}^{\prime}$ be vector bundles over $\left(\left\{h_{i j}\right\},\left\{\gamma_{i j k}\right\}\right)$ and $\left(\left\{h_{i j}^{\prime}\right\},\left\{\gamma_{i j k}^{\prime}\right\}\right)$, respectively. Suppose that $\left(\left\{\mu_{i j}\right\},\left\{\psi_{i}\right\}\right):\left(\left\{h_{i j}\right\},\left\{\gamma_{i j k}\right\}\right) \rightarrow\left(\left\{h_{i j}^{\prime}\right\},\left\{\gamma_{i j k}^{\prime}\right\}\right)$ is an isomorphism and $\left\{\phi_{i}\right\}: \mathcal{F} \rightarrow \mathcal{F}^{\prime}$ is an isomorphism over $\left(\left\{\mu_{i j}\right\},\left\{\psi_{i}\right\}\right)$. Then $\phi_{i}$ is a diffeomorphism between global quotient orbifolds $F_{i} / G$ and $F_{i}^{\prime} / G$. Clearly they are glued to a diffeomorphism between orbifolds $\mathcal{F} / G$ and $\mathcal{F}^{\prime} / G$.

If necessary, we take a refinement of $\left\{U_{i}\right\}$ such that $F_{i}$ is $G$-equivariantly isomorphic to $U_{i} \times F$. Note that $G$ acts trivially on $U_{i}$ and effectively on $F$. The open subset $\pi^{-1}\left(U_{i}\right) \subset|\mathcal{F} / G|$ is nothing but $F_{i} / G \cong U_{i} \times F / G$. Therefore $\pi: \mathcal{F} / G \rightarrow M$ is a locally trivial fiber bundle whose fiber is $F / G$.

Example 6.8. Let $\mathcal{F}=\left(\left\{F_{i}\right\},\left\{g_{i j}\right\}\right)$ be a vector bundle over $\left(\left\{h_{i j}\right\},\left\{\gamma_{i j k}\right\}\right) \in$ $\operatorname{Sh}(M, \underline{G})$. By Lemma $6.4 \pi: \mathcal{F} / G \rightarrow M$ is a locally trivial fiber bundle of $C^{\infty}$ class with fiber $\mathbb{R}^{k} / G$, where $g_{i j}: \mathbb{R}^{k} \rightarrow \mathbb{R}^{k}$.

We remark that here we regard $M$ as a manifold and not a stack.

We assume that $g_{i j} \in O(k)$. Namely we assume that it preserves a metric of the fiber. We consider the set of the equivalence classes of all $x \in F_{i}$ with $\|x\| \leq r$ and denote it by $\mathcal{F} / G(r)$. Then the restriction $\pi: \mathcal{F} / G(r) \rightarrow M$ is also a locally trivial fiber bundle of $C^{\infty}$ class, that is a 'ball bundle'. Similarly when we denote the set of all the equivalence classes of $x \in F_{i}$ with $\|x\|=r$ by $S(\mathcal{F} / G)$, it is a locally trivial fiber bundle of $C^{\infty}$ class over $M$ with fiber $S^{n-1} / G$. This is a 'sphere bundle'.

In particular, if $X=\tilde{X} / G$ is a global quotient, then the map $N_{X \cong(\Gamma)} X / \Gamma \rightarrow$ $X^{\cong}(\Gamma)$ defines a locally trivial fiber bundle of $C^{\infty}$ class. We can define 'ball bundle' and 'sphere bundle', that are locally trivial fiber bundles of $C^{\infty}$ class, also in this case. 
Example 6.8 says the normal bundle of $X^{\cong}(\Gamma)$ is locally trivial in $C^{\infty}$ sense. We note that in the theory of stratification of analytic set or of Whitney stratification one important point (observed by Whitney) is that the normal cone to the stratum is locally trivial only in $C^{0}$ sense.

Example 6.9. Let $C_{a}=\left\{(t x, t y, t) \in \mathbb{R}^{3}|-1 \leq x \leq 1,0 \leq y \leq 1+a-a| x \mid, t \geq 0\right\}$ and we put

$$
X=\left\{(x, y, z, w) \in \mathbb{R}^{4} \mid(x, y, z) \in C_{w}, 0<w<1\right\} .
$$

Using the fact that $C_{a}$ is not affine isomorphic to $C_{b}$ for $a \neq b$, we can prove that a neighborhood of $w$ axis in $X$ is not diffeomorphic to the product $\mathbb{R} \times Z$ for any $Z \subset \mathbb{R}^{3}$. (Here we say $X$ is diffeomorphic to $\mathbb{R} \times Z$ if there exists a homeomorphism which extends to a diffeomorphism to its sufficiently small open neighborhoods.)

For the stratification $\left\{X^{\cong}(\Gamma)\right\}$ of the orbifold $X=\widetilde{X} / G$, we have the following:

Lemma 6.5. (Tubular neighborhood theorem) Consider the situation of Example-Definition 5.1. Denote by $B\left(N_{X \cong(\Gamma)} X\right)$ the unit ball bundle of $N_{X \cong(\Gamma)} X$. Then $\left(B\left(N_{X \cong(\Gamma)} X\right)\right) / \Gamma$ is diffeomorphic to a neighborhood of $X^{\cong}(\Gamma)$ in $X$ as an orbifold.

Proof. We use the notation of Definition 6.2. We recall that

$$
N_{X} \cong(\Gamma)=\left(\left\{E_{i}\right\},\left\{g_{i j}\right\}\right)
$$

where $E_{i}=N_{\widetilde{U}_{i}} \widetilde{X}$. Note that $E_{i}$ is the restriction of $N_{\widetilde{X}=(\Gamma)} \widetilde{X}$ to $\widetilde{U}_{i} \subset \widetilde{X}$.

We pick open neighborhoods $W_{1} \supset W_{2} \supset \cdots$ of $\bigcup_{\Gamma^{\prime} \supset \Gamma} X^{\cong}\left(\Gamma^{\prime}\right)$ in $X$ such that $\bigcap_{k} W_{k} \cap X^{\cong}(\Gamma)=\emptyset$. We may assume that $X^{\cong}(\Gamma)_{k}=X^{\cong}(\Gamma) \backslash W_{k}$ is a closed submanifold in $X \backslash W_{k}$. Denote by $\widetilde{X}^{=}(\Gamma)_{k}=\widetilde{X}^{=}(\Gamma) \backslash \pi^{-1}\left(W_{k}\right)$, where $\pi: \widetilde{X} \rightarrow X$ is the projection.

We take a $G$ invariant Riemannian metric on $\widetilde{X}$ and use the exponential map to identify $E_{i}=N_{\widetilde{U}_{i}} \widetilde{X}$ with a neighborhood of $\widetilde{U}_{i}$ in $\widetilde{X}$ as follows.

Denote by $B_{\delta}\left(N_{\widetilde{X}=(\Gamma)} \tilde{X}\right)$ the ball bundle of $N_{\widetilde{X}=(\Gamma)} \tilde{X}$ of radius $\delta$. For $k=$ $1,2, \ldots$, we take $\delta_{k}>0$ such that the exponential map

$$
\exp : v \in B_{\delta_{k}}\left(\left.N_{\widetilde{X}=(\Gamma)} \widetilde{X}\right|_{\widetilde{X}=(\Gamma)_{k}}\right) \mapsto \exp (v) \in \widetilde{X}
$$

which is a diffeomorphism to an open neighborhood of $\widetilde{X}^{=}(\Gamma)_{k}$. 
We pick an $H(\Gamma)$-invariant smooth positive function $\chi: \widetilde{X}^{=}(\Gamma) \rightarrow \mathbb{R}$ such that $\chi<\delta_{k+1}$ on $\widetilde{X}^{=}(\Gamma) \cap \pi^{-1}\left(W_{k}\right)$. Denote by $p_{i}: E_{i} \rightarrow \widetilde{U}_{i}$ the projection and set $B_{\chi}\left(E_{i}\right)=\left\{v \in E_{i} \mid\|v\|<\chi\left(p_{i}(v)\right)\right\}$. Then the exponential map exp restricted to $B_{\chi}\left(E_{i}\right)$ induces a diffeomorphism from $B_{\chi}\left(E_{i}\right) / G$ to an open neighborhood of $U_{i}$ in $X$ as orbifolds. Clearly, we find that $\left.\underline{h}_{i j} \circ \exp \right|_{B_{\chi}\left(E_{j}\right)}=\left.\exp \right|_{B_{\chi}\left(E_{i}\right)} \circ g_{i j}$ on $E_{j} \mid \underline{h}_{i j}^{-1}\left(\widetilde{U}_{i}\right) \cap \widetilde{U}_{j}$. Hence they are glued together and we obtain a diffeomorphism $\exp (\chi \cdot \bullet)$ from $\left(B\left(N_{X \cong(\Gamma)} X\right)\right) / \Gamma$ to an open neighborhood of $X^{\cong}(\Gamma)$.

Finally we remark that various operations on vector bundles such as Whitney sum, tensor product, Hom bundle, symmetric tensor product, etc. can be generalized to the case of vector bundles on $\left[\left\{h_{i j}\right\},\left\{\gamma_{i j k}\right\}\right] \in S h(M ; \underline{G})$. For example, if $\mathcal{F}=\left(\left\{F_{i}\right\},\left\{g_{i j}^{F}\right\}\right)$ and $\mathcal{E}=\left(\left\{E_{i}\right\},\left\{g_{i j}^{E}\right\}\right)$ are vector bundles on $\left(\left\{h_{i j}\right\},\left\{\gamma_{i j k}\right\}\right)$ then $\operatorname{Hom}(\mathcal{F}, \mathcal{E})=\left(\left\{\operatorname{Hom}\left(F_{i}, E_{i}\right)\right\},\left\{g_{i j}\right\}\right)$ where

$$
g_{i j}\left(u_{j}\right)=g_{i j}^{E} \circ u_{j} \circ\left(g_{i j}^{F}\right)^{-1} .
$$

We use the next lemma in Section 9.

Lemma 6.6. Let $\mathcal{E}=\left(\left\{E_{i}\right\},\left\{g_{i j}\right\}\right)$ be a vector bundle on $\left(\left\{h_{i j}\right\},\left\{\gamma_{i j k}\right\}\right) \in$ $\operatorname{Sh}(M, \underline{G})$. We put

$$
E_{i}^{G}=\left\{v \in E_{i} \mid \forall \gamma \in G \gamma v=v\right\}
$$

Then they are glued by $g_{i j}$ to define a vector bundle on the topological space $M$ (in the usual sense).

Proof. Let $g_{i j}^{G}:\left.E_{i}^{G}\right|_{U_{i j}} \rightarrow E_{j}^{G}$ be the restriction of $g_{i j}$. Since $G$ action on $E_{i}^{G}$ is trivial, it follows from (23) that

$$
g_{i j}^{G} \circ g_{j k}^{G}=g_{i k}^{G}
$$

The lemma follows.

Remark 6.10. In some situation we need to consider subspaces $Y_{p} \subset V_{p}$ of a space $X$ with Kuranishi structure $\left(V_{p}, E_{p}, \Gamma_{p}, \psi_{p}, s_{p}\right)$ and put a Kuranishi structure on the subspace. When the action of $\Gamma_{p}$ is not effective, we need to add some $\Gamma_{p}$ vector space $F_{p}$ to both $Y_{p}$ and obstruction bundle $E_{p}$ of each Kuranishi neighborhood, in order to define a Kuranishi structure on the subspace $\cup_{p} Y_{p}$. We 
need some care to carry this out. Namely we should glue those vector spaces by a family of linear isomorphisms $g_{p q}: U_{p q} \rightarrow \operatorname{Hom}\left(F_{q}, F_{p}\right)$ so that

$$
g_{p q} \circ g_{q r}=\gamma_{p q r} \cdot g_{p r}
$$

where $\gamma_{p q r}$ is the one appearing in Definition 2.3 (ii). For example, in the case of Kuranishi structure with corners, we define Kuranishi structure on codimension $k$ corner $S_{k} X$ by taking the normal bundle of $S_{k} X$ and adding the normal bundle to both $S_{k} V_{p}$ and the obstruction bundle in order to make the $\Gamma_{p}$ action effective.

\section{Single Valued Piecewise Smooth Section of orbi-Bundle: \\ STATEMENT}

Let $X=\tilde{X} / G$ be a global quotient. We consider the situation of ExampleDefinition 5.1. We decompose $X^{\cong}(\Gamma)$ into the connected components

$$
X^{\cong}(\Gamma)=\bigcup_{i} X^{\cong}(\Gamma ; i) .
$$

Let $[p] \in X^{\cong}(\Gamma ; i)$ where $p \in \widetilde{X}$. Let $E \rightarrow \widetilde{X}$ be a $G$ equivariant vector bundle. It is, by definition, an orbi-bundle $E / G$ on $X$. A single valued section of this orbi-bundle is, by definition, a $G$-equivariant section of $E \rightarrow \widetilde{X}$.

We have a $\Gamma$ action on the fiber $E_{p}$ of our vector bundle $E$. In this situation we recall from Definition 3.2 :

$$
\begin{aligned}
E_{p}^{\Gamma} & =\left\{v \in E_{p} \mid \forall \gamma \in \Gamma \gamma v=v\right\} . \\
d(X ; \Gamma ; i) & =\operatorname{dim} X^{\cong}(\Gamma ; i)-\operatorname{dim} E_{p}^{\Gamma} .
\end{aligned}
$$

In Sections 8-9 we will prove the following:

Proposition 7.1. For each $C^{0}$-section $s$ of the orbi-bundle $E / G \rightarrow X$, there exists a sequence of single valued piecewise smooth sections $s_{\epsilon}$ converging to $s$ in $C^{0}$-sense such that the following holds:

(i) $s_{\epsilon}^{-1}(0)$ has a smooth triangulation. Namely for each simplex the embedding $\Delta \rightarrow X$ locally lifts to a map to $\widetilde{X}$ which is a smooth embedding.

(ii) $s_{\epsilon}^{-1}(0) \cap X^{\cong}(\Gamma)$ is a $P L$ manifold such that each simplex is smoothly embedded into $X^{\cong}(\Gamma)$.

(iii) If $\Delta$ is a simplex of $(i)$ whose interior intersects with $X^{\cong}(\Gamma)$, the intersection of $\Delta$ with $X^{\cong}(\Gamma)$ is $\Delta$ minus some faces and is smoothly embedded in $X^{\cong}(\Gamma)$. 
(iv)

$$
\operatorname{dim} s_{\epsilon}^{-1}(0) \cap X^{\cong}(\Gamma ; i)=\operatorname{dim} X^{\cong}(\Gamma ; i)-\operatorname{dim} E_{p}^{\Gamma} .
$$

Remark 7.1. We note that if $s$ is a single-valued section and if $[p] \in X^{\cong}(\Gamma)$ then $s(p) \in E_{p}^{\Gamma}$. So the dimension given in (iv) is optimal.

We will use Proposition 7.1 in the proof of Theorem 3.1 in Section 10.

\section{System of tubular neighborhoods}

The proof of Proposition 7.1 is closely related to the proof of existence of a triangulation of the space with Whitney stratification. (See [16]). Especially, we use the notion of a system of tubular neighborhoods introduced by Mather [20]. Mather used this notion to prove the famous first isotopy lemma (see 8 in Section II [20]). The first isotopy lemma implies that Whitney stratification has a $C^{0}$ locally trivial normal cone. (See Theorem 8.3 [20]). Note that existence of smooth triangulation of an orbifold is well-known which is not what we intend to prove. In order to show that the zero set of our section has a smooth triangulation, we use various constructions appearing in the proof of existence of a $C^{0}$-triangulation on the Whitney stratified space.

As we explained in Example 6.8, we have a $C^{\infty}$ locally trivial tubular neighborhood in our situation. This property makes the system of tubular neighborhoods (or the system of normal cones) in this case carry some properties better than that of [20].

We follow Section II in [20] in the next definition.

Definition 8.1. A tubular neighborhood of the stratum $X^{\cong}(\Gamma)$ in $X$ is a quadruple $\left(\pi_{\Gamma}, N_{X \cong(\Gamma)} X, \sigma, \phi\right)$ satisfying

(i) $\pi_{\Gamma}: N_{X \cong(\Gamma)} X \rightarrow X^{\cong}(\Gamma)$ is a vector bundle in the sense of stack. (See Definition 6.1.)

(ii) $\sigma: X^{\cong}(\Gamma) \rightarrow \mathbb{R}_{+}$is a smooth positive function.

(iii) $\phi: B_{\sigma}(\Gamma) / \Gamma \rightarrow U^{\cong}(\Gamma)$ is a diffeomorphism (in the sense of Definition 6.5) onto a neighborhood $U \cong(\Gamma)$ of $X^{\cong}(\Gamma)$ in $X$. Here $B_{\sigma}(\Gamma)=\left\{v \in N_{X \cong(\Gamma)} X \mid\right.$ $\left.\|v\|<\sigma\left(\pi_{\Gamma}(v)\right)\right\}$. 
We define $\pi_{\Gamma}: U^{\cong}(\Gamma) \rightarrow X^{\cong}(\Gamma)$ as the composition $\pi_{\Gamma} \circ \phi^{-1}$. We also define $\rho_{\Gamma}^{\prime}: U^{\cong}(\Gamma) \rightarrow \mathbb{R}$ by

$$
\rho_{\Gamma}^{\prime}(\phi(v))=\|v\|^{2} .
$$

We call $\rho_{\Gamma}^{\prime}$ the tubular distance function. We note that both maps are smooth and $\pi_{\Gamma}$ is a submersion. Moreover the pair

$$
\left(\pi_{\Gamma}, \rho_{\Gamma}^{\prime}\right): U^{\cong}(\Gamma) \backslash X^{\cong}(\Gamma) \rightarrow X^{\cong}(\Gamma) \times \mathbb{R}_{>0}
$$

defines a locally trivial fiber bundle of $C^{\infty}$ class.

We need to adjust them so that they become compatible for different $\Gamma$ 's.

Remark 8.2. In the situation of [20], the maps $\pi_{\Gamma}, \rho_{\Gamma}^{\prime}$ are smooth only in the interior of the stratum.

Definition 8.3. A system of tubular neighborhoods of our stratification $\left\{X^{\cong}(\Gamma) \mid\right.$ $\Gamma\}$ is a family $\left(\pi_{\Gamma}, \rho_{\Gamma}^{\prime}\right)$ such that

$$
\begin{aligned}
& \pi_{\Gamma^{\prime}} \circ \pi_{\Gamma}=\pi_{\Gamma^{\prime}} \\
& \rho_{\Gamma^{\prime}}^{\prime} \circ \pi_{\Gamma}=\rho_{\Gamma^{\prime}}^{\prime}
\end{aligned}
$$

holds for $\Gamma^{\prime} \supset \Gamma$. Here we assume the equalities $(30 a)$, (30b) whenever both sides are defined.

Proposition 8.1. There exists a system of tubular neighborhoods.

The proof is actually the same as that of Corollary 6.5 of [20]. Mather proved the existence of a system of tubular neighborhoods for the space with Whitney stratification. In his case the situation is less tame than our case since the normal cone exists only in $C^{0}$ sense. (See example 6.9.) In our case, the proof is easier since the normal cone we produce by Lemma 6.5 is already smooth. For the sake of completeness, we give the proof of Proposition 8.1 later in this subsection (Proposition 8.3).

We next define the notion of a family of lines following Goresky [16]. For $\epsilon>0$ we put:

$$
\begin{aligned}
S^{\Gamma}(\epsilon) & =\left\{p \in U^{\cong}(\Gamma) \mid \rho_{\Gamma}(p)=\epsilon^{2}\right\} \\
U^{\cong}(\Gamma)(\epsilon) & =\left\{p \in U^{\cong}(\Gamma) \mid \rho_{\Gamma}(p)<\epsilon^{2}\right\} .
\end{aligned}
$$

Here we modify $\rho_{\Gamma}^{\prime}$ to $\rho_{\Gamma}$ in the following way. (See the lines 2-5 from the bottom of [16] p 193. We warn that our notation $\rho_{\Gamma}$ corresponds to Goresky's $\rho_{X}^{\prime}$ and $\rho_{\Gamma}^{\prime}$ 
to Goresky's $\rho_{X}$.) We take $\rho_{\Gamma}^{\prime}(p)=\|v\|^{2}$ for $p=\phi(v)$ where $\|\cdot\|$ is an appropriate norm induced by an inner product. We take a function $f_{\Gamma}: X^{\cong}(\Gamma) \rightarrow \mathbb{R}_{+}$that goes to infinity on the boundary. Then we define $\rho_{\Gamma}(x)=\left(f_{\Gamma} \circ \pi_{\Gamma}(x)\right) \rho_{\Gamma}^{\prime}(x)$. (Note $f$ is related to $\sigma$ in Definition 8.1 by $\sigma=\epsilon^{2} / f^{2}$.)

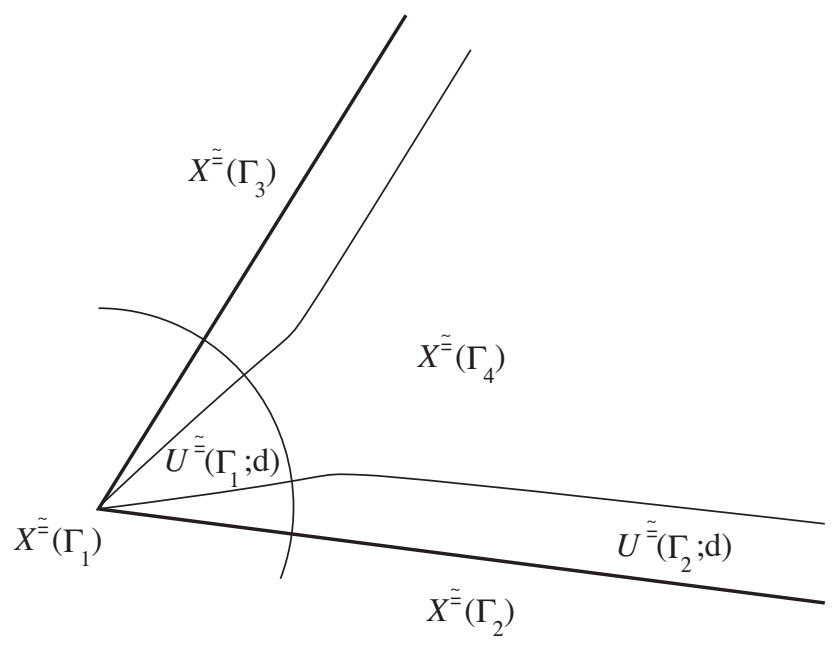

Figure 1

Definition 8.4. A family of smooth maps

$$
r_{\Gamma}(\epsilon): U^{\cong}(\Gamma) \backslash X^{\cong}(\Gamma) \rightarrow S^{\Gamma}(\epsilon)
$$

is said to be a family of lines if the following holds for $\Gamma^{\prime} \supset \Gamma$ :

(i) $r_{\Gamma^{\prime}}\left(\epsilon^{\prime}\right) \circ r_{\Gamma}(\epsilon)=r_{\Gamma}(\epsilon) \circ r_{\Gamma^{\prime}}\left(\epsilon^{\prime}\right) \in S^{\Gamma}(\epsilon) \cap S^{\Gamma^{\prime}}\left(\epsilon^{\prime}\right)$ for all $\epsilon^{\prime}, \epsilon>0$.

(ii) $\rho_{\Gamma^{\prime}} \circ r_{\Gamma}(\epsilon)=\rho_{\Gamma^{\prime}}$.

(iii) $\rho_{\Gamma} \circ r_{\Gamma^{\prime}}(\epsilon)=\rho_{\Gamma}$.

(iv) $\pi_{\Gamma^{\prime}} \circ r_{\Gamma}(\epsilon)=\pi_{\Gamma^{\prime}}$.

(v) If $0<\epsilon<\epsilon^{\prime}<\delta$ then $r_{\Gamma}\left(\epsilon^{\prime}\right) \circ r_{\Gamma}(\epsilon)=r_{\Gamma}\left(\epsilon^{\prime}\right)$.

(vi) $\pi_{\Gamma} \circ r_{\Gamma}(\epsilon)=\pi_{\Gamma}$.

(vii) We define

$$
h_{\Gamma}: U^{\cong}(\Gamma)(\epsilon) \backslash X^{\cong}(\Gamma) \rightarrow S^{\Gamma}(\epsilon) \times(0, \epsilon)
$$

by

$$
h_{\Gamma}(p)=\left(r_{\Gamma}(\epsilon)(p), \sqrt{\rho_{\Gamma}(p)}\right)
$$


and extend it to a map from $U^{\cong}(\Gamma ; \epsilon)$ to the mapping cone of

$$
\left.\pi_{\Gamma}\right|_{S^{\Gamma}(\epsilon)}: S^{\Gamma}(\epsilon) \rightarrow X^{\cong}(\Gamma),
$$

by setting $h_{\Gamma}(p)=(p, 0)$ on $X^{\cong}(\Gamma)$. Then $h_{\Gamma}$ becomes a diffeomorphism.

Note the mapping cone of $\left.\pi_{\Gamma}\right|_{S^{\Gamma}(\epsilon)}: S^{\Gamma}(\epsilon) \rightarrow X^{\cong}(\Gamma)$ is identified with $\{p \epsilon$ $\left.U^{\cong}(\Gamma) \mid \rho_{\Gamma}(p) \leq \epsilon^{2}\right\}$. So it is an orbifold with boundary. (It is a total space of the ball bundle discussed in Example 6.8.) We remark that the diffeomorphism in (vii) is one in the sense of Definition 6.5.

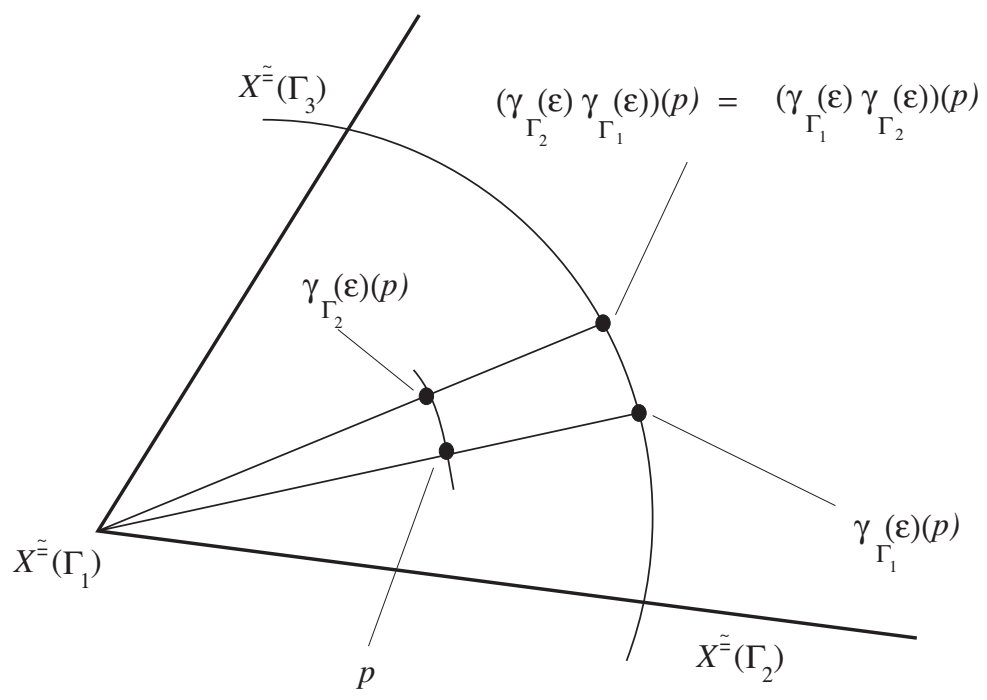

Figure 2

The above definition except (vii) exactly coincides with that of Goresky [16]. The condition (vii) is stronger than the corresponding one from [16]. This is because in our situation the normal cone is smooth and diffeomorphic to a neighborhood $U^{\cong}(\Gamma)$ of $X^{\cong}(\Gamma)$.

Proposition 8.2. There exists a family of lines.

We can prove Proposition 8.2 in the same way as in [16] except that we need some extra argument to check (vii). Instead of working this out, we give a slightly different self-contained proof of Propositions 8.1 and 8.2 below, which exploits the special case of orbifolds (or the space with a stratification whose strata have locally trivial normal bundles of $C^{\infty}$-class). The proof below is simpler than 
those by Mather or Goresky. This is because we have already proved that there exists a normal cone which is $C^{\infty}$ locally trivial. For the cases studied by Mather or Goresky, proving existence of $C^{0}$ trivial normal cone is one of the main goals of their study. So our proof here rather goes in the direction opposite to their study.

Now we will prove the relative versions of Propositions 8.1 and 8.2 below which include the propositions themselves. Hereafter we write $(\pi, \rho, r)$ in place of $\left\{\left(\pi_{\Gamma}, \rho_{\Gamma}, r_{\Gamma}\right) \mid \Gamma \subset G\right\}$ for simplicity. We also write $\pi \circ r=\pi$ etc. in place of Definition 8.4 (vi) etc. by an abuse of notations.

Proposition 8.3. Let $X$ be a global quotient and $K$ a compact subset of $X$. Assume that there exist a system of tubular neighborhoods $(\pi, \rho)$ and a family of lines $r$ in a neighborhood $U$ of $K$. Then there exist $\pi, \rho$ and $r$ on $X$ that coincide with the given ones in a neighborhood of $K$ respectively.

For the proof of Proposition 8.3, we generalize it to the following relative version. (Compare this with 6 in Section II [20] where a similar procedure of the proof is applied.)

Proposition 8.4. Let $X$ be a global quotient and $K$ a compact subset of $X$. Let $U_{1}, U_{4}$ be open subsets of $X$ such that $U_{4} \supset K$ and $U_{1} \supset \bar{U}_{4}$. Assume that there exist a system of tubular neighborhoods $(\pi, \rho)$ and a family of lines $r$ on $U_{1}$. We also assume that there exists a locally trivial fiber bundle of $C^{\infty}$ class $p r_{N}: X \backslash U_{4} \rightarrow N$ where $N$ is a manifold. We assume $p r_{N} \circ \pi=p r_{N}$, and $p r_{N} \circ r=p r_{N}$ on $U_{1} \backslash U_{4}$.

Then there exist open sets $U_{2}, U_{3}$ with $U_{j} \supset \bar{U}_{j+1}$ for $j=1,2,3$ and there exist $\pi, \rho$ and $r$ on $X$ which coincide with the given ones on a neighborhood of $K$ in $\bar{U}_{4}$. In addition, $p r_{N} \circ r=p r_{N}$ and $p r_{N} \circ \pi=p r_{N}$ hold on $U_{2} \backslash U_{3}$.

Again we write $p r_{N} \circ \pi=p r_{N}$ etc. in place of $p r_{N} \circ \pi_{\Gamma}=p r_{N}$ etc. by an abuse of notations.

Proof. By shrinking $U_{1}$ if necessary, we may assume that $X^{\cong}(\Gamma ; i) \subset U_{4}$ if and only if $X^{\cong}(\Gamma ; i) \subset U_{1}$ for each $\Gamma$. We put

$$
\begin{aligned}
D & =\operatorname{dim} X-\inf \left\{\operatorname{dim} X^{\cong}(\Gamma ; i) \mid X^{\cong}(\Gamma ; i) \text { is not contained in } U_{1}\right\} \\
& =\sup \left\{\operatorname{codim} X^{\cong}(\Gamma ; i) \mid X^{\cong}(\Gamma ; i) \text { is not contained in } U_{1}\right\} .
\end{aligned}
$$


The proof is given by an induction over $D$. If $D$ is 0 , there is nothing to prove.

We assume that Proposition 8.4 is proved when (33) is $D-1$ or smaller and prove the case of $D$. Let $X^{\cong}(\Gamma ; i)$ be a stratum of codimension $D$. Since this is the stratum of smallest dimension in $X \backslash U_{4}$, it follows that it is a smooth manifold outside $U_{4}$. We put $X_{0}^{\cong}(\Gamma ; i)=X^{\cong}(\Gamma ; i) \backslash U_{3.5}$. Here $U_{3.5}$ is a neighborhood of $K$, which is slightly bigger than $U_{4}$.

By Example 6.8, we have the locally trivial fiber bundle of $C^{\infty}$ class

$$
\partial N_{X_{0}^{\cong}(\Gamma ; i)} X / \Gamma \longrightarrow X_{0}^{\cong}(\Gamma ; i) .
$$

By Lemma 6.5 (see also Mather's version of tubular neighborhood theorem in p.213 [20]) we may take the projection $\pi_{\Gamma}: N_{X_{0}^{\cong}(\Gamma ; i)} X / \Gamma \rightarrow X_{0}^{\cong}(\Gamma ; i)$ so that $p r_{N} \circ \pi_{\Gamma}=p r_{N}$, by choosing the Riemannian metric we use to prove Lemma 6.5 so that each of the fibers of $p r_{N}$ is totally geodesic.

We will now modify the tubular neighborhood and the family of lines on $\left(U_{1} \backslash\right.$

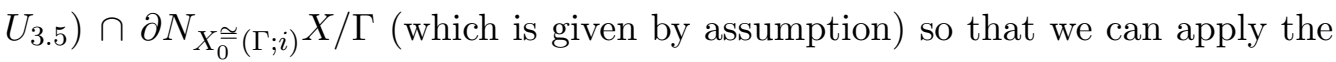

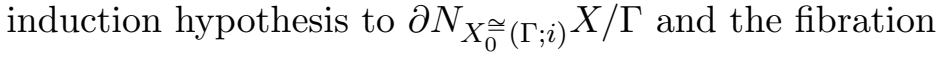

$$
\pi_{\Gamma}: \partial N_{X_{0}}(\Gamma ; i)=X_{0}^{\cong}(\Gamma ; i) .
$$

Note when we apply the induction hypothesis $X_{0}^{\cong}(\Gamma ; i)$ plays the role of $N$ and

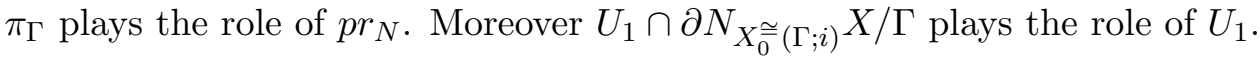

To apply the induction hypothesis to (35), we need to check that the assumption is satisfied. In particular we need to find a tubular neighborhood and a family of lines on $U_{1} \cap \partial N_{X} \cong(\Gamma ; i) X / \Gamma$ that is compatible with $\pi_{\Gamma}$.

By the assumption of Proposition 8.4, there exist a tubular neighborhood and a family of lines $\left(\pi^{\prime}, \rho^{\prime}, r^{\prime}\right)$ on the set $U_{1}$ that is compatible with $p r_{N}$. It induces a tubular neighborhood and a family of lines $\left(\pi^{\prime}, \rho^{\prime}, r^{\prime}\right)$ on the set $\partial N_{X_{0}^{\cong}(\Gamma ; i)} X / \Gamma \cap$ $U_{1}$. This is compatible with $p r_{N}$ but not necessarily compatible with $\pi_{\Gamma}$. We claim that we can modify $\pi_{\Gamma}$ so that it is compatible with $\left(\pi^{\prime}, \rho^{\prime}, r^{\prime}\right)$ on the set $\partial N_{X}^{\cong}(\Gamma ; i)=/ \Gamma \cap U_{1}$.

Since there exists $\left(\pi^{\prime}, \rho^{\prime}, r^{\prime}\right)$ on the set $U_{1}$, there exists

$$
\pi_{\Gamma}^{\prime}: \partial N_{X} \cong(\Gamma ; i)=/ \Gamma \rightarrow X_{0}^{\cong}(\Gamma ; i)
$$

such that if $\Gamma \supset \Gamma^{\prime}$, the map $\pi_{\Gamma}^{\prime}$ is consistent with $\pi_{\Gamma^{\prime}}^{\prime}, \rho_{\Gamma^{\prime}}^{\prime}$ and $r_{\Gamma^{\prime}}^{\prime}$ in the sense of (30) and Definition 8.4 (iv). 
We note that $\pi_{\Gamma}^{\prime}$ may not coincide with $\pi_{\Gamma}$ given by Lemma 6.5. But we can modify and glue them as follows: The difference between two projections $\left(\pi_{\Gamma}^{\prime}\right.$ above and $\pi_{\Gamma}$ ) can be chosen to be arbitrarily small (in $C^{1}$ sense), by taking the tubular neighborhood small. Then we can use the minimal geodesic of a Riemannian metric on $X_{0}^{\cong}(\Gamma ; i)$, to find an isotopy between them. Hence by a standard argument we can glue them. We thus proved the claim.

Thus we can apply our induction hypothesis to (35) and obtain the system $(\pi, r, \rho)$ on $\partial N_{X_{0}^{\cong}(\Gamma ; i)} X / \Gamma$. Since $N_{X_{0}^{\cong}(\Gamma ; i)} X / \Gamma \cong U\left(X_{0}^{\cong}(\Gamma)\right)$ is a cone of $\partial N_{X_{0} \cong(\Gamma ; i)} X / \Gamma$, the system $(\pi, r, \rho)$ on $\partial N_{X_{0}^{\cong}(\Gamma ; i)} X / \Gamma$ induces one on $N_{X_{0}^{\cong}(\Gamma ; i)} X / \Gamma$ in an obvious way. It commutes with the projection $U\left(X_{0}^{\cong}(\Gamma)\right) \rightarrow N$, since $U\left(X_{0}^{\cong}(\Gamma)\right) \rightarrow N$ factors through $\pi_{\Gamma}$.

Recall that we have $(\pi, r, \rho)$ on $U_{1}$ by assumption. On $U_{1} \cap U\left(X_{0}^{\cong}(\Gamma)\right)$, this system may not coincide with the one we have just constructed above. We now explain how we adjust this system to carry out the gluing process.

We first note that the projection $\pi_{\Gamma}: \partial N_{X_{0}^{\cong}(\Gamma ; i)} X / \Gamma \rightarrow X_{0}^{\cong}(\Gamma ; i)$ coincides for the two systems on $U_{1}$ since they are already arranged so when we apply the induction hypothesis above. Since both systems on $U_{1}$ are the cone of the same system $(\pi, \rho, r)$ on $\partial N_{X_{0}^{\cong}(\Gamma ; i)} X / \Gamma$ by the same map, it follows that they are the same as an abstract structure.

However, the diffeomorphism from the cone of $\partial N_{X_{0}}(\Gamma ; i) X / \Gamma$ to $U\left(X_{0}^{\cong}(\Gamma ; i)\right)$ (which exists by (vii)) may not coincide. (Note this map is defined by $r$, the family of lines of each of the structures.) But we can show that they are isotopic by the same method as before. Namely, we go to a branched covering of the cone

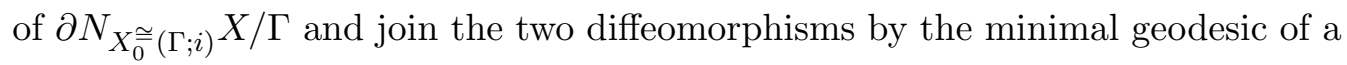
$G$-equivariant Riemannian metric that is totally geodesic along the fiber of $p r_{N}$. Therefore we can glue them by the standard method.

Now we have extended the systems $(\pi, \rho, r)$ to a neighborhood of $X^{\cong}(\Gamma ; i)$. We repeat the same construction for each $X^{\cong}(\Gamma ; i)$ with $\operatorname{dim} X^{\cong}(\Gamma ; i)=\operatorname{dim} X-D$.

We thus reduce the problem to the case when $D$ is strictly smaller. The proof of Proposition 8.4 is now finished by induction. 
9. Single valued Piecewise Smooth Section of orbi-Bundle: Proof

Proof of Proposition 7.1. Let us fix a sufficiently small $d>0$ and put

$$
\operatorname{Int} X^{d}(\Gamma)=X^{\cong}(\Gamma) \backslash \bigcup_{\Gamma^{\prime} \supset \Gamma} \operatorname{Int} U^{\cong}\left(\Gamma^{\prime}\right)(d) .
$$

By definition of system of tubular neighborhoods we observe the following:

$$
U^{\cong}\left(\Gamma_{1}\right) \cap U^{\cong}\left(\Gamma_{2}\right) \neq \emptyset \Rightarrow \Gamma_{1} \subset \Gamma_{2} \text { or } \Gamma_{2} \subset \Gamma_{1} .
$$

It follows from (37) that $\operatorname{Int} X^{d}(\Gamma)$ is a smooth manifold with corners. The codimension $k$ corner of $\operatorname{Int} X^{d}(\Gamma)$ is a union of

$$
X\left(\Gamma ; \Gamma_{1}, \Gamma_{2}, \ldots, \Gamma_{k}\right)=X^{\cong}(\Gamma) \cap \bigcap_{i=1}^{k} S^{\Gamma_{i}}(d)
$$

where

$$
\Gamma_{1} \supset \Gamma_{2} \supset \cdots \supset \Gamma_{k} \supset \Gamma .
$$

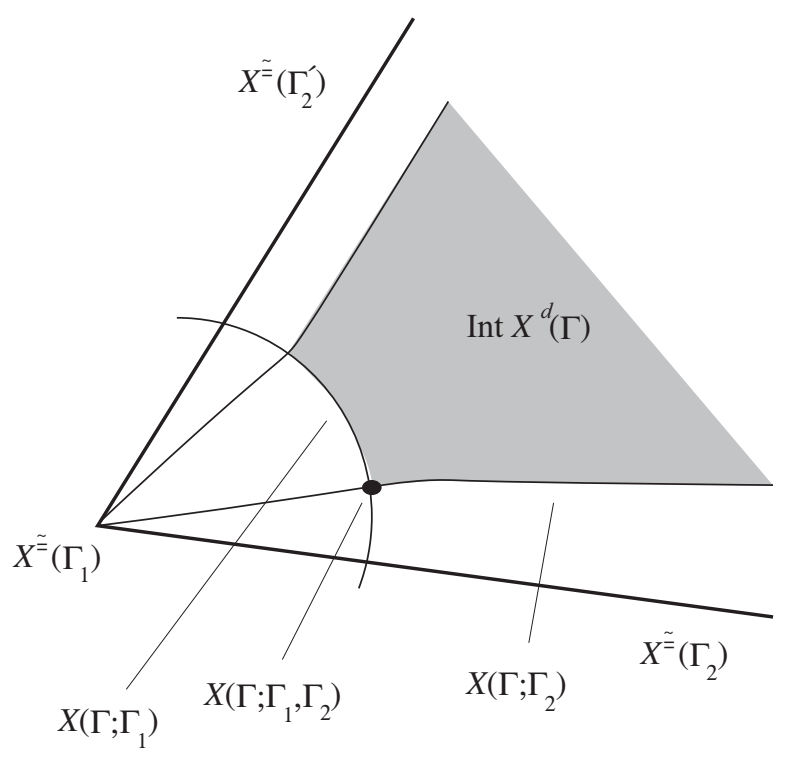

Figure 3

Later in this section, we will first define our section $s_{\epsilon}$ on $\bigcup_{\Gamma} \operatorname{Int} X^{d}(\Gamma)$ and then extend it so that its zero set is a cone with respect to the family of lines. Thus our proof is an analog to the proof given in $\S 3-5[16]$. 
Let $E_{p}^{\Gamma}$ be as in (11). We put:

$$
E_{p}=E_{p}^{\Gamma} \oplus E_{p}^{\perp}
$$

where $E_{p}^{\perp}$ is the complement of $E_{p}^{\Gamma}$ in $E_{p}$.

On $X\left(\Gamma ; \Gamma_{1}, \Gamma_{2}, \ldots, \Gamma_{k}\right)$ we define $E_{p}(\Gamma)$ inductively on $\# \Gamma$ and decompose $E_{p}^{\Gamma}$ into

$$
E_{p}^{\Gamma}=\bigoplus_{i=1}^{k} E_{p}\left(\Gamma_{i}\right) \oplus E_{p}(\Gamma)
$$

as follows.

If $\Gamma$ is maximal, we set $E_{p}(\Gamma)=E_{p}^{\Gamma}$ on $X^{\cong}(\Gamma)$. Using local triviality of $E(\Gamma)$, we extend our subbundle $E(\Gamma)$ to the neighborhood $U^{\cong}(\Gamma)(d)$ for a sufficiently small $d$ so that

$$
E_{p}(\Gamma) \subset E_{p}^{\Gamma^{\prime}}
$$

is satisfied for $p \in U^{\cong}(\Gamma)(d) \cap X^{\cong}\left(\Gamma^{\prime}\right)$, and $\Gamma \supset \Gamma^{\prime}$ as follows:

We take a $\Gamma$-invariant connection $\nabla$ of $E$ on $U^{\cong}(\Gamma)(d)$ so that each of $E^{\Gamma}$ is a totally geodesic subbundle and that the curvature of $\nabla$ is zero on each fiber of $U^{\cong}(\Gamma)(d) \rightarrow X^{\cong}(\Gamma)$. Then we can use the parallel transport with respect to $\nabla$ along the path contained in the fiber of $U^{\cong}(\Gamma)(d) \rightarrow X^{\cong}(\Gamma)$ to extend $E(\Gamma)$ to $U^{\cong}(\Gamma)(d)$.

We next consider $p \in X\left(\Gamma ; \Gamma_{1}\right)$. We may assume that $E_{p}\left(\Gamma_{1}\right)$ is defined. Then we define $E_{p}(\Gamma)$ as the orthonormal complement of $E_{p}\left(\Gamma_{1}\right)$ in $E_{p}^{\Gamma}$. We extend them to its neighborhood. We thus obtain

$$
E_{p} \cong E_{p}\left(\Gamma_{1}\right) \oplus E_{p}(\Gamma) \oplus E_{p}^{\perp} .
$$

We can continue by a downward induction on $\# \Gamma, k$ and obtain the decomposition (41).

We use (41) to perform our construction of $s_{\epsilon}$. We also need the following lemma.

Lemma 9.1. Let $f: M \rightarrow N$ be a locally trivial fiber bundle of $C^{\infty}$ class between smooth manifolds and $F$ a vector bundle on $M$. We fix a smooth triangulation of $N$. Let $s$ be a section of $F$. Then there exists a family $s^{\epsilon}$ of piecewise smooth sections of $F$ such that

(i) $s^{\epsilon}$ converges to $s$ in $C^{0}$ topology. 
(ii) $s^{\epsilon}$ is of general position to 0 .

(iii) $f:\left(s^{\epsilon}\right)^{-1}(0) \rightarrow N$ is piecewise linear with respect to some smooth triangulation of $\left(s^{\epsilon}\right)^{-1}(0)$ and a subdivision of the given triangulation of $N$.

Proof. Since $f$ is a locally trivial fiber bundle of $C^{\infty}$ class, we may choose a triangulation of $M$ and a subdivision of the given one on $N$ with respect to which $f$ is piecewise linear. (See [21], [23].) In other words, there exist simplicial complexes $K_{M}, K_{N}$ and homeomorphisms $i_{M}:\left|K_{M}\right| \rightarrow M, i_{N}:\left|K_{N}\right| \rightarrow N$ with the following properties:

(i) The restrictions of the homeomorphisms $i_{M}$ and $i_{N}$ to each simplex are diffeomorphisms onto their images.

(ii) $i_{N}^{-1} \circ f \circ i_{M}$ is induced by a simplicial map. Namely it sends a simplex of $K_{M}$ to a simplex of $K_{N}$ and is affine on each simplex.

We next take a smooth triangulation of the total space $F$ of our vector bundle so that the projection $F \rightarrow M$ is piecewise linear. By taking an appropriate subdivision of the simplicial decomposition, we may approximate our section $s$ by a section $s^{\epsilon}: M \rightarrow F$ which is piecewise linear, $C^{0}$ close to $s$, and of general position to the zero section. (Existence of such $s^{\epsilon}$ is a standard result of piecewise linear topology. See, for example, [19].) Then (i) and (ii) are satisfied. Since $s^{\epsilon}$ is piecewise linear, which is affine on each simplex, it follows that the intersection of $\left(s^{\epsilon}\right)^{-1}(0)$ with each simplex is affine. Hence we can find a subdivision of $K_{M}$ and $K_{N}$ such that $\left(s^{\epsilon}\right)^{-1}(0)$ is a subcomplex and the restriction of $f$ to $\left(s^{\epsilon}\right)^{-1}(0)$ is piecewise linear.

We note that Lemma 9.1 (iii) implies that the mapping cone of $f:\left(s^{\epsilon}\right)^{-1}(0) \rightarrow$ $N$ has a smooth triangulation.

Now we start the construction of our section $s_{\epsilon}$. We will put

$$
s_{\epsilon}=\bigoplus_{i} s_{\epsilon}^{\Gamma_{i}} \oplus s_{\epsilon}^{\Gamma} \oplus 0
$$

according to our decomposition (40), (41). Note the $E_{p}^{\perp}$-component is necessarily zero because of the $G$-invariance. (In other words it is zero since $s_{\epsilon}$ is singlevalued.)

We will construct $s_{\epsilon}^{\Gamma}$ by the downward induction over the order of $\Gamma$. 
Let $\Gamma$ be maximal. We consider the vector bundle $E^{\Gamma} \rightarrow X^{\cong}(\Gamma)$. We note that this is a vector bundle on a manifold and is not only an orbi-bundle. So for each given section $s$ we can take a smooth section $s_{\epsilon}^{\Gamma}$ which is transversal to 0 and $C^{0}$ close to $s$. We extend $s_{\epsilon}^{\Gamma}$ to a section of $E(\Gamma)$ on $U^{\cong}(\Gamma)$ so that it is covariantly constant along the fiber of $\pi_{\Gamma}$.

We next consider the case of $\Gamma$ that is not necessarily maximal but has a property that there is no $\Gamma_{1} \supset \Gamma_{2} \supset \Gamma$ with $X\left(\Gamma ; \Gamma_{1}, \Gamma_{2}\right) \neq \emptyset$. We consider $\operatorname{Int} X^{d}(\Gamma) \cap S^{\Gamma_{1}}(d)$. By assumption

$$
\partial \operatorname{Int} X^{d}(\Gamma)=\bigcup_{\Gamma_{1} \supset \Gamma} X\left(\Gamma ; \Gamma_{1}\right)
$$

where the right hand side are disjoint each other and $\Gamma_{1}$ are maximal. By induction hypothesis we have defined $s_{\epsilon}^{\Gamma_{1}}$ already. We now apply Lemma 9.1 to

$$
\pi_{\Gamma}:\left(s_{\epsilon}^{\Gamma_{1}}\right)^{-1}(0) \cap X\left(\Gamma ; \Gamma_{1}\right) \rightarrow\left(s_{\epsilon}^{\Gamma_{1}}\right)^{-1}(0) \cap X\left(\Gamma_{1}\right)
$$

and the bundle $E(\Gamma) \rightarrow\left(s_{\epsilon}^{\Gamma_{1}}\right)^{-1}(0) \cap X\left(\Gamma ; \Gamma_{1}\right)$. We then obtain $s_{\epsilon}^{\Gamma}$ on $\left(s_{\epsilon}^{\Gamma_{1}}\right)^{-1}(0) \cap$ $X\left(\Gamma ; \Gamma_{1}\right)$. We extend it to $X\left(\Gamma ; \Gamma_{1}\right) \backslash\left(s_{\epsilon}^{\Gamma_{1}}\right)^{-1}(0)$ in an arbitrary way. (It does not matter how we extend since it will not change the zero set.)

We have thus defined $s_{\epsilon}=s_{\epsilon}^{\Gamma_{1}} \oplus s_{\epsilon}^{\Gamma}$ on (43). Note that on

$$
\operatorname{Int} X^{d}(\Gamma) \backslash \partial \operatorname{Int} X^{d}(\Gamma)
$$

$E$ is decomposed to $E^{\Gamma}=E(\Gamma)$ and $E^{\perp}$. (We remark that we decompose $E^{\Gamma}$ to $E\left(\Gamma_{1}\right) \oplus E(\Gamma)$ only at their boundaries.) On the boundary we defined the section of $E^{\Gamma} \cong E(\Gamma) \oplus E\left(\Gamma_{1}\right)$ already which is of general position relative to zero. We can then extend it to $\operatorname{Int} X^{d}(\Gamma)$ so that it is of general position to zero. (Note $E^{\perp}$ component is necessarily zero again.) We then extend this to its neighborhood so that it is covariantly constant in each of $\pi_{\Gamma}$ fibers.

Now the main induction step goes as follows: Assuming $s_{\epsilon}^{\Gamma^{\prime}}$ is defined for $\# \Gamma^{\prime}>\# \Gamma$, we consider $\Gamma$. Take the decomposition

$$
\partial \operatorname{Int} X^{d}(\Gamma)=\bigcup X\left(\Gamma ; \Gamma_{1}, \Gamma_{2}, \ldots, \Gamma_{k}\right) .
$$

We will define $s_{\epsilon}^{\Gamma}$ on $X\left(\Gamma ; \Gamma_{1}, \Gamma_{2}, \ldots, \Gamma_{k}\right)$ by a downward induction on $k$.

We consider a chain of isotropy groups $\Gamma_{1}, \ldots, \Gamma_{k}$ given as in (39) for which $X\left(\Gamma ; \Gamma_{1}, \Gamma_{2}, \ldots, \Gamma_{k}\right)$ is nonempty. Let $k$ be maximal among such choices. We 
now apply Lemma 9.1 to

$$
\begin{aligned}
\pi_{\Gamma_{k}} & :\left(s_{\epsilon}^{\Gamma_{1}} \oplus \cdots \oplus s_{\epsilon}^{\Gamma_{k}}\right)^{-1}(0) \cap X\left(\Gamma ; \Gamma_{1}, \Gamma_{2}, \ldots, \Gamma_{k}\right) \\
& \rightarrow\left(s_{\epsilon}^{\Gamma_{1}} \oplus \cdots \oplus s_{\epsilon}^{\Gamma_{k}}\right)^{-1}(0) \cap X\left(\Gamma_{k} ; \Gamma_{1}, \Gamma_{2}, \ldots, \Gamma_{k-1}\right),
\end{aligned}
$$

and $E(\Gamma) \rightarrow X\left(\Gamma ; \Gamma_{1}, \Gamma_{2}, \ldots, \Gamma_{k}\right)$. Here we note that the well-definedness of (45) is a consequence of compatibilities of $\pi$ and $r$ stated in Definitions 8.3 and 8.4.

We thus obtain $s_{\epsilon}^{\Gamma}$ on $\left(s_{\epsilon}^{\Gamma_{1}} \oplus \cdots \oplus s_{\epsilon}^{\Gamma_{k}}\right)^{-1}(0) \cap X\left(\Gamma ; \Gamma_{1}, \Gamma_{2}, \ldots, \Gamma_{k}\right)$ which we extend to $X\left(\Gamma ; \Gamma_{1}, \Gamma_{2}, \ldots, \Gamma_{k}\right)$ in an arbitrary way.

Now we can extend $s_{\epsilon}^{\Gamma}$ to various $X\left(\Gamma ; \Gamma_{1}, \Gamma_{2}, \ldots, \Gamma_{\ell}\right)$ by a downward induction on $\ell$ using an appropriate relative version of Lemma 9.1. Namely we assume $s_{\epsilon}^{\Gamma}$ is defined on $X\left(\Gamma ; \Gamma_{1}, \Gamma_{2}, \ldots, \Gamma_{k}\right)$ for $k>\ell$ then $s_{\epsilon}^{\Gamma}$ is defined on $\partial X\left(\Gamma ; \Gamma_{1}, \Gamma_{2}, \ldots, \Gamma_{\ell}\right)$. Then we extend it to $X\left(\Gamma ; \Gamma_{1}, \Gamma_{2}, \ldots, \Gamma_{\ell}\right)$ by applying a relative version of Lemma 9.1 to

$$
\begin{aligned}
& \left(s_{\epsilon}^{\Gamma_{1}} \oplus \cdots \oplus s_{\epsilon}^{\Gamma_{\ell}}\right)^{-1}(0) \cap X\left(\Gamma ; \Gamma_{1}, \Gamma_{2}, \ldots, \Gamma_{\ell}\right) \\
& \rightarrow\left(s_{\epsilon}^{\Gamma_{1}} \oplus \cdots \oplus s_{\epsilon}^{\Gamma_{\ell}}\right)^{-1}(0) \cap X\left(\Gamma_{\ell} ; \Gamma_{1}, \Gamma_{2}, \ldots, \Gamma_{\ell-1}\right)
\end{aligned}
$$

and a bundle $E(\Gamma)$.

Thus we have constructed $s_{\epsilon}^{\Gamma}$ on (44). Again we extend $s_{\epsilon}^{\Gamma}$ to $\operatorname{Int} X^{d}(\Gamma)$ so that it is of general position to 0 .

Therefore we have constructed $s_{\epsilon}$ on the union

$$
\bigcup_{\Gamma} \operatorname{Int} X^{d}(\Gamma)
$$

We will extend this to $X$ as follows: We first observe

$$
X \backslash \bigcup_{\Gamma} \operatorname{Int} X^{d}(\Gamma)=\bigcup_{\Gamma}\left(\left(U^{\cong}(\Gamma)(d) \cap \pi_{\Gamma}^{-1}\left(\operatorname{Int} X^{d}(\Gamma)\right)\right) \backslash X^{\cong}(\Gamma)\right) .
$$

We will define $s_{\epsilon}$ on

$$
\left(\left(U^{\cong}(\Gamma)(d) \cap \pi_{\Gamma}^{-1}\left(\operatorname{Int} X^{d}(\Gamma)\right)\right) \backslash X^{\cong}(\Gamma)\right)
$$

by an upward induction on $\# \Gamma$. Let

$$
p \in\left(U^{\cong}(\Gamma)(d) \cap \pi_{\Gamma}^{-1}\left(\operatorname{Int} X^{d}(\Gamma)\right)\right) \backslash X^{\cong}(\Gamma)
$$

and consider

$$
r_{\Gamma}(d)(p) \in S^{\Gamma}(d) \subseteq \bigcup_{\Gamma^{\prime} \subsetneq \Gamma} U^{\cong}\left(\Gamma^{\prime}\right)(d) \cap \pi_{\Gamma^{\prime}}^{-1}\left(\operatorname{Int} X^{d}\left(\Gamma^{\prime}\right)\right)
$$


and

$$
\pi_{\Gamma}(p) \in \operatorname{Int} X^{d}(\Gamma) .
$$

We note that the right hand side of (47) contains the case $\Gamma^{\prime}=\{1\}$. In that case $U^{\cong}\left(\Gamma^{\prime}\right)(d) \cap \pi_{\Gamma^{\prime}}^{-1}\left(\operatorname{Int} X^{d}\left(\Gamma^{\prime}\right)\right)=\operatorname{Int} X^{d}\left(\Gamma^{\prime}\right)$.

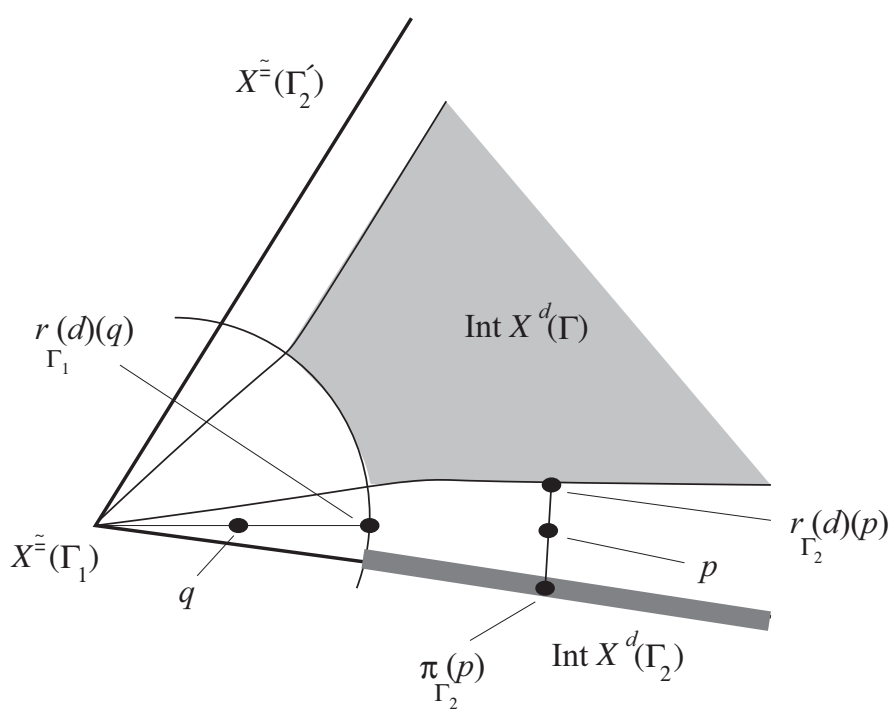

Figure 4

By construction, $s_{\epsilon}^{\Gamma}\left(\pi_{\Gamma}(p)\right)$ coincides with $s_{\epsilon}^{\Gamma}\left(r_{\Gamma}(d)(p)\right)$ under the identification

$$
E_{\pi_{\Gamma}(p)}(\Gamma) \cong E_{r_{\Gamma}(d)(p)}(\Gamma)
$$

We now put

$$
s_{\epsilon}(p)=s_{\epsilon}^{\Gamma}\left(\pi_{\Gamma}(p)\right)+\exp \left(\frac{1}{d^{2}}-\frac{1}{\rho_{\Gamma}(p)}\right) \sum_{\Gamma^{\prime} \subsetneq \Gamma} s_{\epsilon}^{\Gamma^{\prime}}\left(r_{\Gamma}(d)(p)\right) .
$$

This section coincides with previously defined one when $\sqrt{\rho_{\Gamma}(p)}=0$ or $d$. (We set $\exp \left(1 / d^{2}-1 / 0\right)=0$ as definition.) Hence it defines a piecewise smooth section on $X$.

We remark that by definition

$$
s_{\epsilon}^{-1}(0) \cap\left(U^{\cong}(\Gamma)(d) \cap \pi_{\Gamma}^{-1}\left(\operatorname{Int} X^{d}(\Gamma)\right)\right)
$$

is the cone of the map

$$
\pi_{\Gamma}: s_{\epsilon}^{-1}(0) \cap S^{\Gamma}(d) \rightarrow X^{\cong}(\Gamma) .
$$


Since we constructed our section applying Lemma 9.1 repeatedly, it follows that this cone has a smooth triangulation. (We use Definition 8.4 (vii) and the argument of section 3,4 [16] for this.) On (46) we have a transversality and hence Proposition 7.1 (ii) holds. Proposition 7.1 (iii) and Proposition 7.1 (iv) are also obvious from construction. The proof of Proposition 7.1 is now complete.

\section{Single valued perturbation of a space with Kuranishi STRUCTURE: PROOF OF THEOREM 3.1}

In this section we prove Theorem 3.1. For the proof we need the following relative version of Proposition 7.1.

Definition 10.1. A piecewise smooth single-valued section of an orbi-bundle $E / G \rightarrow X=M / G$ is said to be normally conical if the following holds:

(i) There is a decomposition of $X=M / G$ into

$$
\bigcup_{\Gamma} \operatorname{Int} X^{d}(\Gamma) \cup \bigcup_{\Gamma}\left(X^{\cong}(\Gamma) \backslash \operatorname{Int} X^{d}(\Gamma)\right)
$$

as in $(36)$.

(ii) On $\operatorname{Int} X^{d}(\Gamma)$ the $E^{\perp}$-component $s$ is of general position to 0 . (The $E^{\Gamma}$ component is necessarily 0 .)

(iii) On $X^{\cong}(\Gamma) \backslash \operatorname{Int} X^{d}(\Gamma)$, the section $s$ is given by (48).

Proposition 10.1. Let $X$ be a global quotient, $K$ a compact subset of $X$ and $U$ a neighborhood of $K$. Let $s$ be a $C^{0}$-section of the orbi-bundle $E / G \rightarrow X$. We assume that $s$ satisfies Proposition 7.1 (i)-(iv) on $U$ and is normally conical in the above sense. Then there exists a sequence of single-valued piecewise smooth sections $s_{\epsilon}$ converging to $s$ in $C^{0}$ sense satisfying Proposition 7.1 (i)-(iv) such that $s_{\epsilon}=s$ on $K$.

The proof is the same as Proposition 7.1 and is omitted.

Proof of Theorem 3.1. The proof is by induction on $p \in P$ with respect to the partial order $<$. (See Lemma 2.1 for the finite set $P$ and the partial order.) If $p$ is minimal, we apply Proposition 7.1 to obtain $s_{p}^{\prime}$. Assume that we have $s_{q}^{\prime}$ for every $q<p$. We consider $s_{q}^{\prime}$ and the image $\phi_{p q}\left(V_{p q}\right)$. We restrict $s_{q}^{\prime}$ on the image $\phi_{p q}\left(V_{p q}\right)$ and use the embedding $\hat{\phi}_{p q}$ to obtain a section of $\left.E_{q}\right|_{\phi_{p q}\left(V_{p q}\right)} \rightarrow V_{p q}$. 
We can extend it to its neighborhood, so that the compatibility in the sense of Definition 2.7 is satisfied.

We note that the required properties (i) - (ii) are satisfied on the tubular neighborhood $N_{\phi_{p q}\left(V_{p q}\right)}$ if it is satisfied by $s_{q}^{\prime}$.

Now we can use Propositions 10.1 to obtain the section $s_{p}^{\prime}$. The proof of Theorem 3.1 is complete.

\section{Moduli SPACE OF PSEUdo-HOlOMORPhiC DisCs: ReVIEW}

We will apply Theorem 3.1 to the moduli space of pseudo-holomorphic discs. We review the definition and basic facts on the moduli space in this section.

Let $(M, \omega)$ be a symplectic manifold and $L$ a Lagrangian submanifold of $M$. We take a compatible almost complex structure $J$ on $M$. Let $\beta \in H_{2}(M, L ; \mathbb{Z})$. We consider $\left(v ; z_{0}, \ldots, z_{k}\right)$ such that

(1) $v:\left(D^{2}, \partial D^{2}\right) \rightarrow(M, L)$ is $J$-holomorphic.

(2) $z_{i} \in \partial D^{2} . z_{i} \neq z_{j}$ for $i \neq j$.

(3) $\left(z_{0}, \ldots, z_{k}\right)$ respects the counter clock-wise cyclic order of $\partial D^{2}$.

(4) The homology class $v_{*}\left(\left[D^{2}\right]\right) \in H_{2}(M, L)$ is $\beta$.

We say $\left(v ; z_{0}, \ldots, z_{k}\right)$ and $\left(v^{\prime} ; z_{0}^{\prime}, \ldots, z_{k}^{\prime}\right)$ are equivalent if there exists a biholomorphic map $\varphi: D^{2} \rightarrow D^{2}$ such that $\varphi\left(z_{i}^{\prime}\right)=z_{i}$ and $v \circ \varphi=v^{\prime}$. The set of the equivalence classes is written as $\mathcal{M}_{k+1}^{\text {main,reg }}(\beta)$. We can compactify it as follows. We consider a pair $((\Sigma, \vec{z}), v)$ where $v: \Sigma \rightarrow M, \Sigma$ is a bordered Riemann surface of genus zero, $\vec{z}=\left(z_{0}, \ldots, z_{k}\right)$ and $z_{i} \in \partial \Sigma$ satisfies (2) and (3) above. We also assume $v$ is a $J$-holomorphic map of homology class $\beta$.

Moreover we assume that $((\Sigma, \vec{z}), v)$ is stable: namely there exist only a finite number of biholomorphic maps $\varphi: \Sigma \rightarrow \Sigma$ such that $\varphi\left(z_{i}\right)=z_{i}$ and $v \circ \varphi=v$. We say $((\Sigma, \vec{z}), v) \sim\left(\left(\Sigma^{\prime}, \vec{z}^{\prime}\right), v^{\prime}\right)$ if there exists a biholomorphic map $\varphi: \Sigma^{\prime} \rightarrow \Sigma$ such that $\varphi\left(z_{i}^{\prime}\right)=z_{i}$ and $v \circ \varphi=v^{\prime}$. We denote by $\mathcal{M}_{k+1}^{\text {main }}(\beta)$ the set of $\sim$ equivalence classes of such $\left(\left(\Sigma,\left(z_{0}, \ldots, z_{k}\right)\right), v\right)$. (See Subsection 2.1.2 [6] for detail.)

We define a map $e v=\left(e v_{0}, \ldots, e v_{k}\right): \mathcal{M}_{k+1}^{\text {main }}(\beta) \rightarrow L^{k}$ by

$$
e v_{i}\left(\left(\Sigma,\left(z_{0}, \ldots, z_{k}\right)\right), v\right)=v\left(z_{i}\right)
$$


Theorem 11.1. We can define a topology on $\mathcal{M}_{k+1}^{\operatorname{main}}(\beta)$ with respect to which it is compact and Hausdorff.

$\mathcal{M}_{k+1}^{\text {main }}(\beta)$ has a Kuranishi structure with corner. Its (virtual) dimension is given by:

$$
\operatorname{dim} \mathcal{M}_{k+1}^{\operatorname{main}}(\beta)=n+\mu_{L}(\beta)+k-2 .
$$

Here $\mu_{L}(\beta)$ is the Maslov index of $\beta$. Moreover $\mathcal{M}_{k+1}^{\operatorname{main}}(\beta)$ has a tangent space. If $L$ is relatively spin, $\mathcal{M}_{k+1}^{\text {main }}(\beta)$ is orientable. The orientation is determined by the relative spin structure.

The evaluation map ev induces a strongly smooth and weakly submersive map.

This theorem is proved in Section $7.1[7]$.

Let $P_{i}(i=1, \ldots, k)$ be simplices and $f_{i}: P_{i} \rightarrow L$ smooth maps. We define:

$$
\mathcal{M}_{k+1}^{\text {main }}\left(\beta ; P_{1}, \ldots, P_{k}\right)=\mathcal{M}_{k+1}^{\text {main }}(\beta)_{\left(e v_{1}, \ldots, e v_{k}\right)} \times_{\left(f_{1}, \ldots, f_{k}\right)}\left(P_{1} \times \cdots \times P_{k}\right) .
$$

(Here and hereafter we write $P_{i}$ in place of the singular chain $\left(P_{i}, f_{i}\right)$.) Since $e v$ is weakly submersive, we can use Lemma A1.39 [7] to define a Kuranishi structure on $\mathcal{M}_{k+1}^{\text {main }}\left(\beta ; P_{1}, \ldots, P_{k}\right)$. We will define the filtered $A_{\infty}$ structure $\mathfrak{m}_{k}$ by

$$
\mathfrak{m}_{k}\left(P_{1}, \ldots, P_{k}\right)=\sum_{\beta} T^{\omega[\beta]} e^{\mu_{L}(\beta) / 2} e v_{0 *}\left(\left[\mathcal{M}_{k+1}^{\operatorname{main}}\left(\beta ; P_{1}, \ldots, P_{k}\right)\right]\right) .
$$

Here $e v_{0 *}\left(\left[\mathcal{M}_{k+1}^{\operatorname{main}}\left(\beta ; P_{1}, \ldots, P_{k}\right)\right]\right)$ is the virtual fundamental chain of this moduli space, which is regarded as a chain on $L$ by the map $e v_{0}$. In general, virtual fundamental chain depends on the choice of perturbation.

In [6], [7] we used multivalued perturbations (or multisections) to define this virtual fundamental chain. So it is defined over $\mathbb{Q}$. (We need the orientation, which is induced by the relative spin structure.) In this paper we will use Theorem 3.1 to define a virtual fundamental chain over $\mathbb{Z}$ or $\mathbb{Z}_{2}$.

In the next section we will also use a moduli space $\mathcal{M}_{k+1, \ell}^{\text {main }}\left(\beta ; P_{1}, \ldots, P_{k}\right)$ which is defined as follows: We consider $((\Sigma, \vec{z}, \vec{w}), v)$, where $((\Sigma, \vec{z}), v)$ satisfies the same assumption as in the definition of $\mathcal{M}_{k+1}^{\text {main }}\left(\beta ; P_{1}, \ldots, P_{k}\right)$, except the stability, $\vec{w}=\left(w_{1}, \ldots, w_{\ell}\right), w_{i} \in \Sigma \backslash \partial \Sigma$, and $w_{i} \neq w_{j}$ for $i \neq j$. We assume $((\Sigma, \vec{z}, \vec{w}), v)$ is stable: namely there exists only a finite number of biholomorphic maps $\varphi: \Sigma \rightarrow \Sigma$ such that $\varphi\left(z_{i}\right)=z_{i}, \varphi\left(w_{i}\right)=w_{i}$ and $v \circ \varphi=v$. We define an equivalence relation 
on the set of such $((\Sigma, \vec{z}, \vec{w}), v)$ in a similar way. The set of equivalence classes is denoted by $\mathcal{M}_{k+1, \ell}^{\text {main }}\left(\beta ; P_{1}, \ldots, P_{k}\right)$.

We can generalize Theorem 11.1 to $\mathcal{M}_{k+1, \ell}^{\text {main }}\left(\beta ; P_{1}, \ldots, P_{k}\right)$, where the formula (49) is replaced by

$$
\operatorname{dim} \mathcal{M}_{k+1, \ell}^{\operatorname{main}}(\beta)=n+\mu_{L}(\beta)+2 \ell+k-2 .
$$

Using the evaluation map $e v=\left(e v_{0}, \ldots, e v_{k}\right): \mathcal{M}_{k+1, \ell}^{\text {main }}(\beta) \rightarrow L^{k+1}$ we define the fiber product $\mathcal{M}_{k+1, \ell}^{\text {main }}\left(\beta ; P_{1}, \ldots, P_{k}\right)$ in the same way as in (50).

\section{Estimate of the Virtual Dimension of the Singular LOCUS}

The main result of this section is:

Proposition 12.1. Suppose $(M, \omega, J)$ is spherically positive. Then for each $\Gamma \neq$ $\{1\}$ and $i$, we have

$$
\operatorname{dim} \mathcal{M}_{k+1}^{\operatorname{main}}\left(\beta ; P_{1}, \ldots, P_{k}\right)-d\left(\mathcal{M}_{k+1}^{\operatorname{main}}\left(\beta ; P_{1}, \ldots, P_{k}\right) ; \Gamma ; i\right) \geq 2 .
$$

We recall that $d\left(\mathcal{M}_{k+1}^{\operatorname{main}}\left(\beta ; P_{1}, \ldots, P_{k}\right) ; \Gamma ; i\right)$ is defined in $(29)$.

Proof. Let $((\Sigma, \vec{z}), v, \vec{x})$ be an element of $\mathcal{M}_{k+1}^{\text {main }}\left(\beta ; P_{1}, \ldots, P_{k}\right)$ : Namely $\Sigma$ is a genus zero bordered Riemann surface, $v:(\Sigma, \partial \Sigma) \rightarrow(M, L)$ is pseudoholomorphic, $\vec{z}=\left(z_{0}, \ldots, z_{k}\right)$ are boundary marked points of $\Sigma$, and

$$
\vec{x}=\left(x_{1}, \ldots, x_{k}\right), \quad x_{i} \in P_{i} \quad \text { satisfying } v\left(z_{i}\right)=f_{i}\left(x_{i}\right) .
$$

Here $f_{i}: P_{i} \rightarrow L$ and $\left(P_{i}, f_{i}\right)$ are regarded as smooth singular chains of $L$, which we write just as $P_{i}$ by an abuse of notation.

We recall that the genus of $\Sigma$ is zero and the disc components cannot have non-trivial automorphism groups since it has at least one special point, i.e., either marked or nodal points, on the boundary. Therefore for every non-trivial element $\varphi \in \operatorname{Aut}((\Sigma, \vec{z}), v)$ and any sphere component $S_{i}^{2} \cong S^{2} \subset \Sigma$ preserved by $\varphi$, the automorphism $\varphi$ acts as the multiplication by $e^{2 \pi \sqrt{-1} \ell / k}$,

$$
z \mapsto e^{2 \pi \sqrt{-1} \ell / k} z
$$

with the identification $S_{i}^{2}=\mathbb{C} \cup\{\infty\}$. And $\varphi$ permutes other components: This is because any finite subgroup of $\operatorname{PSL}(2 ; \mathbb{C})=\operatorname{Aut}\left(S^{2}\right)$ that fixes $\infty$ is conjugate to such a group. 
As a consequence, the quotient space $(\bar{\Sigma}, \vec{z})=\Sigma / \operatorname{Aut}((\Sigma, \vec{z}), v)$ is again a (pointed) bordered Riemann surface of genus zero. The pseudo-holomorphic map $v$ induces a map $\bar{v}: \Sigma / \operatorname{Aut}((\Sigma, \vec{z}), v) \rightarrow M$.

Definition 12.1. We call $((\bar{\Sigma}, \vec{z}), \bar{v}, \vec{x})$ the reduced model of $((\Sigma, \vec{z}), v, \vec{x})$.

We note that the reduced model could be unstable. Namely there may appear a sphere component with two singular points where the map $\bar{v}$ is trivial. Even if the reduced model is stable, it may have a nontrivial automorphism.

Remark 12.2. We note that the notions of "trivial automorphism" and "somewhere injective" are two different notions: Somewhere injectivity implies triviality of the automorphism group, but not the other way around. For example, there is a branched covering $S^{2} \rightarrow S^{2}$ with no nontrivial automorphism. For the abstract perturbation $\mathfrak{s}^{\epsilon}$, its transversality to zero is related to the existence of nontrivial automorphism but not to the somewhere injectivity. (Somewhere injectivity is essential if one uses perturbations only of $J$ to achieve transversality.)

We now compare the virtual dimension of $((\Sigma, \vec{z}), v, \vec{x}) \in \mathcal{M}_{k+1}^{\operatorname{main}}\left(\beta ; P_{1}, \ldots, P_{k}\right)$ with that of its reduced model. We begin with a discussion of the deformation complex of a multiple sphere. Let $\alpha \in \pi_{2}(M)$ and $\widetilde{\mathcal{M}}^{\text {reg }}(M ; \alpha)$ be the set of pseudo-holomorphic maps $u: S^{2} \rightarrow M$ with $[u]=\alpha$. For $u \in \widetilde{\mathcal{M}}^{\text {reg }}(M ; \alpha)$ we define $\mathfrak{R}_{m}(u) \in \widetilde{\mathcal{M}}^{\mathrm{reg}}(M ; m \alpha)$ by $\mathfrak{R}_{m}(u)(z)=u\left(z^{m}\right)$.

For each $v \in \widetilde{\mathcal{M}}^{\text {reg }}(M ; m \alpha)$, we consider the linearization

$$
D_{v} \bar{\partial}: \Gamma\left(S^{2} ; v^{*} T M\right) \rightarrow \Gamma\left(S^{2} ; \Lambda^{0,1}\left(v^{*} T M\right)\right)
$$

of the Cauchy-Riemann section $\bar{\partial}$. We denote by $C(v)=\left(C_{0}(v), C_{1}(v), D_{v} \bar{\partial}\right)$ the elliptic complex given by

$$
C_{0}(v)=\Gamma\left(S^{2} ; v^{*} T M\right), \quad C_{1}(v)=\Gamma\left(S^{2} ; \Lambda^{0,1}\left(v^{*} T M\right)\right) .
$$

We consider the assignment of the pull-back complex $C\left(\Re_{m}(u)\right)$ to $u \in$ $\widetilde{\mathcal{M}}^{\text {reg }}(M ; \alpha)$ on which the group $\mathbb{Z}_{m}$ acts. We regard this assignment of $\mathbb{Z}_{m^{-}}$ modules as a $\mathbb{Z}_{m}$-equivariant family index over $\widetilde{\mathcal{M}}^{\text {reg }}(M ; \alpha)$.

Lemma 12.2. The index of $C\left(\Re_{m}(u)\right)$ as a $\mathbb{Z}_{m}$-module is

$$
2 c_{1}(M)(\alpha) \operatorname{Reg}_{\mathbb{Z}_{m}} \oplus 2 n \mathbf{1} .
$$

Here $\operatorname{Reg}_{\mathbb{Z}_{m}}$ is the regular representation of $\mathbb{Z}_{m}$ and $\mathbf{1}$ is its trivial representation. 
Proof. Let $\gamma$ be an element of $\mathbb{Z}_{m}$ with $\gamma \neq$ unit. We use the Lefschetz fixed point formula by Atiyah-Bott [1] to obtain

$$
\sum_{*=0,1}(-1)^{*} \operatorname{Tr}\left(\gamma: H^{*}\left(C\left(\mathfrak{R}_{m}(u)\right) \rightarrow H^{*}\left(C\left(\Re_{m}(u)\right)\right)=2 n .\right.\right.
$$

Note there are only two fixed points of $\gamma$ and we take the trace over $\mathbb{R}$. On the other hand, the numerical index of $D_{\mathfrak{R}_{m}(u)} \bar{\partial}$ is $2 n+2 m c_{1}(M)(\alpha)$, which coincides with the super-trace of the unit element $e \in \mathbb{Z}_{m}$. The lemma follows immediately.

(We note that we can also prove Lemma 12.2 by directly calculating the kernel and cokernel without using [1].)

In particular, Lemma 12.2 implies that the $\mathbb{Z}_{m}$-invariant part of the index of $C\left(\Re_{m}(u)\right)$ for the $\mathbb{Z}_{m}$-cover of a holomorphic sphere is equal to the index of $C(u)$ for its reduced model. We will use this fact in the proof of Proposition 12.3 coming later.

Let $((\Sigma, \vec{z}), v, \vec{x}) \in \mathcal{M}_{k+1}^{\text {main }}\left(\beta: P_{1}, \ldots, P_{k}\right)$ and $((\bar{\Sigma}, \vec{z}), \bar{v}, \vec{x})$ be its reduced model. We first recall the definition of the deformation complex of $((\Sigma, \vec{z}), v, \vec{x})$ which is an elliptic complex acted upon by the group $\Gamma$ of automorphisms of $((\Sigma, \vec{z}), v)$.

We decompose $\Sigma$ into irreducible components $\Sigma=\bigcup_{a} \Sigma_{a}$ where $\Sigma_{a}$ is a sphere or a disc and put $v_{a}=\left.v\right|_{\Sigma_{a}}$ and consider the elliptic complex

$$
C\left(v_{a}\right)=\left(C_{0}\left(v_{a}\right), C_{1}\left(v_{a}\right), D_{v_{a}} \bar{\partial}\right)
$$

where

$$
D_{v_{a}} \bar{\partial}: C_{0}\left(v_{a}\right)=\Gamma\left(\Sigma_{a}, \partial \Sigma_{a} ; v_{a}^{*} T M, v_{a}^{*} T L\right) \rightarrow C_{1}\left(v_{a}\right)=\Gamma\left(\Sigma_{a} ; \Lambda^{0,1}\left(v_{a}^{*} T M\right)\right) .
$$

(The boundary condition $v_{a}^{*} T L$ is empty if $\Sigma_{a}=S^{2}$.) For each singular point $z_{i}^{\text {sing }}$ we take $z_{i, 1}^{\text {sing }} \in \Sigma_{a(i, 1)}, z_{i, 2}^{\text {sing }} \in \Sigma_{a(i, 2)}$ which are $z_{i}^{\text {sing }}$ in $\Sigma$. We put

$$
\left\{\begin{array}{l}
\widetilde{C}_{0}((\Sigma, \vec{z}), v)^{+}=\bigoplus_{a} C_{0}\left(v_{a}\right), \\
\widetilde{C}_{0}((\Sigma, \vec{z}), v)=\left\{\left(W_{a}\right) \in \bigoplus_{a} C_{0}\left(v_{a}\right) \mid W_{a(i, 1)}\left(z_{i, 1}^{\text {sing }}\right)=W_{a(i, 2)}\left(z_{i, 2}^{\text {sing }}\right)\right\} .
\end{array}\right.
$$

We put $\widetilde{C}_{1}((\Sigma, \vec{z}), v)=\bigoplus_{a} C_{1}\left(v_{a}\right)$. The operators $D_{v_{a}} \bar{\partial}$ induce

$$
D_{v} \bar{\partial}: \widetilde{C}_{0}((\Sigma, \vec{z}), v) \rightarrow \widetilde{C}_{1}((\Sigma, \vec{z}), v)
$$


Let $\operatorname{Aut}(\Sigma, \vec{z})$ be the group of all automorphisms of $(\Sigma, \vec{z})$. We have a canonical homomorphism of its Lie algebra aut $(\Sigma, \vec{z})$ into $\widetilde{C}_{0}((\Sigma, \vec{z}), v)$ : Note that by the definition of $\operatorname{Aut}(\Sigma, \vec{z})$ any element of $\operatorname{aut}(\Sigma, \vec{z})$ has its value zero at the singular points. The stability condition implies that this homomorphism is injective and so we may regard aut $(\Sigma, \vec{z})$ as a subspace of $\widetilde{C}_{0}((\Sigma, \vec{z}), v)$. Moreover the image of $\operatorname{aut}(\Sigma, \vec{z})$ lies in the kernel of $D_{v} \bar{\partial}$. Therefore we have the following complex

$$
0 \rightarrow \operatorname{aut}(\Sigma, \vec{z}) \rightarrow \widetilde{C}_{0}((\Sigma, \vec{z}), v) \rightarrow \widetilde{C}_{1}((\Sigma, \vec{z}), v) .
$$

We put

$$
\begin{gathered}
\widetilde{C}_{0}((\Sigma, \vec{z}), v, \vec{x})=\left\{\left(\left(W_{a}\right),\left(\mathrm{v}_{i}\right)\right) \mid\left(W_{a}\right) \in \widetilde{C}_{0}((\Sigma, \vec{z}), v), \mathrm{v}_{i} \in T_{x_{i}} P_{i},\right. \\
\left.W_{a_{i}}\left(z_{i}\right)=\left(d_{x_{i}} f_{i}\right)\left(\mathrm{v}_{i}\right)\right\} .
\end{gathered}
$$

(We note that $f_{i}: P_{i} \rightarrow L$ is our smooth singular simplex.) Since $\operatorname{Aut}(\Sigma, \vec{z})$ fixes the marked points $\vec{z}$, it induces an action on $\mathcal{M}_{k+1}^{\operatorname{main}}\left(\beta: P_{1}, \ldots, P_{k}\right)$ and so its Lie algebra aut $(\Sigma, \vec{z})$ injects to $\widetilde{C}_{0}((\Sigma, \vec{z}), v, \vec{x})$. This leads us to define

$$
\begin{aligned}
& C_{0}((\Sigma, \vec{z}), v, \vec{x}):=\widetilde{C}_{0}((\Sigma, \vec{z}), v, \vec{x}) / \operatorname{aut}(\Sigma, \vec{z}) \\
& C_{1}((\Sigma, \vec{z}), v, \vec{x}):=\widetilde{C}_{1}((\Sigma, \vec{z}), v) .
\end{aligned}
$$

Here $z_{i} \in \Sigma_{a_{i}}$. The operator $D_{v} \bar{\partial}$ also induces a homomorphism $C_{0}((\Sigma, \vec{z}), v, \vec{x}) \rightarrow$ $C_{1}((\Sigma, \vec{z}), v, \vec{x})$. We denote by

$$
C((\Sigma, \vec{z}), v, \vec{x}), \quad \widetilde{C}((\Sigma, \vec{z}), v, \vec{x})
$$

the complexes

$$
\begin{aligned}
& D_{v} \bar{\partial}: C_{0}((\Sigma, \vec{z}), v, \vec{x}) \rightarrow C_{1}((\Sigma, \vec{z}), v, \vec{x}), \\
& D_{v} \bar{\partial}: \widetilde{C}_{0}((\Sigma, \vec{z}), v, \vec{x}) \rightarrow \widetilde{C}_{1}((\Sigma, \vec{z}), v, \vec{x}),
\end{aligned}
$$

respectively. The group $\Gamma=\operatorname{Aut}(\Sigma, \vec{z})$ acts on these complexes in an obvious way.

To describe the relation between the deformation complex of $((\Sigma, \vec{z}), v, \vec{x})$ and that of its reduced model, we need one more notation.

Definition 12.3. We call a point $z \in \Sigma$ a free fixed point if the following holds:

(i) $z$ is not a singular point.

(ii) $z$ is on a sphere component $S_{a}^{2}$ of $\Sigma$ such that there exists $\gamma \in \Gamma$ which preserves $S_{a}^{2}$ and acts nontrivially on $S_{a}^{2}$. Moreover $\gamma(z)=z$. 
For $\Gamma^{\prime} \subset \Gamma$ we denote by $F\left(\Gamma^{\prime}\right)$ the set of all $z$ satisfying (i), (ii) above for $\gamma \in \Gamma^{\prime}$.

For $\Gamma^{\prime} \subset \Gamma$ we define

$$
C\left(F\left(\Gamma^{\prime}\right)\right)=\bigoplus_{z \in F\left(\Gamma^{\prime}\right)} \mathbb{C}[z]
$$

the vector space whose basis is identified with $F\left(\Gamma^{\prime}\right)$.

In case $\Gamma^{\prime \prime}$ normalizes a subgroup $\Gamma^{\prime}(\subset \Gamma), \Gamma^{\prime \prime}$ acts on $F\left(\Gamma^{\prime}\right)$ in an obvious way and so induces an action on $C\left(F\left(\Gamma^{\prime}\right)\right)$. We put

$$
C\left(F\left(\Gamma^{\prime}\right)\right)^{\Gamma^{\prime \prime}}=\left\{v \in C\left(F\left(\Gamma^{\prime}\right)\right) \mid \forall \gamma \in \Gamma^{\prime \prime}, \gamma v=v\right\} .
$$

It is easy to see that

$$
\operatorname{dim}_{\mathbb{C}} C\left(F\left(\Gamma^{\prime}\right)\right)^{\Gamma^{\prime \prime}}=\#\left(F\left(\Gamma^{\prime}\right) / \Gamma^{\prime \prime}\right)
$$

and in particular

$$
\operatorname{dim}_{\mathbb{C}} C(F(\Gamma))^{\Gamma}=\#(F(\Gamma) / \Gamma) .
$$

Definition 12.4. For each sphere component $S_{a}^{2}$, we define its distance from the disc components as the minimal edge distance of the vertex corresponding to the component $S_{a}^{2}$ from the vertices corresponding to the disc components in the dual graph of $\Sigma$.

We recall that the minimal edge distance between two vertices in a graph is defined to be the minimum number of edges in all connected paths between the two in the graph. We denote by $\Sigma_{d}$ the union of all disc components and the sphere components whose distance from the disc components are $\leq d$. By definition $\Sigma_{0}$ is the union of all the disc components.

Let $\left(S_{a}^{2}, \vec{p}_{a}, o_{a}\right)$ be a sphere component of $((\Sigma, \vec{z}), v)$, whose distance from the disc components is $d$. Here $o_{a}$ is the point where $S_{a}^{2}$ is attached to $\Sigma_{d-1}$ and $\vec{p}_{a}$ is the set of other singular points, i.e., those to which some sphere components of distance $d+1$ from the disc components are attached. Put $n_{a}=\# \vec{p}_{a}$ and let $\Gamma_{a}$ be the group of automorphisms on $\left(S_{a}^{2}, \vec{p}_{a}, o_{a}\right)$ consisting of the restrictions of some elements of $\Gamma$ to $S_{a}^{2}$. Denote by $\operatorname{Conf}_{m+1}\left(\mathbb{C} P^{1}\right)$ the moduli space (i.e., divided by the action of $\left.P S L_{2}(\mathbb{C})\right)$ of $m+1$ ordered points on $\mathbb{C} P^{1}$. Denote by Conf $_{m+1}^{\Gamma_{a}}\left(\mathbb{C} P^{1}\right)$ the moduli space of distinct $m+1$ points on $\mathbb{C} P^{1}$ with the symmetry group $\Gamma_{a}$, (that is the set of $\left(\mathbb{C} P^{1},\left(z_{0}, \ldots, z_{m}\right)\right)$ such that $\gamma z_{i} \in\left\{z_{0}, \ldots, z_{m}\right\}$ 
for each $i=1, \ldots, m$ and $\left.\gamma \in \Gamma_{a}.\right)$ Let $\operatorname{Aut}\left(S_{a}, \vec{p}_{a}, o_{a}\right)$ be the group of all biholomorphic maps $\varphi: S_{a} \rightarrow S_{a}$ such that $\varphi\left(\vec{p}_{a}\right)=\vec{p}_{a}$ and $\varphi\left(o_{a}\right)=o_{a}$. We put

$$
\operatorname{Aut}\left(S_{a}, \vec{p}_{a}, o_{a}\right)^{\Gamma_{a}}=\left\{\varphi \in \operatorname{Aut}\left(S_{a}, \vec{p}_{a}, o_{a}\right) \mid \forall \gamma \in \Gamma_{a}, \varphi \circ \gamma=\gamma \circ \varphi\right\}
$$

We define

$$
\rho^{\Gamma_{a}}\left(S_{a}, \vec{p}_{a}, o_{a}\right)= \begin{cases}-\operatorname{dim}_{\mathbb{R}} \operatorname{Aut}\left(S_{a}, \vec{p}_{a}, o_{a}\right)^{\Gamma_{a}} & \text { if } n_{a}+1<3 \\ \operatorname{dim}_{\mathbb{R}} \operatorname{Conf}_{n_{a}+1}^{\Gamma_{a}}\left(\mathbb{C} P^{1}\right) & \text { if } n_{a}+1 \geq 3 .\end{cases}
$$

Let $\left(\bar{S}_{b}, \overrightarrow{\bar{p}}_{b}, \bar{o}_{b}\right)$ be a sphere component of the reduced model $((\bar{\Sigma}, \overrightarrow{\bar{z}}), \bar{v})$. Here $\bar{o}_{b}$ is the point of $\bar{S}_{b}$ at which it is attached to $\bar{\Sigma}_{d-1}$, and $\vec{p}_{b}$ are those at which some sphere components of distance $d+1$ are attached. Put $\bar{n}_{b}=\# \overrightarrow{\bar{p}}_{b}$. We define

$$
\bar{\rho}\left(\bar{S}_{b}, \overrightarrow{\bar{p}}_{b}, \bar{o}_{b}\right)= \begin{cases}-\operatorname{dim}_{\mathbb{R}} \operatorname{Aut}\left(S_{b}, \overrightarrow{\bar{p}}_{b}, \bar{o}_{b}\right) & \text { if } \bar{n}_{b}+1<3 \\ \operatorname{dim}_{\mathbb{R}} \operatorname{Conf}_{\bar{n}_{b}+1}\left(\mathbb{C} P^{1}\right) & \text { if } \bar{n}_{b}+1 \geq 3 .\end{cases}
$$

Let $\left(D_{a}^{2} ; \vec{p}_{a}^{D}, \vec{p}_{a}^{S}\right)$ be a disc component of $\Sigma$. Here $\vec{p}_{a}^{D}=\left(p_{a, 1}^{D}, \ldots, p_{a, m_{a}}^{D}\right)$ is the boundary marked points and $\vec{p}_{a}^{S}=\left(p_{a, 1}^{S}, \ldots, p_{a, n_{a}}^{S}\right)$ is the interior marked points. We put

$$
\rho\left(D_{a}^{2} ; \vec{p}_{a}^{D}, \vec{p}_{a}^{S}\right)=m_{a}+2 n_{a}-3 .
$$

This number is the negative of the dimension of the automorphism group if $m_{a}+2 n_{a} \leq 3$ and is the dimension of appropriate moduli space (of $\mathbb{C} P^{1}$ with marked points) if $m_{a}+2 n_{a} \geq 3$. (We remark that the $\Gamma$-action is trivial on each disc component.) We define $\bar{\rho}\left(\bar{D}_{b}^{2} ; \overrightarrow{\bar{p}}_{b}^{D}, \overrightarrow{\bar{p}}_{b}^{S}\right)$ by the same formula for the disc component $\left(\bar{D}_{b}^{2} ; \overrightarrow{\bar{p}}_{b}^{D}, \overrightarrow{\bar{p}}_{b}^{S}\right)$ of $\bar{\Sigma}$.

Let $\left\{S_{a(b)} \mid b \in \bar{I}\right\}$ be a complete set of representatives of the $\Gamma$-orbits in the set of sphere components of $((\Sigma, \vec{z}), v)$. Let $I^{D}$ and $\bar{I}^{D}$ be the sets of disc components of $\Sigma$ and $\bar{\Sigma}$, respectively. Note that there is a canonical identification between the two sets. We define

$$
\rho^{\Gamma}((\Sigma, \vec{z}), v)=\sum_{b \in \bar{I}} \rho^{\Gamma_{a(b)}}\left(S_{a(b)}, \vec{p}_{a(b)}, o_{a(b)}\right)+\sum_{a \in I^{D}} \rho\left(D_{a}^{2} ; \vec{p}_{a}^{D}, \vec{p}_{a}^{S}\right) .
$$

For the reduced model, we define

$$
\bar{\rho}((\bar{\Sigma}, \overrightarrow{\bar{z}}), \bar{v})=\sum_{b \in \bar{I}} \rho\left(\bar{S}_{b}, \overrightarrow{\bar{p}}_{b}, \bar{o}_{b}\right)+\sum_{b \in \bar{I}^{D}} \bar{\rho}\left(\bar{D}_{b}^{2} ; \overrightarrow{\bar{p}}_{b}^{D}, \overrightarrow{\bar{p}}_{b}^{S}\right) .
$$

Then we have the following: 
Proposition 12.3.

$$
\begin{aligned}
& \operatorname{dim}_{\mathbb{R}} \operatorname{Index}(\widetilde{C}((\Sigma, \vec{z}), v, \vec{x}))^{\Gamma}+\rho^{\Gamma}((\Sigma, \vec{z}), v) \\
& =\operatorname{dim}_{\mathbb{R}} \operatorname{Index}(\widetilde{C}((\bar{\Sigma}, \overrightarrow{\bar{z}}), \bar{v}, \vec{x}))+\bar{\rho}((\bar{\Sigma}, \overrightarrow{\vec{z}}), \bar{v})+\operatorname{dim}_{\mathbb{R}} C(F(\Gamma))^{\Gamma} .
\end{aligned}
$$

Proof. We begin with the following lemma.

\section{Lemma 12.4.}

$$
\operatorname{dim}_{\mathbb{R}} \operatorname{Index}(\widetilde{C}((\Sigma, \vec{z}), v, \vec{x}))^{\Gamma}=\operatorname{dim}_{\mathbb{R}} \operatorname{Index}(\widetilde{C}((\bar{\Sigma}, \overrightarrow{\bar{z}}), \bar{v}, \vec{x})) .
$$

Proof. Let us consider the complex $\widetilde{C}((\Sigma, \vec{z}), v, \vec{x})^{+}$

$$
D_{v} \bar{\partial}: \widetilde{C}_{0}((\Sigma, \vec{z}), v, \vec{x})^{+} \rightarrow \widetilde{C}_{1}((\Sigma, \vec{z}), v, \vec{x})
$$

where we replace $\widetilde{C}_{0}((\Sigma, \vec{z}), v, \vec{x})$ by $\widetilde{C}_{0}((\Sigma, \vec{z}), v, \vec{x})^{+}$. (See (54).)

We first prove

$$
\operatorname{Index}\left(\widetilde{C}((\Sigma, \vec{z}), v, \vec{x})^{+}\right)^{\Gamma}=\operatorname{Index}\left(\widetilde{C}((\bar{\Sigma}, \vec{z}), \bar{v}, \overrightarrow{\bar{x}})^{+}\right) .
$$

Note the index of $\widetilde{C}((\Sigma, \vec{z}), v, \vec{x})^{+}$is the sum of indices of its components. Since the $\Gamma$-action is trivial on disc component, (57) is trivial for disc components. The part of sphere components of the left hand side is

$$
\sum_{b \in \bar{I}} \operatorname{Index}\left(D_{v_{a(b)}} \bar{\partial}\right)^{\Gamma_{a(b)}} .
$$

Here $\left\{S_{a(b)} \mid b \in \bar{I}\right\}$ is the complete set of representatives of the $\Gamma$-orbit space of the set of all sphere components of $(\Sigma, \vec{z}, v)$ and the map $v_{a(b)}$ is the restriction of $v$ to $S_{a(b)}$. The group $\Gamma_{a(b)}$ is a subgroup of $\Gamma$ consisting of the elements which preserve $S_{a(b)}$. We remark that $\Gamma_{a(b)}$ is a cyclic group. Hence we can apply Lemma 12.2 to show that (58) is equal to the sum of $\operatorname{Index}\left(D_{\bar{v}_{b}} \bar{\partial}\right)$. Here $\bar{v}_{b}$ is the restriction of $\bar{v}$ to $S_{a(b)} / \Gamma_{a(b)}=\bar{S}_{b} \subset \bar{\Sigma}$. (57) follows.

We next observe that there exists an exact sequence

$$
0 \rightarrow \widetilde{C}((\Sigma, \vec{z}), v, \vec{x}) \rightarrow \widetilde{C}((\Sigma, \vec{z}), v, \vec{x})^{+} \rightarrow \bigoplus_{x \in \operatorname{Sing} \Sigma} T_{v(x)} M \rightarrow 0 .
$$

Here the $\bigoplus_{x \in \operatorname{Sing} \Sigma} T_{v(x)} M$ is the sum over all singular points $x$ of $\Sigma$. 
We remark that $\Gamma$ action on $\bigoplus_{x \in \operatorname{Sing} \Sigma} T_{v(x)} M$ is by interchanging the factors. It follows that

$$
\operatorname{dim}\left(\bigoplus_{x \in \operatorname{Sing} \Sigma} T_{v(x)} M\right)^{\Gamma}=2 n \#((\operatorname{Sing} \Sigma) / \Gamma) .
$$

Note that $(\operatorname{Sing} \Sigma) / \Gamma \cong \operatorname{Sing} \bar{\Sigma}$. Therefore (57), (59) and a similar exact sequence for $\bar{\Sigma}$ imply the lemma.

We compare $\rho^{\Gamma_{a(b)}}\left(S_{a(b)}, \vec{p}_{a(b)}, o_{a(b)}\right)$ and $\bar{\rho}\left(\bar{S}_{b}, \overrightarrow{\bar{p}}_{b}, \bar{o}_{b}\right)$. Note that $\Gamma_{a(b)}$ is isomorphic to $\mathbb{Z}_{m_{a(b)}}$. If $m_{a(b)}=1, \Gamma_{a(b)}=\{e\}$ and so it is obvious that

$$
\rho^{\Gamma_{a(b)}}\left(S_{a(b)}, \vec{p}_{a(b)}, o_{a(b)}\right)=\bar{\rho}\left(\bar{S}_{b}, \vec{p}_{b}, \bar{o}_{b}\right) .
$$

Note that there is no free fixed point on $S_{a(b)}$.

From now on, we assume that $m_{a(b)}>1$. The marked point $o_{a(b)}$ is a $\Gamma_{a(b)}$ fixed point. Denote by $q_{a(b)}$ the other fixed point on $S_{a(b)}$. Let $\bar{q}_{b}$ be its image of the reduction in $\bar{S}_{b}$. There are two cases: $q_{a(b)} \in \vec{p}_{a(b)}$ or $q_{a(b)} \notin \vec{p}_{a(b)}$.

First consider the case $q_{a(b)} \in \vec{p}_{a(b)}, \bar{q}_{b} \in \vec{p}_{b}$. We claim that

$$
\rho^{\Gamma_{a(b)}}\left(S_{a(b)}, \vec{p}_{a(b)}, o_{a(b)}\right)=\bar{\rho}\left(\bar{S}_{b}, \overrightarrow{\bar{p}}_{b}, \bar{o}_{b}\right) .
$$

If $n_{a(b)}=1$, then $\bar{n}_{b}=1, \vec{p}_{a(b)}=q_{a(b)}, \vec{p}_{b}=\bar{q}_{b}$. We have

$$
\operatorname{Aut}\left(S_{a(b)}, q_{a(b)}, o_{a(b)}\right)^{\Gamma_{a(b)}} \cong \operatorname{Aut}\left(S_{a(b)}, q_{a(b)}, o_{a(b)}\right) .
$$

$\operatorname{Both} \operatorname{Aut}\left(S_{a(b)}, q_{a(b)}, o_{a(b)}\right)^{\Gamma_{a(b)}}$ and $\operatorname{Aut}\left(\bar{S}_{b}, \bar{q}_{b}, \bar{o}_{b}\right)$ are isomorphic to $\mathbb{C}^{*}$ and hence (61) follows.

In case $n_{a(b)} \geq 2$, we have $\bar{n}_{b} \geq 2$ and

$$
\operatorname{Conf}_{n_{a(b)}+1}^{\Gamma_{a(b)}}\left(S_{a(b)} ; \vec{p}_{a(b)}, o_{a(b)}\right) \cong \operatorname{Conf}_{\bar{n}_{b}+1}\left(\bar{S}_{b} ; \vec{p}_{b}, \bar{o}_{b}\right) .
$$

Hence, we obtain (61).

We next consider the case $q_{a(b)} \notin \vec{p}_{a(b)}, \bar{q}_{b} \notin \vec{p}_{b}$. In this case, the position of $\bar{q}_{b}$ in the reduced model is not a part of the data of the reduced model, $\left(\bar{S}_{b}, \vec{p}_{b}, \bar{o}_{b}\right)$. We claim

$$
\rho^{\Gamma_{a(b)}}\left(S_{a(b)}, \vec{p}_{a(b)}, o_{a(b)}\right)=\bar{\rho}\left(\bar{S}_{b}, \bar{o}_{b}\right)+2 .
$$

If $n_{a(b)}=0$, then $\bar{n}_{b}=0$. Note that $q_{a(b)}$ is a fixed point on $S_{a(b)}$. We find

$$
\operatorname{Aut}\left(S_{a(b)}, \vec{p}_{a(b)}, o_{a(b)}\right)^{\Gamma_{a(b)}} \cong \operatorname{Aut}\left(S_{a(b)}, q_{a(b)}, o_{a(b)}\right)
$$


which is isomorphic to $\mathbb{C}^{*}$. On the other hand, $\operatorname{Aut}\left(\bar{S}_{b}, \bar{o}_{b}\right)$ is isomorphic to the semi-direct product $\mathbb{C}^{*} \times \mathbb{C}$ that is the group of all affine maps $z \mapsto A z+B$ with $A \neq 0$. (62) follows.

Suppose that $\bar{n}_{b}=1$. Denote by $\bar{p}_{b}$ the unique point in $\vec{p}_{b}$. In this case, we find that $\operatorname{Conf}_{n_{a(b)}+1}^{\Gamma_{a(b)}}\left(\mathbb{C} P^{1}\right)$ is a point and $n_{a(b)} \geq 2$. On the other hand, $\operatorname{Aut}\left(\mathbb{C} P^{1}, \bar{p}_{b}, \bar{o}_{b}\right)$ is isomorphic to $\mathbb{C}^{*}$. (62) follows.

If $\bar{n}_{b}>1$, then $\vec{p}_{a(b)}$ consists of $\bar{n}_{b} \Gamma_{a(b)}$-orbits in $S_{a(b)} \backslash\left\{q_{a(b)}, o_{a(b)}\right\}$. Therefore we have

$$
\operatorname{dim}_{\mathbb{R}} \operatorname{Conf}_{n_{a(b)}+1}^{\Gamma_{a(b)}}\left(\mathbb{C} P^{1}\right)=2 \bar{n}_{b}-\operatorname{dim}_{\mathbb{R}} \mathbb{C}^{*}=2 \bar{n}_{b}-2,
$$

where $\mathbb{C}^{*} \cong \operatorname{Aut}\left(\mathbb{C} P^{1}, q_{a(b)}, o_{a(b)}\right)$. On the other hand,

$$
\operatorname{dim}_{\mathbb{R}} \operatorname{Conf}_{\bar{n}_{b}+1}\left(\mathbb{C} P^{1}\right)=2\left(\bar{n}_{b}+1\right)-\operatorname{dim}_{\mathbb{R}} \operatorname{Aut}\left(\mathbb{C} P^{1}\right)=2 \bar{n}_{b}-4 .
$$

We also obtain (62).

Note that $q_{a(b)}$ is a free fixed point if and only if $q_{a(b)} \notin \vec{p}_{a(b)}$. Therefore (62) is applied to the component $S_{a}$ if it contains a free fixed point. Otherwise (60),(61) apply.

We observe that the contribution of disc components to $\rho^{\Gamma}((\Sigma, \vec{z}), v)$ coincides with that of disc components to $\bar{\rho}((\bar{\Sigma}, \overrightarrow{\bar{z}}), \bar{v})$.

Combining these, we obtain

$$
\rho^{\Gamma}((\Sigma, \vec{z}), v)=\bar{\rho}((\bar{\Sigma}, \vec{z}), \bar{v})+\operatorname{dim}_{\mathbb{R}} C(F(\Gamma))^{\Gamma} .
$$

This formula together with Lemma 12.4 implies Proposition 12.3.

We next identify each side of Proposition 12.3 with the dimension of appropriate moduli space.

Definition 12.5. We say that $((\Sigma, \vec{z}), v, \vec{x})$ has the same combinatorial type as $\left(\left(\Sigma^{\prime}, \vec{z}^{\prime}\right), v^{\prime}, \vec{x}^{\prime}\right)$ if the following holds: There exists a homeomorphism $\Sigma \rightarrow \Sigma^{\prime}$ preserving all the marked points together with the order. We also assume that the restriction of $v$ to each component of $\Sigma$ is homologous to the restriction of $v^{\prime}$ to the corresponding component of $\Sigma^{\prime}$. We denote by $\mathbb{S}$ an equivalence class of this equivalence relation and call $\mathbb{S}$ the combinatorial type of $((\Sigma, \vec{z}), v, \vec{x})$.

We denote by

$$
\mathcal{M}_{k+1}^{\text {main }}\left(\beta ; P_{1}, \ldots, P_{k}\right)(\mathbb{S}, \Gamma)\left(=\mathcal{M}_{k+1}^{\text {main }}\left(\beta ; P_{1}, \ldots, P_{k}\right)(\mathbb{S}) \cong(\Gamma)\right)
$$


the moduli space of $((\Sigma, \vec{z}), v, \vec{x})$ with given combinatorial type $\mathbb{S}$ and its isotropy group $\Gamma$.

Let $\mathbb{S}$ be the combinatorial type of $((\Sigma, \vec{z}), v, \vec{x})$.

Lemma 12.5. The left hand side of Proposition 12.3 is

$$
\operatorname{dim}_{\mathbb{R}} \mathcal{M}_{k+1}^{\operatorname{main}}\left(\beta ; P_{1}, \ldots, P_{k}\right)(\mathbb{S}, \Gamma) .
$$

Proof. $\rho^{\Gamma}(\Sigma, \vec{z}, v)$ is the number of deformation parameters of $(\Sigma, \vec{z}, v)$ keeping the $\Gamma$-equivariance and the combinatorial type minus the dimension of the automorphism group of $(\Sigma, \vec{z})$. (Note that we did not include the parameter to resolve singularities of $\Sigma$. So this corresponds to the deformation keeping the combinatorial type.) Lemma 12.5 follows.

To study the right hand side of Proposition 12.3 we need some notation. For each $((\Sigma, \vec{z}), v, \vec{x})$ we consider its reduced model $((\bar{\Sigma}, \vec{z}), \bar{v}, \vec{x})$. We then add the image of elements in $F(\Gamma)$ to the reduced model as additional (interior) marked points. We denote them by $\vec{z}_{+}$and the resulting stable map by $\left(\left(\bar{\Sigma}, \vec{z}_{\vec{z}}, \vec{z}_{+}\right), \bar{v}, \vec{x}\right)$.

Note

$$
\# \vec{z}_{+}=\# F(\Gamma) / \Gamma \text {. }
$$

We call $\left(\left(\bar{\Sigma}, \vec{z}, \vec{z}_{+}\right), \bar{v}, \vec{x}\right)$ the marked reduced model of $((\Sigma, \vec{z}), v, \vec{x})$. In this way, we have obtained a natural assignment

$$
((\Sigma, \vec{z}), v, \vec{x}) \rightarrow\left(\left(\bar{\Sigma}, \overrightarrow{\bar{z}}, \vec{z}_{+}\right), \bar{v}, \vec{x}\right) .
$$

We note that the reduced model $((\bar{\Sigma}, \vec{z}), \bar{v}, \vec{x})$ may be unstable. On the other hand the marked reduced model is always stable.

Let us denote by $\overline{\mathbb{S}}$ the combinatorial type of the marked reduced model. We consider $\mathcal{M}_{k+1, \ell}^{\text {main }}\left(\bar{\beta} ; P_{1}, \ldots, P_{k}\right)(\overline{\mathbb{S}})$, the moduli space of marked reduced models with the combinatorial type $\overline{\mathbb{S}}$. Here $\ell$ is the order of $F(\Gamma) / \Gamma$. We now have:

Lemma 12.6. The right hand side of Proposition 12.3 is equal to

$$
\operatorname{dim}_{\mathbb{R}} \mathcal{M}_{k+1, \ell}^{\text {main }}\left(\bar{\beta} ; P_{1}, \ldots, P_{k}\right)(\overline{\mathbb{S}}) .
$$

Proof. In the same way as in the proof of Lemma 12.5, we find that

$$
\operatorname{dim}_{\mathbb{R}} \operatorname{Index}(\widetilde{C}((\bar{\Sigma}, \vec{z}), \bar{v}, \vec{x}))+\bar{\rho}((\bar{\Sigma}, \vec{z}), \bar{v})
$$


is the virtual dimension of the moduli space of reduced models. Adding marked points $\vec{z}_{+}$increases the dimension by $2 \# \vec{z}_{+}=2 \# F(\Gamma) / \Gamma$, which is equal to $\operatorname{dim}_{\mathbb{R}} C(F(\Gamma))^{\Gamma}$.

We next show the following.

Lemma 12.7. Suppose that $(M, J)$ is spherically positive and $\mathbb{S}$ contains at least one sphere component. Then we have

$$
\operatorname{dim}_{\mathbb{R}} \mathcal{M}_{k+1, \ell}^{\text {main }}\left(\bar{\beta} ; P_{1}, \ldots, P_{k}\right)(\overline{\mathbb{S}}) \leq \operatorname{dim}_{\mathbb{R}} \mathcal{M}_{k+1}^{\operatorname{main}}\left(\beta ; P_{1}, \ldots, P_{k}\right)-2
$$

Remark 12.6. We have not used spherical positivity of $J$ up to this point. Namely the proof of Lemma 12.7 is the only place where we use this assumption.

Proof. Let $J$ be a spherically positive almost complex structure. We consider a component $S_{a}$ of an element of $\mathcal{M}_{k+1}^{\text {main }}\left(\beta ; P_{1}, \ldots, P_{k}\right)(\mathbb{S})$. Let $\vec{w}_{a}, \vec{z}_{a}$ be the sets of all marked or singular points on $S_{a}, \partial S_{a}$, respectively. Let $v_{a}=\left.v\right|_{S_{a}}$. We put $k_{a}=\# \vec{w}_{a}+\# \vec{z}_{a} / 2$. We put $s_{a}=3$ if $S_{a}$ is a disc component and $s_{a}=6$ if $S_{a}$ is a sphere component. Set

$$
c(a)=2 c_{1}(M)\left[v_{a}\right]
$$

for a sphere component and

$$
c(a)=\mu_{L}\left(v_{a}\right)
$$

for a disc component.

We claim that

$$
c(a)+2 k_{a}-s_{a} \geq-2
$$

holds for the sphere components.

In fact, if the map is trivial on this component, then $2 k_{a} \geq 6$ and $c(a)=0$. (63) holds. If the map is nontrivial on this component, then we have $c(a) \geq 2$ by the choice of $J$ made in the beginning of the proof using spherical positivity. Since $2 k_{a} \geq 2$ and $s_{a}=6$ for a sphere component, (63) also holds. 
We put $\epsilon(a)=2$ for a sphere component and $\epsilon(a)=1$ for a disc component. It is easy to see from the index theorem that

$$
\begin{aligned}
& \operatorname{dim}_{\mathbb{R}} \mathcal{M}_{k+1}^{\operatorname{main}}\left(\beta ; P_{1}, \ldots, P_{k}\right)(\mathbb{S}) \\
& =\sum_{a \in I}\left(\epsilon(a) n+c(a)+2 k_{a}-s_{a}\right) \\
& \quad-(2 n) \# \operatorname{Sing}_{S^{2}} \mathbb{S}-n \# \operatorname{Sing}_{D^{2}} \mathbb{S}-\sum \operatorname{deg} P_{i},
\end{aligned}
$$

where $I$ is the set of all components of $\mathbb{S}, \# \operatorname{Sing}_{S^{2}} \mathbb{S}$ is the number of singular points which intersect with sphere components and $\# \operatorname{Sing}_{D^{2}} \mathbb{S}$ is the number of singular points which do not intersect with sphere components. We recall $2 n=\operatorname{dim} M$.

We have the similar identity for $\operatorname{dim}_{\mathbb{R}} \mathcal{M}_{k+1, \ell}^{\text {main }}\left(\bar{\beta} ; P_{1}, \ldots, P_{k}\right)(\overline{\mathbb{S}})$. Namely for each component $S_{b}$ of $\overline{\mathbb{S}}$ we define $\bar{k}_{b}, \bar{s}_{b}, \bar{c}(b), \bar{\epsilon}(b)$ and obtain

$$
\begin{aligned}
& \operatorname{dim}_{\mathbb{R}} \mathcal{M}_{k+1, \ell}^{\operatorname{main}}\left(\bar{\beta} ; P_{1}, \ldots, P_{k}\right)(\overline{\mathbb{S}}) \\
& =\sum_{b \in \bar{I}}\left(\bar{\epsilon}(b) n+\bar{c}(b)+2 \bar{k}_{b}-\bar{s}_{b}\right) \\
& \quad-(2 n) \# \operatorname{Sing}_{S^{2}} \overline{\mathbb{S}}-n \# \operatorname{Sing}_{D^{2}} \overline{\mathbb{S}}-\sum \operatorname{deg} P_{i}
\end{aligned}
$$

where $\bar{I}$ is the set of all components of $\overline{\mathbb{S}}$.

For each component $b \in \bar{I}$ we take $a(b) \in I$ such that $S_{a(b)}$ is a branched covering of $S_{b}$. (There may be several of them. We choose one of them.)

If $S_{b}$ is a disc component, we have

$$
\bar{c}(b)+2 \bar{k}_{b}-\bar{s}_{b}=c(a(b))+2 k_{a(b)}-s_{a(b)},
$$

since the automorphism group is trivial on the disc components.

We next prove the following:

\section{Sublemma 12.8.}

$$
\bar{c}(b)+2 \bar{k}_{b}-\bar{s}_{b} \leq c(a(b))+2 k_{a(b)}-s_{a(b)},
$$

if $S_{b}$ is a sphere component.

Proof. We have $\bar{s}_{b}=s_{a(b)}=6$. By the spherical positivity we have $\bar{c}(b) \geq 0$. It also follows that $\bar{c}(b) \leq c(a(b))$. If there is no free fixed point on $S_{a(b)}$, then $\bar{k}_{b} \leq k_{a(b)}$ and we are done. 
Now assume that there is a free fixed point on $S_{a(b)}$. In this case the degree, denoted by deg, of the map $S_{a(b)} \rightarrow S_{b}$ is greater than one.

We first consider the case when $\left[v_{b}\right] \neq 0$. Then by the definition of spherical positivity we have $c(a(b)) \geq 2$. Therefore

$$
\bar{c}(b)=\frac{c(a(b))}{\operatorname{deg}} \leq c(a(b))-2 .
$$

(Note that $c(a), \bar{c}(b)$ are even numbers for the sphere components.) On the other hand, since there exists at most one free fixed point on $S_{a(b)}$, it follows that

$$
\bar{k}_{b} \leq k_{a(b)}+1
$$

The sublemma follows in this case.

We next assume $\left[v_{b}\right]=0$. Then $k_{a(b)} \geq 3$ by stability. Namely there exist at least 3 singular or marked points. The component $S_{b}$ is identified with the quotient of $S_{a(b)}$ by the cyclic group $\Gamma_{a(b)}$ of order $\operatorname{deg} \in\{2,3, \ldots\}$. Let $d$ be the distance of $S_{a(b)}$ from the disc components. Let $o_{a(b)}$ be the singular point on $S_{a(b)}$ where $S_{a(b)}$ is attached with $\Sigma_{d-1}$. Clearly $o_{a(b)}$ is a fixed point of $\Gamma_{a(b)}$. Since there is a free fixed point on $S_{a(b)}$, no other singular points are fixed by $\Gamma_{a(b)}$. In other words the image in $S_{b}$ of the singular points of $S_{a(b)}$ consists of $1+\left(k_{a(b)}-1\right) / \operatorname{deg}$ points. Therefore

$$
\bar{k}_{b}=2+\frac{k_{a(b)}-1}{\operatorname{deg}} .
$$

Since $k_{a(b)} \geq 3$, we derive

$$
\bar{k}_{b} \leq k_{a(b)}
$$

The proof of Sublemma 12.8 is now complete.

It follows from Sublemma 12.8 and (66) that

$$
\sum_{b \in \bar{I}}\left(\bar{\epsilon}(b) n+\bar{c}(b)+2 \bar{k}_{b}-\bar{s}_{b}\right) \leq \sum_{b \in \bar{I}}\left(\epsilon(a(b)) n+c(a(b))+2 k_{a(b)}-s_{a(b)}\right) .
$$

(63) implies that for each sphere component $S_{a}(a \in I)$ we have

$$
2 n+c(a)+2 k_{a}-s_{a} \geq 2 n-2 .
$$


Note that the number of sphere components of $\mathbb{S}$ and of $\overline{\mathbb{S}}$ are equal to $\# \operatorname{Sing}_{S^{2}} \mathbb{S}$ and to $\# \operatorname{Sing}_{S^{2}} \overline{\mathbb{S}}$, respectively. Therefore we have

$$
\begin{aligned}
& \sum_{a \in I}\left(\epsilon(a) n+c(a)+2 k_{a}-s_{a}\right)-(2 n-2) \# \operatorname{Sing}_{S^{2}} \mathbb{S} \\
& \geq \sum_{b \in \bar{I}}\left(\epsilon(a(b)) n+c(a(b))+2 k_{a(b)}-s_{a(b)}\right)-(2 n-2) \# \operatorname{Sing}_{S^{2}} \overline{\mathbb{S}} .
\end{aligned}
$$

We remark that $\operatorname{Sing}_{D^{2}} \overline{\mathbb{S}}=\operatorname{Sing}_{D^{2}} \mathbb{S}$. The Formulas (64), (65), (67) and (68) imply

$$
\begin{aligned}
& \operatorname{dim}_{\mathbb{R}} \mathcal{M}_{k+1}^{\text {main }}\left(\bar{\beta} ; P_{1}, \ldots, P_{k}\right)(\overline{\mathbb{S}}) \\
& \leq \operatorname{dim}_{\mathbb{R}} \mathcal{M}_{k+1}^{\text {main }}\left(\beta ; P_{1}, \ldots, P_{k}\right)(\mathbb{S})+2\left(\# \operatorname{Sing}_{S^{2}} \mathbb{S}-\# \operatorname{Sing}_{S^{2}} \overline{\mathbb{S}}\right) .
\end{aligned}
$$

On the other hand, we have

$$
\begin{aligned}
& \operatorname{dim}_{\mathbb{R}} \mathcal{M}_{k+1}^{\operatorname{main}}\left(\beta ; P_{1}, \ldots, P_{k}\right) \\
& =\operatorname{dim}_{\mathbb{R}} \mathcal{M}_{k+1}^{\operatorname{main}}\left(\beta ; P_{1}, \ldots, P_{k}\right)(\mathbb{S})+2 \# \operatorname{Sing}_{S^{2}} \mathbb{S}+\# \operatorname{Sing}_{D^{2}} \mathbb{S} .
\end{aligned}
$$

Since $\# \operatorname{Sing}_{S^{2}} \overline{\mathbb{S}} \geq 1$, we obtain the lemma.

Now we are in the position to complete the proof of Proposition 12.1. Let $\Gamma \neq\{1\}$ be an abstract group. By Proposition 12.3 and Lemmas 12.5, 12.6, 12.7 we have

$$
\begin{aligned}
\operatorname{dim}_{\mathbb{R}} \mathcal{M}_{k+1}^{\text {main }}\left(\beta ; P_{1}, \ldots, P_{k}\right)(\mathbb{S}, \Gamma) & =\operatorname{dim}_{\mathbb{R}} \mathcal{M}_{k+1}^{\text {main }}\left(\bar{\beta} ; P_{1}, \ldots, P_{k}\right)(\overline{\mathbb{S}}) \\
& \leq \operatorname{dim}_{\mathbb{R}} \mathcal{M}_{k+1}^{\text {main }}\left(\beta ; P_{1}, \ldots, P_{k}\right)-2
\end{aligned}
$$

By definition (12), we have

$$
\max _{i} d\left(\mathcal{M}_{k+1}^{\operatorname{main}}\left(\beta ; P_{1}, \ldots, P_{k}\right) ; \Gamma ; i\right)=\max _{\mathbb{S}} \operatorname{dim}_{\mathbb{R}} \mathcal{M}_{k+1}^{\operatorname{main}}\left(\beta ; P_{1}, \ldots, P_{k}\right)(\mathbb{S}, \Gamma) .
$$

Here the maximum in the right hand side is taken over $\mathbb{S}$ such that $\mathcal{M}_{k+1}^{\text {main }}\left(\beta ; P_{1}, \ldots, P_{k}\right)(\mathbb{S}, \Gamma)$ is nonempty. Now Proposition 12.1 follows from (69).

Remark 12.7. Consider the case when $\mathbb{S}$ is a union of a disc $D^{2}$ and a sphere $S^{2}$ where $c_{1}(M)\left[\left.v\right|_{S^{2} *}\left(\left[S^{2}\right]\right)\right]=0$. We assume that $\left.v\right|_{S^{2}}$ is a cyclic multiple cover of a map $u$. The reduced model consists of the same configuration where the map $\left.v\right|_{S^{2}}$ is replaced by $u$. The virtual dimension of the reduced model is the same as the virtual dimension of $\mathcal{M}_{k+1}^{\operatorname{main}}\left(\beta ; P_{1}, \ldots, P_{k}\right)(\mathbb{S})$, the moduli space with combinatorial type $\mathbb{S}$. Since there is one sphere bubble, the virtual dimension of the moduli 
space $\mathcal{M}_{k+1}^{\text {main }}\left(\beta ; P_{1}, \ldots, P_{k}\right)(\mathbb{S})$ is the virtual dimension of $\mathcal{M}_{k+1}^{\text {main }}\left(\beta ; P_{1}, \ldots, P_{k}\right)$ minus 2 .

However, since there is one free fixed point on the sphere bubble, it follows that the virtual dimension of marked reduced model $\mathcal{M}_{k+1,1}^{\text {main }}\left(\bar{\beta} ; P_{1}, \ldots, P_{k}\right)(\overline{\mathbb{S}})$ is equal to the virtual dimension of $\mathcal{M}_{k+1}^{\text {main }}\left(\beta ; P_{1}, \ldots, P_{k}\right)$. Namely (53) does not hold.

This is the reason why we assume spherical positivity in this paper.

If we can use the reduced model in place of the marked reduced model, this condition would be removed. However it seems rather difficult to show the compatibility of the normally conical perturbation with the process of forgetting extra marked points in the marked reduced model.

\section{Filtered $A_{\infty}$ Algebra over $\mathbb{Z}_{2}$}

13.1. Construction of a filtered $A_{n, K}$ structure over $\mathbb{Z}_{2}$. We now use Theorem 3.1 and Proposition 12.1 to prove Theorem 1.1 (except Item (4), that is the case of $\Lambda_{0, \text { nov }}^{\mathbb{Z}}$ coefficient). Actually we need the following relative version of Theorem 3.1. We say a global section $\mathfrak{s}^{\prime}=\left\{s_{p}^{\prime}\right\}$ of a space with Kuranishi structure to be normally conical if each of $s_{p}^{\prime}$ is normally conical in the sense of Definition 10.1. Let $X$ be a space with Kuranishi structure with corners. The boundary of $\partial X$ is decomposed into spaces $\partial_{c} X$ with Kuranishi structures such that $\partial_{c} X$ intersects with $\partial_{c^{\prime}} X$ along the corner $\left(=\partial_{c c^{\prime}} X\right)$ of $X$ with codimension $\geq 2$. (More precisely, there exists a map from $\partial_{c} X$ to the boundary of $X$ so that it is injective outside the codimension 2 corner $\partial_{c c} X$. See [7] right before Definition A1.30.)

Let $X$ has a Kuranishi sturucture with corners. We denote by $\stackrel{\circ}{S}_{k} X$ the set of the points of $X$ that lies on the codimension $k$ corner. If $p \in \stackrel{\circ}{S}_{k} X$, then we may take its Kuranishi neighborhood $V_{p} / \Gamma_{p}$ so that

$$
V_{p}=V_{p}^{\prime} \times[0, \rho)^{k} .
$$

(Here $V_{p}^{\prime}$ is an open set of the Euclidean space.) The finite group $\Gamma_{p}$ acts on $V_{p}$ and preserves its stratification as a manifold with corners. So the action of $\Gamma_{p}$ exchanges the second factor $[0, \rho)^{k}$. We write

$$
\partial_{c} V_{p}=V_{p}^{\prime} \times[0, \rho)^{c-1} \times\{0\} \times[0, \rho)^{k-c}
$$


Assumption 13.1. All the elements of $\Gamma_{p}$ preserve each of $\partial_{c} V_{p}$.

We remark that $\mathcal{M}_{k+1}^{\operatorname{main}}(\beta)$ satisfies Assumption 13.1. In fact in the case of $X=\mathcal{M}_{k+1}^{\text {main }}(\beta)$ the coordinates $[0,1)^{m}$ that parametrize the direction normal to the corner, is the glueing parameter of the boundary singular points. On the other hand, the element of the isotropy group $\Gamma_{p}$ is the automorphism that is supported on sphere bubbles. Therefore the $\Gamma_{p}$ action on $[0,1)^{m}$ is trivial.

Since $\mathcal{M}_{k+1}^{\text {main }}\left(\beta ; P_{1}, \ldots, P_{k}\right)$ is a fiber product of $\mathcal{M}_{k+1}^{\text {main }}(\beta)$ with $P_{i}$, Assumption 13.1 is also satisfied for $\mathcal{M}_{k+1}^{\operatorname{main}}\left(\beta ; P_{1}, \ldots, P_{k}\right)$.

Under Assumption 13.1 we can easily prove the following:

Lemma 13.1. The Kuranishi neighborhood and obstruction bundle of a neighborhood of codimension $k$ corner $\stackrel{\circ}{S}_{k} X$ is the product of the induced Kuranshi structure on $\stackrel{\circ}{S}_{k} X$ and $[0, \rho)^{k}$.

Namely we can take the diffeomorphism

$$
V_{p} \cong V_{p}^{\prime} \times[0, \rho)^{k}
$$

so that it is $\Gamma_{p}$ equivariant and is compatible with the coordinate change, for each $p \in \stackrel{\circ}{S}_{k} X$. Moreover we have an equivariant isomorphism of the obstruction bundles

$$
\left.E_{p} \cong E_{p}\right|_{V_{p}^{\prime}} \times[0, \rho)^{k}
$$

We remark that the Kuranishi map $s_{p}$ may not be constant in the $[0, \rho)^{k}$ direction (under the identifications $(70),(71)$.)

Theorem 13.2. Let $X$ be a space with Kuranishi structure with corners and tangent bundle. We assume that $X$ satisfies Assumption 13.1. Suppose that we have global sections $\mathfrak{s}_{c}^{\prime}$ of $\partial_{c} X$ for each $c$ such that they coincide with each other at the intersections $\partial_{c c^{\prime}} X$. We assume $\mathfrak{s}_{c}^{\prime}$ are normally conical and satisfy the conclusion of Theorem 3.1. Then we can extend them to a normally conical global section of $X$ satisfying the conclusion of Theorem 3.1.

Remark 13.2. Actually we can prove Theorem 13.2 without assuming Assumption 13.1. We assume it only to simplify the proof. In our application this assumption is satisfied. 
Proof. We put

$$
S_{k} X=\bigcup_{m \geq k} \stackrel{\circ}{S}_{m} X .
$$

Let $U_{\epsilon}\left(S_{k} X\right)$ be the $\epsilon$ neighborhood of $S_{k} X$. (Here we take a metric on $X$ and fix it.) Let $N$ be the smallest positive integer such that $S_{N+1} X$ is empty. We will prove the following by induction on $m_{0}=0,1, \ldots, N$.

Lemma 13.3. There exists $\epsilon_{k, m}$ for $m \leq m_{0}$ and $k=N-m, \ldots, N$ and $a$ normally conical global section $\mathfrak{s}_{m}$ on a Kuranishi neighborhood of

$$
\bigcup_{k=N-m}^{N} U_{\epsilon_{k, m}}\left(S_{k} X\right)
$$

such that they have the following properties.

(1) $\mathfrak{s}_{m}$ satisfies the conclusion of Theorem 3.1.

(2) The restriction of $\mathfrak{s}_{m}$ to the intersection of (72) and $\partial_{c} X$ coincides with the restriction of $\mathfrak{s}_{c}^{\prime}$.

(3) $\epsilon_{k, m+1}<\epsilon_{k, m}$ if $k \geq N-m$. Moreover $\mathfrak{s}_{m+1}=\mathfrak{s}_{m}$ on a Kuranishi neighborhood of $\bigcup_{k=N-m}^{N} U_{\epsilon_{k, m+1}}\left(S_{k} X\right)$.

Proof. We first prove the first step $m_{0}=0$ of the induction. We need to find $\epsilon_{N, 0}$ and $\mathfrak{s}_{0}$ on $U_{\epsilon_{N, 0}}\left(S_{N} X\right)$. By assumption $S_{N} X$ has a Kuranishi structure without boundary. The restriction of various $\mathfrak{s}_{c}^{\prime}$ to $S_{N} X$ gives a global section on $S_{N} X$ that satsifies the conclusion of Theorem 3.1. We will extend it to a neighborhood of $S_{N} X$.

We take an isomorphism

$$
F: S_{N} X \times[0,1)^{N} \rightarrow X
$$

to an open neighborhood. (More precisely (73) is a family of isomorphisms (70),(71) that are $\Gamma_{p}$ equivariant and compatible with the coordinate change.

We remark that

$$
\left([0,1)^{N}, \bigcup_{c=1}^{N}\left([0,1)^{c-1} \times\{0\} \times[0,1)^{N-c}\right)\right)
$$

is $\mathrm{PL}$ isomorphic to

$$
\left([0,1) \times B^{N-1},\{0\} \times B^{N-1}\right)
$$


as a pair. Here $B^{N-1}=\left\{x \in \mathbb{R}^{N-1}|| x \mid<1\right\}$ is an open ball of dimension $N-1$.

We remark that we are given a family of global sections $\mathfrak{s}_{c}^{\prime}$ on a Kuranishi neighborhood of

$$
S_{N} X \times \bigcup_{c=1}^{N}\left([0,1)^{c-1} \times\{0\} \times[0,1)^{N-c}\right)
$$

so that they are compatible on the overlapped part. So we use the isomorphism between (74) and (75) to extend the section $\mathfrak{s}_{c}^{\prime}$ to a Kuranishi neighborhood of

$$
S_{N} X \times[0,1) \times B^{N-1} \cong S_{N} X \times[0,1)^{N}
$$

so that it coicides with $\mathfrak{s}_{c}^{\prime}$ on (a Kuranishi neighborhood of)

$$
S_{N} X \times\{0\} \times B^{N-1} \cong S_{N} X \times \bigcup_{c=1}^{N}\left([0, \rho)^{c-1} \times\{0\} \times[0, \rho)^{N-c}\right) .
$$

Namely we take the extension so that it is constant in the $[0,1)$ factor. Then it is easy to see that extended section has the required properties. We have thus proved the case $m_{0}=0$ of Lemma 13.3.

We next discuss the induction step for $m_{0}<N$. We remark that $\stackrel{\circ}{S}_{N-m_{0}} X$ has a Kuranishi structure without boundary. We take $\epsilon_{k, m_{0}}<\epsilon_{k, m_{0}-1}$ for $k>N-m_{0}$. We put

$$
U\left(m_{0}-1\right)=\bigcup_{k=N-m_{0}+1}^{N} U_{\epsilon_{k, m_{0}-1}}\left(S_{k} X\right)
$$

and

$$
U^{-}\left(m_{0}-1\right)=\bigcup_{k=N-m_{0}+1}^{N} U_{\epsilon_{k, m_{0}}}\left(S_{k} X\right)
$$

and

$$
S_{N-m_{0}}^{-} X=S_{N-m_{0}} X \backslash U^{-}\left(m_{0}-1\right) .
$$

We have a piecewise smooth isomorphism between a Kuranishi neighborhood in $X$ of $S_{N-m_{0}}^{-} X$ and the product of a Kuranishi neighborhood (in $\stackrel{\circ}{S}_{N-m_{0}} X$ ) of $S_{N-m_{0}}^{-} X$ and $[0,1)^{m_{0}}$ in the sense above.

We also have a piecewise smooth isomorphism between Kuranishi neighborhoods of

$$
S_{N-m_{0}}^{-} X \times[0,1)^{m_{0}}
$$


and of

$$
S_{N-m_{0}}^{-} X \times[0,1) \times B^{m_{0}-1}
$$

so that $S_{N-m_{0}}^{-} X \times\{0\} \times B^{m_{0}-1}$ becomes the intersection with $\bigcup_{c} \partial_{c} X$ under the identifications.

By induction hypothesis we have $\mathfrak{s}_{m_{0}-1}$. We take its restriction to (a Kuranishi neighborhood of)

$$
U\left(m_{0}-1\right) \cap\left(S_{N-m_{0}}^{-} X \times[0,1)^{m_{0}}\right) .
$$

We may identify a Kuranishi neighborhood of $U\left(m_{0}-1\right) \cap\left(S_{N-m_{0}}^{-} X \times[0,1)^{m_{0}}\right)$ with a Kuranishi neighborhood of $\left(U\left(m_{0}-1\right) \cap S_{N-m_{0}}^{-} X\right) \times[0,1)^{m_{0}}$. By construction we also have a piecewise smooth diffeomorphism

$$
\begin{aligned}
& \left(U\left(m_{0}-1\right) \cap S_{N-m_{0}}^{-} X\right) \times[0,1)^{m_{0}} \\
& \cong\left(U\left(m_{0}-1\right) \cap S_{N-m_{0}}^{-} X\right) \times[0,1) \times B^{m_{0}-1}
\end{aligned}
$$

such that $\mathfrak{s}_{m_{0}-1}$ is constant in the $[0,1)$ factor by this identification.

By changing the identification between (76) and (77) if necessary we may assume that the identification between (76) and (77) coincides with (78) except in a small neighborhood of $\partial U\left(m_{0}-1\right) \cap S_{N-m_{0}}^{-} X$.

Thus we define $\mathfrak{s}_{m_{0}}$ so that it is constant in the $[0,1)$ factor of $(77)$ under the above identifications. Then it is easy to see that extended section has the required properties. We remark that we use $m_{0}<N$ here. In fact in case $m_{0}=N$, the stratum $S_{0}^{-} X$ is not contained in any of $\partial_{c} X$ so we can not define $\mathfrak{s}_{m_{0}}$ by taking it independent of $[0,1)$ factor.

We have thus proved the lemma for $m_{0}<N$. So we have defined a global section on a (Kuranishi) neighborhood of the boundary $\partial X$. We can then extend it by the following relative version Propositin 13.4 of Theorem 3.1. It proves the case of $m_{0}=N$. The proof of Lemma 13.3 is complete.

By taking $m_{0}=N$ Lemma 13.3 implies Theorem 13.2.

Proposition 13.4. Let $X$ be a space with Kuranishi structure that has a tangent bundle, $K \subset X$ a compact subset and $U$ a Kuranishi neighborhood of $K$.

We take a good coordinate system of $X$. 
Suppose we have a sequence of normally conical strongly piecewise smooth global sections $\mathfrak{s}_{\epsilon}$ on $U$ such that it satisfies Theorem 3.1 (i)(ii)(iii) and converges to the Kuranishi map in $C^{0}$ sense as $\epsilon \rightarrow 0$.

Then there exists a sequence of normally conical strongly piecewise smooth global sections $\mathfrak{s}_{\epsilon}^{\prime}$ on $X$ such that it satisfies Theorem 3.1 (i)(ii)(iii), coincides with $\mathfrak{s}_{\epsilon}$ on a Kuranishi neighborhood of $K$ and converges to the Kuranishi map in $C^{0}$ sense as $\epsilon \rightarrow 0$.

Moreover if $f: X \rightarrow Y$ is a strongly smooth map from $X$ to a manifold $Y$, and if we have a triangulation of an intersection of $\left(\mathfrak{s}_{\epsilon}\right)^{-1}(0)$ with a Kuranishi neighborhood of $K$, such that $f$ induces a piecewise smooth map $\left(\mathfrak{s}_{\epsilon}\right)^{-1}(0) \rightarrow Y$, then we can extend the triangulation to $\left(\mathfrak{s}_{\epsilon}^{\prime}\right)^{-1}(0)$ so that $f$ induces a piecewise smooth $\operatorname{map}\left(\mathfrak{s}_{\epsilon}^{\prime}\right)^{-1}(0) \rightarrow Y$.

We remark that Theorem 3.1 is proved by induction using Proposition 10.1, which is a relative version of Proposition 7.1. (See Section 10.) So we can prove Proposition 13.4 in the same way as Theorem 3.1.

We can use Theorem 13.2 in place of Theorem A1.23 [7] (which is a similar result for multisection) together with Proposition 12.1 to prove Theorems 1.1, 1.2 in the same way as in Chapter 7 [7]. Below we explain this construction, which is parallel to Section 7.2 [7].

Now we consider $\mathcal{M}_{k+1}^{\operatorname{main}}\left(\beta ; P_{1}, \ldots, P_{k}\right)$. By Proposition 7.1.2 [7], its boundary is decomposed into the following:

$$
\mathcal{M}_{k+1}^{\text {main }}\left(\beta ; P_{1}, \ldots, \partial_{i} P_{j}, \ldots, P_{k}\right)
$$

where $\partial_{i} P_{j}$ is the $i$-th factor of the singular simplex $P_{j}$ and

$$
\left(\mathcal{M}_{k_{1}+1}^{\text {main }}\left(\beta_{1}\right)_{e v_{0}} \times_{e v_{i}} \mathcal{M}_{k_{2}+1}^{\text {main }}\left(\beta_{2}\right)\right) e v^{\prime} \times_{L^{k}}\left(P_{1} \times \cdots \times P_{k}\right)
$$

where $k_{1}+k_{2}=k+1$ and $i \in\left\{1, \ldots, k_{2}\right\}$. The evaluation map

$$
e v^{\prime}=\left(e v_{1}^{\prime}, \ldots, e v_{k}^{\prime}\right): \mathcal{M}_{k_{1}+1}^{\text {main }}\left(\beta_{1}\right)_{e v_{0}} \times_{e v_{i}} \mathcal{M}_{k_{2}+1}^{\text {main }}\left(\beta_{2}\right) \rightarrow L^{k},
$$

which we use to take the fiber product in $(79 \mathrm{~b})$, is defined by

$$
e v_{j}^{\prime}(\mathfrak{x}, \mathfrak{y})= \begin{cases}e v_{j}(\mathfrak{x}) & \text { if } j<i, \\ e v_{j-i+1}(\mathfrak{y}) & \text { if } i \leq j<i+k_{2}, \\ e v_{j-k_{2}+1}(\mathfrak{x}) & \text { if } j \geq i+k_{2} .\end{cases}
$$


Therefore $(79 b)$ is identified with

$$
\begin{aligned}
\mathcal{M}_{k_{1}+1}^{\text {main }}\left(\beta_{1}\right) \times_{L^{k_{1}}}( & P_{1} \times \cdots \times P_{i-1} \\
& \left.\times \mathcal{M}_{k_{2}+1}^{\operatorname{main}}\left(\beta_{2} ; P_{i}, \ldots, P_{i+k_{2}-1}\right) \times P_{i+k_{2}} \times \cdots \times P_{k}\right) .
\end{aligned}
$$

Proposition 13.5. Let $E$ and $K$ be fixed integers. Then there is a system of global sections $\mathfrak{s}_{\beta, P_{1}, \ldots, P_{k}}^{\prime}$ of $\mathcal{M}_{k+1}^{\operatorname{main}}\left(\beta ; P_{1}, \ldots, P_{k}\right)$ for each $k \leq K$ and $\beta$ with $\omega(\beta) \leq E$ with the following properties:

(i) They are normally conical and satisfy the conclusion of Theorem 3.1.

(ii) They are compatible at the boundary component (79a).

(iii) The zero set of each section $\mathfrak{s}_{\beta, P_{1}, \ldots, P_{k}}^{\prime}$ is given with triangulation. Each simplex of it is identified with a smooth singular chain of $L$ by the (strongly smooth extension of) the map ev .

(iv) Let $P^{\prime}$ be a simplex of the zero set of $\mathfrak{s}_{\beta, P_{i}, \ldots, P_{i+k_{2}-1}}$. Then the restriction of $\mathfrak{s}_{\beta, P_{1}, \ldots, P_{k}}^{\prime}$ at the subset

$$
\mathcal{M}_{k_{1}+1}^{\operatorname{main}}\left(\beta_{1}\right) \times_{L^{k_{1}}}\left(P_{1} \times \cdots \times P_{i-1} \times P^{\prime} \times P_{i+k_{2}} \times \cdots \times P_{k}\right)
$$

of (80) coincides with $\mathfrak{s}_{\beta_{1}, P_{1}, \ldots, P_{i-1}, P^{\prime}, P_{i+k_{2}}, \ldots, P_{k}}$.

Remark 13.3. The way how the statement (iv) above is made is slightly imprecise. Namely we need to describe the relation between obstruction bundles to make a precise statement. See Proposition 7.2.35 [7].

Proof. The proof of Proposition 13.5 is by induction. We consider the order on the set of pairs $(\beta, k)$ such that $(\beta, k)<\left(\beta^{\prime}, k^{\prime}\right)$ if either $(1) \omega[\beta]<\omega\left[\beta^{\prime}\right]$ or $(2)$ $\omega[\beta]=\omega\left[\beta^{\prime}\right], k<k^{\prime}$.

The conditions (ii) and (iv) describe the global section $\mathfrak{s}_{k, \beta, P_{1}, \ldots, P_{k}}^{\prime}$ at the boundary. We can check that they are consistent at the corners of codimension $\geq 2$. (See Lemma 7.2.55 [7].) Then by Theorem 3.1 we can extend it to the whole moduli space $\mathcal{M}_{k+1}^{\operatorname{main}}\left(\beta ; P_{1}, \ldots, P_{k}\right)$. We refer Section $7.2[7]$ for the detail.

Now we fix a system of global sections $\mathfrak{s}_{\beta, P_{1}, \ldots, P_{k}}^{\prime}$ as in Proposition 13.5 for each $k, \beta, P_{1}, \ldots, P_{k}$ with $k \leq K$ and $\omega(\beta) \leq E$. Let $\mathcal{M}_{k+1}^{\operatorname{main}}\left(\beta ; P_{1}, \ldots, P_{k}\right)^{\mathfrak{s}^{\prime}}$ be the zero set of $\mathfrak{s}_{\beta, P_{1}, \ldots, P_{k}}^{\prime}$. We fixed its triangulation in (iii).

Up to this point, spherical positivity is not assumed. Now we assume that $(M, \omega, J)$ is spherically positive. Then Proposition 12.1 implies the following. 
Lemma 13.6. If $P^{\prime}$ is a simplex of $\mathcal{M}_{k+1}^{\operatorname{main}}\left(\beta ; P_{1}, \ldots, P_{k}\right)^{\mathfrak{s}^{\prime}}$ with

$$
\operatorname{dim} P^{\prime} \geq \operatorname{dim} \mathcal{M}_{k+1}^{\operatorname{main}}\left(\beta ; P_{1}, \ldots, P_{k}\right)-2
$$

then the interior of $P^{\prime}$ does not intersect with $\mathcal{M}_{k+1}^{\operatorname{main}}\left(\beta ; P_{1}, \ldots, P_{k}\right)(\Gamma)$ for $\Gamma \neq$ $\{1\}$.

Let $P_{a}^{\prime}(a \in A)$ be the set of all simplices of dimension $\mathcal{M}_{k+1}^{\text {main }}\left(\beta ; P_{1}, \ldots, P_{k}\right)$. We put

$$
e v_{0 *}\left[\mathcal{M}_{k+1}^{\text {main }}\left(\beta ; P_{1}, \ldots, P_{k}\right)^{\mathfrak{s}^{\prime}}\right]=\sum_{a} P_{a}^{\prime}
$$

this is a smooth singular chain of $L$ with $\mathbb{Z}_{2}$ coefficients. We now put

$$
\mathfrak{m}_{k, \beta}\left(P_{1}, \ldots, P_{k}\right)=e v_{0 *}\left[\mathcal{M}_{k+1}^{\operatorname{main}}\left(\beta ; P_{1}, \ldots, P_{k}\right)^{\mathfrak{s}^{\prime}}\right] .
$$

We use Lemma 13.6 and Proposition 13.5 (i), (iii) to obtain the following formula:

$$
\begin{aligned}
& \partial \mathfrak{m}_{k, \beta}\left(P_{1}, \ldots, P_{k}\right)+\sum_{i} \mathfrak{m}_{k, \beta}\left(P_{1}, \ldots, \partial P_{i}, \ldots P_{k}\right) \\
& =\sum_{\beta_{1}+\beta_{2}=\beta} \sum_{k_{1}+k_{2}=k+1} \sum_{i=1, \ldots, k_{2}} \mathfrak{m}_{k_{1}, \beta_{1}}\left(P_{1}, \ldots, P_{i-1},\right. \\
& \left.\mathfrak{m}_{k_{2}, \beta_{2}}\left(P_{i}, \ldots, P_{i+k_{2}-1}\right), P_{i+k_{2}}, \ldots, P_{k}\right),
\end{aligned}
$$

where we put $\mathfrak{m}_{1, \beta_{0}}=\partial\left(\beta_{0}=0\right.$ is zero in $\left.H_{2}(M ; L)\right)$. Taking the sum over $\beta$, (83) gives rise to the $A_{\infty}$ formula which defines an $A_{\infty}$ algebra. More precisely, the formula gives rise to a filtered $A_{n, K}$ structure in the sense defined in Section 7.2 [7]. Here the integer $K$ is as above and $n$ is determined by $E$ as follows. We consider the subset of $\mathbb{R}$ defined by

$$
\left\{\left(\omega[\beta], \mu_{L}(\beta)\right) \mid \mathcal{M}_{1}(\beta) \neq \emptyset\right\} \subset \mathbb{R} \times \mathbb{Z} .
$$

We take the additive submonoid of $\mathbb{R} \times \mathbb{Z}$ generated by this set and denote it by $\mathfrak{G}(L, J)$. For an element $\beta \in \mathfrak{G}(L, J)$ we put

$$
\|\beta\|=\sup \left\{n \mid \exists \beta_{i} \in \mathfrak{G}(L, J), \sum_{i=1}^{n} \beta_{i}=\beta\right\} .
$$

We say $(n, K)<\left(n^{\prime}, K^{\prime}\right)$ if either (1) $n+K<n^{\prime}+K^{\prime}$ or (2) $n+K=n^{\prime}+K^{\prime}$, $n<n^{\prime}$. Filtered $A_{n, K}$ structure is by definition a system of operators $\mathfrak{m}_{k, \beta}$ which are for $k \leq K$ and $\beta$ with $(\|\beta\|, k) \leq(n, K)$ such that it satisfies (83). (See Definition $7.2 .70[7]$.) 
Remark 13.4. See Subsection 7.2.3 [7] for the reason why we need to restrict to $k \leq K$ and $\omega(\beta) \leq E$ for the proof of Proposition 13.5.

13.2. Construction of a filtered $A_{n, K}$ homomorphism over $\mathbb{Z}_{2}$. We can work in the same way as Section 7.2 [7] to go from an $A_{n, K}$ structure (for arbitrary but fixed $n, K)$ to an $A_{\infty}$ structure. For this purpose we should also use the construction of filtered $A_{n, K}$ homomorphisms which we describe later in this subsection. See Lemma 13.9. In this way we associate a filtered $A_{\infty}$ structure to the singular chain complex of a Lagrangian submanifold over $\mathbb{Z}_{2}$ coefficients in the spherically positive case. Then it was proved in Theorem 5.4.2 [6] that this $A_{\infty}$ structure on the singular chain complex induces a canonical filtered $A_{\infty}$ structure on the cohomology group, which we call the canonical model. (We use Lemma 14.1 for this purpose.) To show that the canonical model depends only on the connected component of $J \in \mathcal{J}_{(M, \omega)}^{c_{1}>0}$ up to an isomorphism we proceed as follows. Let $J_{0}, J_{1}$ be elements of $\mathcal{J}_{(M, \omega)}^{c_{1}>0}$. We use each one of them to construct our filtered $A_{\infty}$ structure on singular chain complex. (We also fix a system of global sections, triangulation of their zero sets etc..) Let $\left\{J_{\rho}\right\}_{\rho \in[0,1]}$ be a path in $\mathcal{J}_{(M, \omega)}^{c_{1}>0}$ joining them. We now consider the moduli space $\mathcal{M}_{k+1}^{\operatorname{main}}\left(\left\{J_{\rho}\right\}_{\rho}: \beta ; \operatorname{top}(\rho) ; P_{1}, \ldots, P_{k}\right)$ which was introduced by Definition 4.6.10 [6].

We here recall its definition. Let $(\Sigma, \vec{z})$ be a bordered Riemann surface of genus 0 with $k$ of boundary marked points. We consider each of disc component of $\Sigma$ and attach tree of sphere components rooted on it. We thus decompose

$$
\Sigma=\bigcup_{a=0}^{m} \Sigma_{a}
$$

where each of $\Sigma_{a}$ contains exactly one disc components. Let $\Sigma_{0}$ be the component which contains 0 -th marked point $z_{0}$. We define a partial order $<$ on the set of components as follows: $\Sigma_{a}<\Sigma_{b}$ if every path joining $\Sigma_{a}$ to $z_{0}$ intersect $\Sigma_{b}$. We consider $\rho_{a} \in[0,1]$ for each $a=0, \ldots, m$ such that if $\Sigma_{a}<\Sigma_{b}$ then $\rho_{a} \leq \rho_{b}$. We call $\left\{\rho_{a}\right\}_{a=0}^{m}$ the time allocation. We consider the set of $\left((\Sigma, \vec{z}),\left\{\rho_{a}\right\}, v\right)$ such that

(1) $(\Sigma, \vec{z})$ is a bordered Riemann surface of genus 0 with $k$ of boundary marked points.

(2) $\left\{\rho_{a}\right\}_{a=0}^{m}$ is its time allocation.

(3) $v:(\Sigma, \partial \Sigma) \rightarrow(M, L)$ is a continuous map.

(4) On each component $\Sigma_{a}$ the restriction of $v$ to $\Sigma_{a}$ is $J_{\rho_{a}}$-holomorphic. 
(5) We assume the stability in the following sense: the set of biholomorphic map $\varphi: \Sigma \rightarrow \Sigma$ such that $\varphi\left(z_{i}\right)=z_{i}, v \circ \varphi=v$ is finite.

We say $((\Sigma, \vec{z}),\{\rho\}, v) \sim\left(\left(\Sigma^{\prime}, \vec{z}^{\prime}\right),\left\{\rho_{a^{\prime}}^{\prime}\right\}, v^{\prime}\right)$ if there exists a biholomorphic map $\varphi: \Sigma^{\prime} \rightarrow \Sigma$ such that $\varphi\left(z_{i}^{\prime}\right)=z_{i}, v \circ \varphi=v^{\prime}$ and $\rho_{a}=\rho_{a^{\prime}}$, where $\varphi\left(\Sigma_{a^{\prime}}\right)=\Sigma_{a}$. We denote by $\mathcal{M}_{k+1}^{\operatorname{main}}\left(\left\{J_{\rho}\right\}_{\rho}: \beta\right.$; $\left.\operatorname{top}(\rho)\right)$ the set of $\sim$ equivalence classes of the triple $\left((\Sigma, \vec{z}),\left\{\rho_{a}\right\}, v\right)$ satisfying (1)-(5). Using the evaluation map

$$
e v=\left(e v_{0}, \ldots, e v_{k}\right): \mathcal{M}_{k+1}^{\text {main }}\left(\left\{J_{\rho}\right\}_{\rho}: \beta ; \operatorname{top}(\rho)\right) \rightarrow L^{k+1},
$$

we obtain $\mathcal{M}_{k+1}^{\text {main }}\left(\left\{J_{\rho}\right\}_{\rho}: \beta ; \operatorname{top}(\rho) ; P_{1}, \ldots, P_{k}\right)$ for smooth singular chains $P_{1}, \ldots, P_{k}$. It is a space with Kuranishi structure with tangent space. Its boundary is decomposed into three parts which correspond to the following 5 possibilities

(A) The moduli space

$$
\mathcal{M}_{k+1}^{\text {main }}\left(\left\{J_{\rho}\right\}_{\rho}: \beta ; \operatorname{top}(\rho) ; P_{1}, \ldots, \partial_{i} P_{j}, \ldots, P_{k}\right)
$$

where $\partial_{i} P_{j}$ is the $i$-th face of $P_{j}$.

(B) One of the time allocation becomes 0 .

(C) One of the time allocation becomes 1 .

(D) Two time allocation $\rho_{a}$ and $\rho_{b}$ coincides, and corresponding component $\Sigma_{a}$ and $\Sigma_{b}$ intersects.

(E) One disc component splits into two in the limit.

One can prove (D) and (E) above cancel to each other. (See Figures 4.6.2 and 4.6.3 [6].) Therefore the actual boundary is only (A),(B),(C) above.

We next describe (B) and (C) by fiber product. (See Subsection 7.2.9 [7] for details.)

(B) is described as the union of the following fiber product over $k_{1}, k_{2}, \beta_{1}, \beta_{2}, i$ with $k_{1}+k_{2}=k-1, \beta_{1}+\beta_{2}=\beta, i=1, \ldots, k_{2}$ :

$$
\begin{aligned}
& \mathcal{M}_{k_{1}+1}^{\text {main }}\left(\left\{J_{\rho}\right\}_{\rho}: \beta_{1} ; \operatorname{top}(\rho)\right) \\
& \times_{L^{k_{1}}}\left(P_{1} \times \cdots P_{i-1} \times \mathcal{M}_{k_{2}+1}^{\text {main }}\left(J_{0}, \beta_{2} ; P_{i}, \ldots, P_{i+k_{2}-1}\right) \times P_{i+k_{2}} \times \cdots \times P_{k}\right) .
\end{aligned}
$$

Here we write $\mathcal{M}_{k_{2}+1}^{\operatorname{main}}\left(J_{0}, \beta_{2} ; P_{i}, \ldots, P_{i+k_{2}-1}\right) \quad$ in place of $\mathcal{M}_{k_{2}+1}^{\text {main }}\left(\beta_{2} ; P_{i}, \ldots, P_{i+k_{2}-1}\right)$ to clarify the almost complex structure we use for definition. 
(C) is described as follows. We consider $1 \leq \ell_{1} \leq \cdots \leq \ell_{m} \leq \ell_{m+1}=k$. Then (C) is a union of the following fiber products:

$$
\mathcal{M}_{m+1}^{\text {main }}\left(J_{1}, \beta^{\prime}\right) \times_{L^{m}} \prod_{i=1}^{m} \mathcal{M}_{\ell_{i+1}-\ell_{i}+1}^{\text {main }}\left(\left\{J_{\rho}\right\}_{\rho}: \beta_{i} ; \operatorname{top}(\rho) ; P_{\ell_{i}}, \ldots, P_{\ell_{i+1}-1}\right) .
$$

Here we take union over $m,\left\{\ell_{1}, \ldots, \ell_{m+1}\right\}, \beta^{\prime}, \beta_{i}$ such that $\ell_{i}$ is as above and $\beta^{\prime}+\sum \beta_{i}=\beta$. Note we include the case $\ell_{i+1}=\ell_{i}+1$ and $\beta_{i}=0 \in H_{2}(M, L ; \mathbb{Z})$. In that case we put

$$
\mathcal{M}_{1}^{\text {main }}\left(\left\{J_{\rho}\right\}_{\rho}: 0 ; \operatorname{top}(\rho) ; P_{\ell_{i}}\right)=P_{\ell_{i}}
$$

by definition.

In a way similar to Proposition 13.5 we can prove the following. Let $E$ and $K$ be fixed integers. We take and fix a system of global sections $\mathfrak{s}_{J_{0}, \beta, P_{1}, \ldots, P_{k}}^{\prime}$ of $\mathcal{M}_{k+1}^{\text {main }}\left(J_{0}, \beta ; P_{1}, \ldots, P_{k}\right)$ and $\mathfrak{s}_{J_{1}, \beta, P_{1}, \ldots, P_{k}}^{\prime}$ of $\mathcal{M}_{k+1}^{\text {main }}\left(J_{1}, \beta ; P_{1}, \ldots, P_{k}\right)$ as in Proposition 13.5.

Proposition 13.7. There is a system of global sections $\mathfrak{s}_{\left\{J_{\rho}\right\}, \beta, P_{1}, \ldots, P_{k}}^{\prime}$ of

$$
\mathcal{M}_{k+1}^{\text {main }}\left(\left\{J_{\rho}\right\}_{\rho}: \beta ; \operatorname{top}(\rho) ; P_{1}, \ldots, P_{k}\right)
$$

for each $k \leq K$ and $\beta$ with $\omega(\beta) \leq E$ with the following properties.

(i) They are normally conical and satisfy the conclusion of Theorem 3.1.

(ii) They are compatible at the boundary component (84).

(iii) Each simplex $P^{\prime}$ of the zero set of $\mathfrak{s}_{J_{0}, \beta_{1}, P_{i+1}, \ldots, P_{i+k_{2}-1}}$ is identified with a smooth singular chain of $L$ by the (strongly smooth extention of) the map $e v_{0}$. Then the restriction of $\mathfrak{s}_{\left\{J_{\rho}\right\}, \beta, P_{1}, \ldots, P_{k}}^{\prime}$ to

$$
\mathcal{M}_{k_{1}+1}^{\text {main }}\left(\left\{J_{\rho}\right\}_{\rho}: \beta_{1} ; \operatorname{top}(\rho) ; P_{1}, \ldots P_{i-1}, P^{\prime}, P_{i+k_{2}-1}, \ldots, P_{k}\right)
$$

coincides with $\mathfrak{s}_{\left\{J_{\rho}\right\}, \beta_{1}, P_{1}, \ldots . P_{i-1}, P^{\prime}, P_{i+k_{2}-1}, \ldots, P_{k}}$. Here (88) is a part of (85).

(iv) Each of the zero set of the global section $\mathfrak{s}_{\left\{J_{\rho}\right\}_{\rho}, \beta_{i}, P_{\ell_{i}}, \ldots, P_{\ell_{i+1}-1}}$ of

$$
\mathcal{M}_{\ell_{m+1}-\ell_{m}+1}^{\text {main }}\left(\left\{J_{\rho}\right\}_{\rho}: \beta_{i} ; \operatorname{top}(\rho) ; P_{\ell_{i}}, \ldots, P_{\ell_{i+1}-1}\right)
$$

is given with triangulation. Let $P_{i}^{\prime}$ be a simplex of the zero set of $\mathfrak{s}_{\left\{J_{\rho}\right\}_{\rho}, \beta_{i}, P_{\ell_{i}}, \ldots, P_{\ell_{i+1}-1}}^{\prime}$. Then the restriction of $\mathfrak{s}_{\left\{J_{\rho}\right\}_{\rho}, \beta, P_{1}, \ldots, P_{k}}^{\prime}$ to the corresponding subset of (86) coincides with $\mathfrak{s}_{J_{0}, \beta_{0}, P_{1}^{\prime}, \ldots, P_{m}^{\prime}}$. 
Note the same remark as Remark 13.3 applies to (iii) and (iv) above.

The proof of Proposition 13.7 is similar to the proof of Proposition 13.5. In other words it is similar to the proof of Proposition 7.2.100 [7]. We use global single valued sections (and Theorem 13.2) here in place of multisections used in [7].

Lemma 13.8. We assume $J_{\rho} \in \mathcal{J}_{(M, \omega)}^{c_{1}>0}$ for each $\rho$. Let $\Gamma$ be a nontrivial finite group. Then we have

$$
\begin{aligned}
& \operatorname{dim} \mathcal{M}_{k+1}^{\operatorname{main}}\left(\left\{J_{\rho}\right\}_{\rho}: \beta ; \operatorname{top}(\rho) ; P_{1}, \ldots, P_{k}\right) \\
& -d\left(\mathcal{M}_{k+1}^{\operatorname{main}}\left(\left\{J_{\rho}\right\}_{\rho}: \beta ; \operatorname{top}(\rho) ; P_{1}, \ldots, P_{k}\right) ; \Gamma ; i\right) \geq 2 .
\end{aligned}
$$

See (29) for the notation of the second term. The proof is the same as the proof of Proposition 12.1.

It implies that for a global section of $\mathcal{M}_{k+1}^{\text {main }}\left(\left\{J_{\rho}\right\}_{\rho}: \beta\right.$; top $\left.(\rho) ; P_{1}, \ldots, P_{k}\right)$ satisfying the conclusion of Theorem 13.2, the simplex which lies in the fixed point locus of some non-trivial group $\Gamma$ is codimension $\geq 2$. Thus we can define its virtual fundamental chain over $\mathbb{Z}_{2}$. We use it to define

$$
\mathfrak{f}_{k, \beta}\left(P_{1}, \ldots, P_{k}\right)=e v_{0 *}\left[\mathcal{M}_{k+1}^{\operatorname{main}}\left(\left\{J_{\rho}\right\}_{\rho}: \beta ; \operatorname{top}(\rho) ; P_{1}, \ldots, P_{k}\right)^{\mathfrak{s}^{\prime}}\right]
$$

in the same way as (81). We put also

$$
\mathfrak{f}_{1, \beta_{0}}=\text { identity }
$$

for $\beta_{0}=0 \in H_{2}(M, L ; \mathbb{Z})$. (Compare (87).) Then we have the following formula: (In the next formula we write $\mathfrak{m}_{k, \beta}^{J_{0}}, \mathfrak{m}_{k, \beta}^{J_{1}}$ to distinguish two filtered $A_{n, K}$ structures which depend almost complex structures $J_{0}, J_{1}$ together with other choices made.)

$$
\begin{aligned}
& \sum \mathfrak{m}_{\beta^{\prime}, m}^{J_{1}}\left(\mathfrak{f}_{\beta_{1}, \ell_{2}-\ell_{1}+1}\left(P_{1}, \ldots, P_{\ell_{1}}\right), \ldots, \mathfrak{f}_{\beta_{m}, \ell_{m+1}-\ell_{m}+1}\left(P_{m}, \ldots, P_{k}\right)\right) \\
& =\sum \mathfrak{f}_{k_{1}, \beta_{1}}\left(P_{1}, \ldots, P_{i-1}, \mathfrak{m}_{k_{2}, \beta_{2}}^{J_{0}}\left(P_{i}, \ldots, P_{i+k_{2}-1}\right), P_{i+k_{2}}, \ldots, P_{k}\right) .
\end{aligned}
$$

Here the sum in the right hand side is taken over $k_{1}, k_{2}, \beta_{1}, \beta_{2}, i$ with $k_{1}+k_{2}=$ $k-1, \beta_{1}+\beta_{2}=\beta, i=1, \ldots, k_{2}$ and the sum in the left hand side is taken over all $m,\left\{\ell_{1}, \ldots, \ell_{m+1}\right\}, \beta^{\prime}, \beta_{i}$ such that $1 \leq \ell_{1} \leq \cdots \leq \ell_{m} \leq \ell_{m+1}=k$ and $\beta^{\prime}+\sum \beta_{i}=\beta$.

In fact, the left hand side except those for $\beta^{\prime}=0, m=1$ corresponds (C) (in other words (iv) Proposition 13.7). The right hand side except those for $\beta_{2}=0$, 
$k_{2}=0$ corresponds (B) (in other words (iii) Proposition 13.7). The right hand side for $\beta_{2}=0, k_{2}=0$ corresponds (A) (in other words (i) Proposition 13.7). Compatibility spelled out in Proposition 13.7 and Lemma 13.8 implies that the boundary of $\mathfrak{f}_{\beta, k}\left(P_{1}, \ldots, P_{k}\right)$, that is nothing but the left hand side for $\beta^{\prime}=0$, $m=1$, is equal to the sum of the terms corresponding to $(\mathrm{A}),(\mathrm{B}),(\mathrm{C})$. It implies (91).

(91) implies that

$$
\mathfrak{f}_{k}=\sum_{\beta} T^{\omega[\beta]} e^{\mu_{L}(\beta) / 2} \mathfrak{f}_{k, \beta}
$$

define a filtered $A_{n, K}$ homomorphism. By definition $\mathfrak{f}_{1} \equiv$ identity $\bmod \Lambda_{0, \text { nov }}^{+}$, where $\Lambda_{0, \text { nov }}^{+}$is the set of sums (2) such that all $\lambda_{i}$ are strictly positive. Therefore Theorem 4.2.45 [6] implies that $\mathfrak{f}_{k}$ defines a homotopy equivalence of filtered $A_{n, K}$ structures.

13.3. From $A_{n, K}$ structure to $A_{\infty}$ structure. Now we go back to the construction of filtered $A_{\infty}$ structure. We recall $(n, K)<\left(n^{\prime}, K^{\prime}\right)$ if either (1) $n+K<n^{\prime}+K^{\prime}$ or $(2) n+K=n^{\prime}+K^{\prime}, n<n^{\prime}$.

Lemma 13.9. If $\left(C, \mathfrak{m}_{k, \beta}\right)$ (resp. $\left.\left(C^{\prime}, \mathfrak{m}_{k, \beta}^{\prime}\right)\right)$ is a filtered $A_{n, K}$ (resp. $A_{n^{\prime}, K^{\prime}}$ ) algebra with $(n, K)<\left(n^{\prime}, K^{\prime}\right)$. Suppose there exists a filtered $A_{n, K}$ homomorphism $\mathfrak{f}: C \rightarrow C^{\prime}$ which is a homotopy equivalence in the sense of filtered $A_{n, K}$ homomorphism. Then we can extend the filtered $A_{n, K}$ structure of $C$ to a filtered $A_{n^{\prime}, K^{\prime}}$ structure without changing operations which already exist in the filtered $A_{n, K}$ structure. We then can also extend $\mathfrak{f}$ to a homotopy equivalence in the sense of filtered $A_{n^{\prime}, K^{\prime}}$ homomorphism.

This is Theorem 7.2.72 [7]. We already explained the construction of filtered $A_{n, K}$ structure on the smooth singular chain complex of $L$ for arbitrary but fixed $n, K$. We also proved that they are homotopy equivalent to each other. So we can use Lemma 13.9 to construct a filtered $A_{\infty}$ structure. (See Subsection 7.2.9 [7] for detail of this construction.)

Then as we mentioned before we obtain a filtered $A_{\infty}$ structure on the cohomology group $H\left(L ; \Lambda_{0}^{\mathbb{Z}_{2}}\right)$. We remark that this is ordinary cohomology group and is not a Floer cohomology. Namely we take the boundary operator with respect to the usual boundary operator which does not include $\mathfrak{m}_{1, \beta}$ for $\beta \neq 0$. 
By construction the filtered $A_{\infty}$ algebra obtained on $H\left(L ; \Lambda_{0}^{\mathbb{Z}_{2}}\right)$ is independent, up to the homotopy equivalence, of the choices we have made of filtered $A_{n, K}$ algebra for any $n, K$. Then we can use the following.

Lemma 13.10. Let $(C,\{\mathfrak{m}\})$ and $\left(C^{\prime},\left\{\mathfrak{m}^{\prime}\right\}\right)$ be filtered $A_{\infty}$ algebras over $\Lambda_{0, \text { nov }}^{R}$ for a finite field $R$. We assume that they are homotopy equivalent as filtered $A_{n, K}$ algebras for any $n, K$. We assume that $C$ and $C^{\prime}$ are finitely generated free $\Lambda_{0, \text { nov }}^{R}$ modules. Then they are homotopy equivalent as filtered $A_{\infty}$ algebras.

Proof. Let $V(n, K)$ be the set of all filtered $A_{n, K}$ homotopy equivalence from $(C,\{\mathfrak{m}\})$ to $\left(C^{\prime},\left\{\mathfrak{m}^{\prime}\right\}\right)$. Using the fact that $R$ is a finite field we can easily see that this is a finite set. By assumption it is nonempty. Therefore the projective limit

$$
\underset{\lim }{\longleftarrow}(n, K)
$$

with respect to the order $<$ on $(n, K)$ is nonempty. It is easy to see that this implies that $(C,\{\mathfrak{m}\})$ is homotopy equivalent to $\left(C^{\prime},\left\{\mathfrak{m}^{\prime}\right\}\right)$ as filtered $A_{\infty}$ algebra.

We remark that Lemma 13.10 is related to Lemma 7.2.177 [7]. In other words, we are taking the short cut given in Subsection 7.2.11 [7] using the fact that our ground field $R$ is $\mathbb{Z}_{2}$.

We can prove that our filtered $A_{\infty}$ algebra has a unit in the same way as in Section $7.3[7]$.

Remark 13.5. In Section 7.2 [7], we introduced the notion of generation to the smooth singular chains and organized the induction in the way depending to the generation of $P_{i}$. We did so in order to work on the countably generated subcomplex of smooth singular chains. (Actually by slightly modifying the argument there we can work on finitely generated chain complex.)

Actually we can use the whole smooth singular chains and do not need to introduce generations. (Since our global section $\mathfrak{s}_{\beta, P_{1}, \ldots, P_{k}}^{\prime}$ may depend on $P_{i}$ 's, we can apply Baire's category theorem, which we need to apply general position argument, even in case we have uncountably many singular chains.) That is the way taken in this section.

This may slightly simplify the argument of Section 7.2 [7]. On the other hand, some people (including some of the authors of the present paper) may feel happier 
to use only countably many (or finitely many) singular chains, since to make a choice of perturbations, uncountably many times (for each of the singular chains $\left.P_{1}, \ldots, P_{k}\right)$ is rather a wild use of the axiom of choice.

The proof of Theorem 1.2 is similar and parallel to [6], [7] and so we omit it.

\section{The CASE OF $\mathbb{Z}$ COEFFicients}

So far we study the case of $\mathbb{Z}_{2}$ coefficients. The case of $\mathbb{Z}$ coefficients goes in mostly a similar way. In this section we discuss it. We focus on the points which are new in $\mathbb{Z}$ coefficients and avoid repeating the same material.

We first discuss the construction of a filtered $A_{\infty}$ structure on the singular chain complex $S\left(L ; \Lambda_{0, \text { nov }}^{\mathbb{Z}}\right)$ of a relatively spin Lagrangian submanifold $L$ of a spherically positive symplectic manifold $(M, \omega)$. The construction is mostly the same as in Section 13. Using the relatively spin structure we can define an orientation of the moduli space and hence the simplices in Propositions 13.5 and 13.7 are oriented. Moreover, the orientation is compatible with the description of the boundary. Thus we can define the operator $\mathfrak{m}_{k, \beta}$ by using the virtual fundamental chain (81) regarded as a singular chain with $\mathbb{Z}$ coefficients. We need sign that is the same as in [7] Definition 8.4.1. The formula (83) becomes the following

$$
\begin{aligned}
& \mathfrak{m}_{1,0} \mathfrak{m}_{k, \beta}\left(P_{1}, \ldots, P_{k}\right)+\sum_{i}(-1)^{*} \mathfrak{m}_{k, \beta}\left(P_{1}, \ldots, \mathfrak{m}_{1,0}\left(P_{i}\right), \ldots, P_{k}\right) \\
& =-\sum_{\substack{\beta_{1}+\beta_{2}=\beta, k_{1}+k_{2}=k+1 ; \\
\beta_{1} \neq 0 \text { or } k_{1} \neq 1,1 \\
\beta_{2} \neq 0 \text { or } k_{2} \neq 1}} \sum_{i}(-1)^{*} \mathfrak{m}_{k_{1}, \beta_{1}}\left(P_{1}, \ldots, \mathfrak{m}_{k_{2}, \beta_{2}}\left(P_{i}, \ldots, P_{i+k_{2}-1}\right), \ldots, P_{k}\right),
\end{aligned}
$$

where $*=\sum_{j=1}^{i-1} \operatorname{deg}^{\prime} P_{j},\left(\operatorname{deg}^{\prime} P_{j}=\operatorname{deg} P_{j}+1\right)$ and $\mathfrak{m}_{1,0}=(-1)^{n} \partial,(n=\operatorname{dim} L)$. See (3.5.15) and Definition 3.5.6 of [6]. The sign is checked in the same way as in [7] Section 8.5. Furthermore in the same way, (91) holds with an appropriate sign. (See [7] Subsection 8.9.1.)

We thus obtain the following. 
(1) We have a pair of sequences $(n(I), K(I)) \rightarrow(\infty, \infty)$ as $I \rightarrow \infty$ and a filtered $A_{n(I), K(I)}$ structure $\left\{\mathfrak{m}_{k, \beta}^{I, J}\right\}$ on $S\left(L ; \Lambda_{0, \text { nov }}^{\mathbb{Z}}\right)$ for each $I$. (Here we take the whole smooth singular chain complex and do not introduce generation. See Remark 13.5 $5^{1}$ )

(2) For $I<I^{\prime}$, we can prove that $\left(S\left(L ; \Lambda_{0, \text { nov }}^{\mathbb{Z}}\right),\left\{\mathfrak{m}_{k, \beta}^{I, J}\right\}\right)$ is homotopy equivalent to $\left(S\left(L ; \Lambda_{0, \text { nov }}^{\mathbb{Z}}\right),\left\{\mathfrak{m}_{k, \beta}^{I^{\prime}, J}\right\}\right)$ as filtered $A_{n(I), K(I)}$ structure.

(3) We use an appropriate obstruction theory and Item (2) to enhance the filtered $A_{n(I), K(I)}$ structure $\left(S\left(L ; \Lambda_{0, \text { nov }}^{\mathbb{Z}}\right),\left\{\mathfrak{m}_{k, \beta}^{I, J}\right\}\right)$ to a filtered $A_{\infty}$ structure.

(4) For each $I$ the filtered $A_{\infty}$ structure obtained in Item (3) is independent of the choice of almost complex structure $J$ (in the given connected component of $\left.\mathcal{J}_{(M, \omega)}^{c_{1}>0}\right)$ and of other choices involved up to filtered $A_{n(I), K(I)}$ homotopy equivalence.

Now there are some difference between the case of $\mathbb{Z}$ coefficients and the case of Section 13. Namely Lemma 13.10 does not hold over $\mathbb{Z}$. See [7] Remark 7.2.181. Instead we proceed as follows.

(5) Let $\left(S\left(L ; \Lambda_{0, \text { nov }}^{\mathbb{Z}}\right),\left\{\mathfrak{m}_{k, \beta}^{I, J}\right\}\right)$ and $\left(S\left(L ; \Lambda_{0, \text { nov }}^{\mathbb{Z}}\right),\left\{\mathfrak{m}_{k, \beta}^{\prime, I, J^{\prime}}\right\}\right)$ be filtered $A_{n(I), K(I)}$ structures obtained by different choices of $J$ etc. Then there exists a pair of sequences $\left(n^{\prime}(I), K^{\prime}(I)\right) \rightarrow(\infty, \infty)$ such that $\left(S\left(L ; \Lambda_{0, \text { nov }}^{\mathbb{Z}}\right),\left\{\mathfrak{m}_{k, \beta}^{I, J}\right\}\right)$ is homotopy equivalent to $\left(S\left(L ; \Lambda_{0, \text { nov }}^{\mathbb{Z}}\right),\left\{\mathfrak{m}_{k, \beta}^{\prime, I, J^{\prime}}\right\}\right)$ as filtered $A_{n^{\prime}(I), K^{\prime}(I)}$ algebras.

(6) Moreover for $I<I^{\prime}$ the following diagram commutes as a diagram of filtered $A_{n^{\prime}(I), K^{\prime}(I)}$ homomorphisms.

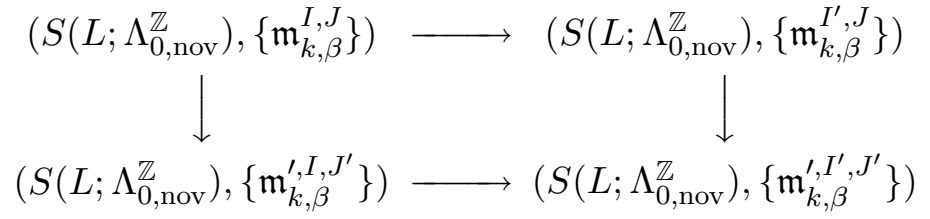

Here horizontal arrows are ones of Item (2) and the vertical arrows are ones of Item (5).

We can prove Items (5) and (6) in a way similar to [7] Subsection 7.2.10 by modifying in the following manner: We replace multisections used in [7] Subsection 7.2 .10 by a single valued normally conical section by using our assumption (spherically positivity) and Theorem 13.2.

\footnotetext{
${ }^{1}$ We may use generation and discuss in the same way as in [7] Section 7 as well.
} 
(7) Then we can use [7] Lemma 7.2.129 inductively to show that the filtered $A_{\infty}$ structure in Item (3) is independent of $J$ (in the given connected component of $\left.\mathcal{J}_{(M, \omega)}^{c_{1}>0}\right)$ and other choices up to filtered $A_{\infty}$ homotopy equivalence.

Remark 14.1. We did not claim that homotopy equivalence obtained in Item (7) above is independent of the choices up to filtered $A_{\infty}$ homotopy. This independence was proved in [7] Subsections 7.2.12-7.2.13 over $\mathbb{Q}$. We can actually prove it in the same way over $\mathbb{Z}$. Namely we define the notion of homotopy of homotopies as in [7] Subsection 7.2.12 and proceed in the same way as in [7] Subsections 7.2.12-7.2.13. The rather cumbersome homological algebra in [7] Subsections 7.2.12-7.2.13 is carefully designed so that it works over arbitrary coefficients.

In order to prove the independence of homotopy equivalence up to filtered $A_{\infty}$ homotopy over a finite field, we can again use the short cut used in [7] Subsection 7.2.11.

We next mention the reason why we assumed that $H(L ; \mathbb{Z})$ is torsion free in Item (4) of Theorem 1.1. In the last section we first construct a filtered $A_{\infty}$ structure on the singular chain complex of $L$ and reduce it to the singular cohomology. For this purpose we use the following lemma.

Let $\left(C,\left\{\mathfrak{m}_{k}\right\}_{k=0}^{\infty}\right)$ be a filtered $A_{\infty}$ algebra over $\Lambda_{0, \text { nov }}^{R}$. We put

$$
C=\bar{C} \otimes_{R} \Lambda_{0, \text { nov }}^{R},
$$

where $\bar{C}$ is a graded free $R$ module. The $R$ reduction $\overline{\mathfrak{m}}_{1}: \bar{C} \rightarrow \bar{C}$ of $\mathfrak{m}_{1}$ satisfies $\overline{\mathfrak{m}}_{1} \circ \overline{\mathfrak{m}}_{1}=0$ and hence $\left(\bar{C}, \overline{\mathfrak{m}}_{1}\right)$ is a chain complex.

Lemma 14.1. Suppose we have a direct sum decomposition $\bar{C}=\bar{D} \oplus \bar{D}^{\prime}$ of $R$ module such that $\left(\bar{D}, \overline{\mathfrak{m}}_{1}\right)$ is a subcomplex of $\left(\bar{C}, \overline{\mathfrak{m}}_{1}\right)$ and the inclusion $\left(\bar{D}, \overline{\mathfrak{m}}_{1}\right) \rightarrow$ $\left(\bar{C}, \overline{\mathfrak{m}}_{1}\right)$ is a chain homotopy equivalence. Then a filtered $A_{\infty}$ structure is induced on $D=\bar{D} \otimes_{R} \Lambda_{0, \text { nov }}^{R}$ such that it is homotopy equivalent to $C$.

The proof of Lemma 14.1 is the same as the proof of [6] Theorem 5.4.2 and so is omitted.

Example 14.2. (1) When $R$ is a field, we always have a splitting

$$
\bar{C}=H\left(\bar{C}, \overline{\mathfrak{m}}_{1}\right) \oplus \bar{C}^{\prime}
$$


such that $H\left(\bar{C}, \overline{\mathfrak{m}}_{1}\right)$ (with zero boundary operator) is chain homotopy equivalent to $\left(\bar{C}, \overline{\mathfrak{m}}_{1}\right)$. Therefore by Lemma 14.1 we obtain a filtered $A_{\infty}$ structure on $H\left(\bar{C}, \overline{\mathfrak{m}}_{1}\right) \otimes_{R} \Lambda_{0, \text { nov }}^{R}$. We used this fact in the last section.

(2) In the case $R=\mathbb{Z}$ (or any Dedekind domain), the splitting (94) exists if $H\left(\bar{C}, \overline{\mathfrak{m}}_{1}\right)$ is torsion free. (Note that any finitely generated torsion free module over a Dedekind domain is projective.) Therefore under the assumption that $H(L ; R)$ is torsion free, the filtered $A_{\infty}$ structure on the singular chain complex induces one on its singular cohomology.

Remark 14.3. In general, we can obtain a filtered $A_{\infty}$ structure on a finite dimensional complex over $\Lambda_{0 \text {,nov }}^{\mathbb{Z}}$ using some additional information on $L$ as follows:

(1) Let us fix a Morse function $f$ on $L$. We obtain a Morse complex $C(L ; f)$. It is a chain complex over $\mathbb{Z}$ and is torsion free as $\mathbb{Z}$ module. Using Lemma 14.1 and a filtered $A_{\infty}$ structure on the singular chain complex we obtain a filtered $A_{\infty}$ structure on $C(L ; f) \otimes \Lambda_{0, \text { nov }}^{\mathbb{Z}}$. See [9] Theorem 5.1.

(2) Let us take either a simplicial decomposition or a CW decomposition of $L$. Then we have a finite dimensional chain complex $C(L ; \mathbb{Z})$ which is free over $\mathbb{Z}$ and is chain homotopy equivalent to the singular chain complex of $L$. So we obtain a filtered $A_{\infty}$ structure on $C\left(L ; \Lambda_{0, \text { nov }}^{\mathbb{Z}}\right)$.

To prove the last claim in Item (4) of Theorem 1.1 it suffices to prove the following.

Lemma 14.2. Let $\left(S\left(L ; \Lambda_{0, \text { nov }}^{\mathbb{Z}}\right),\left\{\mathfrak{m}_{k}^{\mathbb{Z}}\right\}\right)$ be the filtered $A_{\infty}$ algebra in Theorem 1.1 and $\left(S\left(L ; \Lambda_{0, \text { nov }}^{\mathbb{Q}}\right),\left\{\mathfrak{m}_{k}^{\mathbb{Q}}\right\}\right)$ the filtered $A_{\infty}$ algebra in $[6]$ Theorem 3.5.11. Then $\left(S\left(L ; \Lambda_{0, \text { nov }}^{\mathbb{Z}}\right),\left\{\mathfrak{m}_{k}^{\mathbb{Z}}\right\}\right) \otimes \mathbb{Q}$ is homotopy equivalent to $\left(S\left(L ; \Lambda_{0, \text { nov }}^{\mathbb{Q}}\right),\left\{\mathfrak{m}_{k}^{\mathbb{Q}}\right\}\right)$.

Proof. We consider the moduli space

$$
\mathcal{M}_{k+1}^{\operatorname{main}}\left(\{J\}_{\rho}: \beta ; \operatorname{top}(\rho) ; P_{1}, \ldots, P_{k}\right)
$$

defined in Section 13. Here we fix $J \in \mathcal{J}_{(M, \omega)}^{c_{1}>0}$ and use the constant family $\rho \mapsto J$ of almost complex structures. We use appropriate perturbation of this moduli space to define the required homotopy equivalence.

Note that we took a system of single valued normally conical sections $s^{\mathbb{Z}}$ of the moduli space $\mathcal{M}_{k+1}^{\operatorname{main}}\left(\beta ; P_{1}, \ldots, P_{k}\right)$ to define $\mathfrak{m}_{k}^{\mathbb{Z}}$. We also took (in [6]) a 
transversal multisection $s^{\mathbb{Q}}$ of the same moduli space to define $\mathfrak{m}_{k}^{\mathbb{Q}}$. We will find a perturbation of (95) which interpolates these two perturbations.

Definition 14.4. A strongly piecewise smooth multisection $s$ on a space with Kuranishi structure $X$ is said to be a weakly transversal and weakly normally conical multisection, if the following condition is satisfied. We decompose

$$
s=\bigoplus_{i} s^{\Gamma_{i}} \oplus s^{\Gamma} \oplus s^{\perp}
$$

according to the decomposition (40) (41).

(1) Each of $s^{\Gamma}$ is of general position to 0 on $X^{\cong}(\Gamma)$.

(2) Instead of (48) the following equality is satisfied.

$$
\begin{aligned}
& s(p)=s^{\Gamma}\left(\pi_{\Gamma}(p)\right) \\
& +\sum_{\Gamma^{\prime} \subsetneq \Gamma}( \\
& \quad\left(1-\exp \left(\frac{1}{d^{2}}-\frac{1}{\rho_{\Gamma}(p)}\right)\right) s^{\Gamma^{\prime}}\left(\pi_{\Gamma}(p)\right) \\
& \left.\quad+\exp \left(\frac{1}{d^{2}}-\frac{1}{\rho_{\Gamma}(p)}\right) s^{\Gamma^{\prime}}\left(r_{\Gamma}(d)(p)\right)\right) .
\end{aligned}
$$

(Note for each branch of $s^{\Gamma^{\prime}}\left(\pi_{\Gamma}(p)\right)$ there is a branch of $s^{\Gamma^{\prime}}\left(r_{\Gamma}(d)(p)\right)$. We take the sum branch-wise in (96).)

We note that if $s$ is a single valued section then $s^{\Gamma^{\prime}}\left(\pi_{\Gamma}(p)\right)$ is automatically 0 . (Note $s^{\Gamma^{\prime}}$ is a component of $s^{\perp}$.) So (96) coincides with (48) in that case. When $s$ is a multisection $s^{\Gamma^{\prime}}\left(\Gamma^{\prime} \subsetneq \Gamma\right)$ may not be 0 on $X^{\cong}(\Gamma)$.

We also note that we may choose our multisection $s^{\mathbb{Q}}$ so that it is weakly transversal and weakly normally conical. In fact, we may take $\left(s^{\mathbb{Q}}\right)^{\Gamma^{\prime}}$ to be locally constant in the normal direction. Namely

$$
\left(s^{\mathbb{Q}}\right)^{\Gamma^{\prime}}\left(\pi_{\Gamma}(p)\right)=\left(s^{\mathbb{Q}}\right)^{\Gamma^{\prime}}\left(r_{\Gamma}(d)(p)\right) .
$$

Now in the same way as in Section 13 we can construct a system of weakly transversal and weakly normally conical multisections $s$ on (95) such that it is compatible with $s^{\mathbb{Z}}$ on the component of time allocation 0 and with $s^{\mathbb{Q}}$ on the component of time allocation 1.

We may choose $s$ so that $s^{-1}(0)$ has a triangulation, in the following way.

(1) As for the component of the obstruction bundle coming from the component with time allocation 0 , we assume $s^{\perp}=0$. 
(2) For other components, we assume $s$ is transversal to zero.

(3) In the tubular neighborhood of the stratum of the moduli space defined by the equation that time allocation $=0$, the section $s^{\perp}$ is normally conical.

We explain Item (1) above more precisely. Let $p=\left(\Sigma, \vec{z}, u,\left\{\rho_{a}\right\}\right)$ represent an element of $\mathcal{M}_{k+1}^{\text {main }}\left(\{J\}_{\rho}: \beta\right.$; $\left.\operatorname{top}(\rho)\right)$, where $(\Sigma, \vec{z})$ is a bordered marked Riemann surface, $u:(\Sigma, \partial \Sigma) \rightarrow(M, L)$ and $\left\{\rho_{a}\right\}$ is a time allocation. The fiber $E_{p}$ of the obstruction bundle is a finite dimensional subspace of $C^{\infty}\left(\Sigma ; u^{*} T M \otimes \Lambda^{0,1}\right)$, the space of the smooth sections of the vector bundle $u^{*} T M \otimes \Lambda^{0,1}$. (See [7] proof of Proposition 7.1.12.) The support of elements of $E_{p}$ is disjoint from the singular points. And $E_{p}$ is decomposed to the direct sum $\bigoplus_{a}\left(E_{p}\right)_{a}$, where $\Sigma=\cup_{a} \Sigma_{a}$ is a decomposition to the components and elements of $\left(E_{p}\right)_{a}$ are supported on $\Sigma_{a}$. The consistency with $s^{\mathbb{Z}}$ requires us to set the component of $s^{\perp}$ in $\left(E_{p}\right)_{a}$ with $\rho_{a}=0$ is zero. This is Item (1) above.

We can construct such a multisection by the inducition of the stratum in the same way as in Section 9. It is easy to see that $s^{-1}(0)$ satisfies the conclusion of Theorem 3.1. Therefore, we can use it in the same way as (90) to define the required homotopy equivalence. The proof of Lemma 14.2 is complete.

The proof of Theorem 1.1 is now complete.

The proof of Theorem 1.2 over $\mathbb{Z}$ is again similar and parallel to [6], [7]. So we omit it.

\section{How the Results of [6], [7] ARE Generalized to $\mathbb{Z}$ or $\mathbb{Z}_{2}$ COEFFICIENTS}

15.1. Statements. Throughout this section we always assume $(M, \omega)$ is a spherically positive symplectic manifold. We assume its Lagrangian submanifold $L$ is compact, oriented and relatively spin when the ground ring $R$ is a Dedekind domain (for example $\mathbb{Z}$ ) or is a finite field of odd characteristic (for example $\left.\mathbb{Z}_{p}=\mathbb{Z} / p \mathbb{Z}(p \neq 2)\right)$. In case when $R$ is a finite field of even characteristic (for example $\mathbb{Z}_{2}$ ) we only assume $L$ to be a compact Lagrangian submanifold.

The various structures we mention in this section depend on the connected

component of $\mathcal{J}_{(M, \omega)}^{c_{1}>0}$. The various well-definedness or functoriality statement should be understood in the same way as in Theorem 1.1 (2). 
The following is a version of Theorem B [6]. Theorem 15.1 follows from Theorem 1.1 by a purely algebraic argument.

Theorem 15.1. We can associate a set $\mathcal{M}_{\text {weak }}(L ; R)$ and a map $\mathfrak{P O}$ : $\mathcal{M}_{\text {weak }}(L ; R) \rightarrow \Lambda_{0, \text { nov }}^{+, R}$, which have the following properties

(1) There is a Floer cohomology $\operatorname{HF}\left(\left(L, b_{1}\right),\left(L, b_{0}\right) ; \Lambda_{0, \text { nov }}^{R}\right)$ parameterized by

$$
\left(b_{1}, b_{0}\right) \in \mathcal{M}_{\text {weak }}(L ; R) \times_{\mathfrak{P O}} \mathcal{M}_{\text {weak }}(L ; R) .
$$

Here $\mathcal{M}_{\text {weak }}(L ; R) \times_{\mathfrak{P O}} \mathcal{M}_{\text {weak }}(L ; R)$ is the set of pairs $\left(b_{1}, b_{0}\right) \in$ $\mathcal{M}_{\text {weak }}(L ; R) \times \mathcal{M}_{\text {weak }}(L ; R)$ such that $\mathfrak{P O}\left(b_{0}\right)=\mathfrak{P O}\left(b_{1}\right)$.

(2) There exists a product structure

$$
\begin{aligned}
\mathfrak{m}_{2}: H F\left(\left(L, b_{2}\right),\left(L, b_{1}\right) ; \Lambda_{0, \text { nov }}^{R}\right) & \otimes H F\left(\left(L, b_{1}\right),\left(L, b_{0}\right) ; \Lambda_{0, \text { nov }}^{R}\right) \\
& \rightarrow H F\left(\left(L, b_{2}\right),\left(L, b_{0}\right) ; \Lambda_{0, \text { nov }}^{R}\right)
\end{aligned}
$$

if $\left(b_{1}, b_{0}\right),\left(b_{2}, b_{1}\right) \in \mathcal{M}_{\text {weak }}(L ; R) \times_{\mathfrak{P O}} \mathcal{M}_{\text {weak }}(L ; R)$. The product $\mathfrak{m}_{2}$ is associative. In particular, $\operatorname{HF}\left((L, b),(L, b) ; \Lambda_{0, \text { nov }}^{R}\right)$ has the ring structure for any $b \in \mathcal{M}_{\text {weak }}(L ; R)$.

(3) If $\psi:(M, L) \rightarrow\left(M^{\prime}, L^{\prime}\right)$ is a symplectic diffeomorphism, then it induces a bijection $\psi_{*}: \mathcal{M}_{\text {weak }}(L ; R) \rightarrow \mathcal{M}_{\text {weak }}\left(L^{\prime} ; R\right)$ such that $\mathfrak{P O} \circ \psi_{*}=\mathfrak{P O}$. The map $\psi_{*}$ depends only on the isotopy class of symplectic diffeomorphism $\psi$.

(4) In the situation of (3), we have an isomorphism

$$
\psi_{*}: H F\left(\left(L, b_{1}\right),\left(L, b_{0}\right) ; \Lambda_{0, \text { nov }}^{R}\right) \cong H F\left(\left(L^{\prime}, \psi_{*}\left(b_{1}\right)\right),\left(L^{\prime}, \psi_{*}\left(b_{0}\right)\right) ; \Lambda_{0, \text { nov }}^{R}\right),
$$

and $\psi_{*}$ commutes with $\mathfrak{m}_{2}$.

(5) The isomorphism $\psi_{*}$ in (4) depends only on the isotopy class of symplectic diffeomorphism $\psi:(M, L) \rightarrow\left(M^{\prime}, L^{\prime}\right)$. Moreover $\left(\psi \circ \psi^{\prime}\right)_{*}=\psi_{*} \circ \psi_{*}^{\prime}$.

Remark 15.1. (1) We do not include bulk deformations in Theorem 15.1. Namely we consider $\mathcal{M}_{\text {weak }}(L ; R)$ and not $\mathcal{M}_{\text {weak,def }}(L ; R)$. Including bulk deformations is a bit difficult to work out in our case for coefficients in a Dedekind domain or a finite field. For example, we used homotopy theory of filtered $L_{\infty}$ algebra in [7] Section 7.4, for the algebraic formulation of bulk deformations and of the operator $\mathfrak{q}$. Homotopy theory of filtered $L_{\infty}$ algebra is hard to study over torsion coefficients. However, it seems very likely that we can go around this problem and define $\mathcal{M}_{\text {weak,def }}(L ; R)$ and Floer cohomology parametrized by it over a 
Dedekind domain or a finite field coefficients. We postpone it to future research.

(2) The Maurer-Cartan moduli space $\mathcal{M}_{\text {weak }}(L ; R)$ in Theorem 15.1 is one over $\Lambda_{0, \text { nov }}^{+, R}$. Namely it is the set of the gauge equivalence classes of the chains $b \in S\left(L ; \Lambda_{0, \text { nov }}^{+, R}\right)$ satisfying the equation

$$
\sum_{k=0}^{\infty} \mathfrak{m}_{k}\left(b^{k}\right) \equiv 0 \quad \bmod \Lambda_{0, \text { nov }}^{+, R} \mathbf{e}
$$

where $\mathbf{e}$ is the unit. (See [6] Section 4.3.) In $[10,11,5]$ we enhanced it to one over $\Lambda_{0, \text { nov }}^{R}$ coefficients in case $R=\mathbb{R}$ or $R=\mathbb{C}$. We will discuss this enhancement in the case when $R$ is a Dedekind domain or a finite field in Subsection 15.3.

The next result is a version of Theorem $\mathrm{C}$ [6]. The proof is the same as that of Theorem C [6] by using Theorem 1.1.

Theorem 15.2. There exists a series of positive integers $m_{k}<\operatorname{dim} L / 2$ and classes

$$
\left[o_{k}^{2 m_{k}}(L ; \text { weak })\right] \in H^{2 m_{k}}(L ; R)
$$

$k=1,2, \ldots$, such that, if $\left[o_{k}^{2 m_{k}}(L\right.$; weak $\left.)\right]$ are all zero, then $\mathcal{M}_{\text {weak }}(L ; R)$ is nonempty. The number $2-2 m_{k}$ is a sum of the Maslov indices of a finite collection of the homotopy classes in $\pi_{2}(M, L)$ realized by pseudo-holomorphic discs (with respect to a given almost complex structure on $M$ ).

Next, we consider analogs of Theorem D and Theorem E [6] on the spectral sequence. Because of convergence issue of the spectral sequence over $\Lambda_{0 \text {,nov }}^{R}$ we consider the following assumption.

Assumption 15.2. We assume one of the following conditions.

(1) $R$ is a finite field of characteristic 2 .

(2) $R$ is a finite field of odd characteristic and $L$ is relatively spin.

(3) $R$ is a Dedekind domain, $L$ is relatively spin and rational in the sense of [6] Definition 6.2.1. In this case, Theorem 15.3 only applies to $b_{1}, b_{0}$ of the form $\sum T^{\lambda_{i}} e^{\mu_{i} / 2} b_{i}$ such that the subgroup of $\mathbb{R}$ generated by the set $\left\{\lambda_{i}\right\} \cup\left\{\beta(\omega) \mid \beta \in \pi_{2}(M, L)\right\}$ is isomorphic to $\mathbb{Z}$. 
We denote by $\left(\Lambda_{0, \text { nov }}^{R}\right)^{(p)}$ the degree $p$ part of $\Lambda_{0, \text { nov }}^{R}$, where we recall $\operatorname{deg}\left(a T^{\lambda} e^{\mu}\right)=2 \mu$ for $a T^{\lambda} e^{\mu} \in \Lambda_{\text {nov }}^{R}$.

Theorem 15.3. Under Assumption 15.2 there exists a spectral sequence for each $\left(b_{1}, b_{0}\right) \in \mathcal{M}_{\text {weak }}(L ; R) \times \mathfrak{P O} \mathcal{M}_{\text {weak }}(L ; R)$ with the following properties:

(1) $E_{2}^{p, q}=\bigoplus_{k} H^{k}(L ; R) \otimes\left(T^{q \lambda} \Lambda_{0, \text { nov }}^{R} / T^{(q+1) \lambda} \Lambda_{0, \text { nov }}^{R}\right)^{(p-k)}$. Here $\lambda>0$.

(2) There exists a filtration $\mathfrak{F}^{*} H F\left(\left(L, b_{1}\right),\left(L, b_{0}\right) ; \Lambda_{0, \text { nov }}^{R}\right)$ on the Floer cohomology $H F\left(\left(L, b_{1}\right),\left(L, b_{0}\right) ; \Lambda_{0, \text { nov }}^{R}\right)$ such that

$$
E_{\infty}^{p, q} \cong \frac{\mathfrak{F}^{q} H F^{p}\left(\left(L, b_{1}\right),\left(L, b_{0}\right) ; \Lambda_{0, \text { nov }}^{R}\right)}{\mathfrak{F}^{q+1} H F^{p}\left(\left(L, b_{1}\right),\left(L, b_{0}\right) ; \Lambda_{0, \text { nov }}^{R}\right)} .
$$

(3) Consider the subgroup $K_{r} \subset E_{r}=\bigoplus_{p, q} E_{r}^{p, q}$ defined by

$$
\begin{aligned}
K_{2}^{p, q}= & \bigoplus_{k} P D\left(\operatorname{Ker}\left(H_{n-k}(L ; R) \rightarrow H_{n-k}(M ; R)\right)\right) \\
& \otimes\left(T^{q \lambda} \Lambda_{0, \text { nov }}^{R} / T^{(q+1) \lambda} \Lambda_{0, \text { nov }}^{R}\right)^{(p-k)}, \\
K_{r+1}= & \frac{K_{r} \cap \operatorname{Ker} \delta_{r}}{K_{r} \cap \operatorname{Im} \delta_{r}} \subset E_{r+1} .
\end{aligned}
$$

Then we have $\operatorname{Im} \delta_{r} \subseteq K_{r}$ for every $r$, under the additional assumption that $b_{0}=b_{1}$.

In particular, if the homomorphism $i_{*}: H_{*}(L ; R) \rightarrow H_{*}(M ; R)$ induced by the inclusion is injective, the spectral sequence collapses at the $E_{2}$ level.

(4) The spectral sequence is compatible with the ring structure in Theorem 15.1 (2). In other words, we have the following. Each of $E_{r}$ has a ring structure $\mathfrak{m}_{2}$ which satisfies:

$$
\delta_{r}\left(\mathfrak{m}_{2}(x, y)\right)=-\mathfrak{m}_{2}\left(\delta_{r}(x), y\right)+(-1)^{\operatorname{deg} x} \mathfrak{m}_{2}\left(x, \delta_{r}(y)\right) .
$$

The filtration $\mathfrak{F}$ is compatible with the ring structure in Theorem 15.1 (2). The isomorphisms in Theorem 15.3 (1),(2) are ring isomorphisms.

By using Theorem 1.1, the proof of Theorem 15.3 is the same as that of Theorem D [6], except the following two points. Assumption 15.2 is used in a way described in (1) below.

(1) During the construction of the spectral sequence, we used the algebraic material given in [6] Subsections 6.3.1 and 6.3.2. We assumed that the ground ring $R$ is a field there. (The authors do not know how to prove 
the convergence of the spectral sequence in the case $R=\mathbb{Z}$.) In the case $L, b_{0}, b_{1}$ satisfy Assumption $15.2(2)$, we can use the argument of [6] Section 6.2 and can construct the spectral sequence and prove its convergence in the case when $R$ is a Dedekind domain also.

(2) In [6] we used the operator $\mathfrak{p}$ and the cyclic cohomology of $L$ to prove a statement (3) of degeneration of the spectral sequence over $\mathbb{Q}([6]$ Subsection 6.4.2). It seems hard to study cyclic cohomology over torsion coefficients. However in Subsection 15.4 we will use the Hochschild homology instead, and introduce an analogous operator $\mathfrak{p}^{\prime}$ to prove (3) over $R$.

The following non-vanishing theorem is an analog of Theorem E [6] and the proof is the same except that of $P D[p t] \notin \operatorname{Im}\left(\delta_{r}\right)$ in (1), where we used the operator $\mathfrak{p}$ for the case over $\mathbb{Q}$ in $[6]$ Subsection 6.4.3. We will also use the analogous operator $\mathfrak{p}^{\prime}$ over $R$. See Subsection 15.4 for this point.

Theorem 15.4. Let us consider the situation of Theorem 15.3. We assume $b_{0}=b_{1}$. Then, there exists a cohomology class $P D[L]^{\prime} \in H^{n}\left(L ; \Lambda_{0, \text { nov }}^{R}\right)$ with $P D[L]^{\prime} \equiv P D[L] \bmod \Lambda_{0, \text { nov }}^{+, R}$ which has the following properties.

(1) For each $r$ we have $\delta_{r}\left(P D[L]^{\prime}\right)=0$ and $P D[p t] \notin \operatorname{Im}\left(\delta_{r}\right)$, where $\delta_{r}$ is the differential of the spectral sequence in Theorem 15.3.

(2) If the Maslov index of all the pseudo-holomorphic discs bounding $L$ are non-positive, then $\delta_{r}(P D[p t])=0$ and $P D[L]^{\prime} \notin \operatorname{Im}\left(\delta_{r}\right)$.

The same conclusion holds for $\Lambda_{\text {nov }}^{R}$ coefficients.

The next theorem is an analog of Theorem G [6] and follows from Theorem 1.2 .

Theorem 15.5. Let $\left(L_{1}, L_{0}\right)$ be a pair of Lagrangian submanifolds of $M$ of clean intersection. Then, for each $\left(b_{1}, b_{0}\right) \in \mathcal{M}_{\text {weak }}\left(L_{1} ; R\right) \times_{\mathfrak{P O}} \mathcal{M}_{\text {weak }}\left(L_{0} ; R\right)$, we can associate a Floer cohomology $H F\left(\left(L_{1}, b_{1}\right),\left(L_{0}, b_{0}\right) ; \Lambda_{0, \text { nov }}^{R}\right)$ with the following properties. We put

$$
H F\left(\left(L_{1}, b_{1}\right),\left(L_{0}, b_{0}\right) ; \Lambda_{0, \text { nov }}^{R}\right) \otimes_{\Lambda_{0, \text { nov }}^{R}} \Lambda_{\text {nov }}^{R}=H F\left(\left(L_{1}, b_{1}\right),\left(L_{0}, b_{0}\right) ; \Lambda_{\text {nov }}^{R}\right) .
$$

(1) If $L_{0}=L_{1}=L$, then $\operatorname{HF}\left(\left(L_{1}, b_{1}\right),\left(L_{0}, b_{0}\right) ; \Lambda_{0, \text { nov }}^{R}\right)$ coincides with the one in Theorem 15.1 . 
(2) If $R$ is a field, then we have

$$
\operatorname{rank}_{\Lambda_{\text {nov }}^{R}} H F\left(\left(L_{1}, b_{1}\right),\left(L_{0}, b_{0}\right) ; \Lambda_{\text {nov }}^{R}\right) \leq \sum_{h, k} \operatorname{rank}_{R} H^{k}\left(R_{h} ; \Theta_{R_{h}}^{-}\right),
$$

where each $R_{h}$ is a connected component of $L_{0} \cap L_{1}$ and $\Theta_{R_{h}}^{-}$is a local system on it.

(3) If $\psi: M \rightarrow M^{\prime}$ is a symplectic diffeomorphism with $\psi\left(L_{i}\right)=L_{i}^{\prime},(i=0,1)$, then we have a canonical isomorphism

$$
\psi_{*}: H F\left(\left(L_{1}, b_{1}\right),\left(L_{0}, b_{0}\right) ; \Lambda_{0, \text { nov }}^{R}\right) \cong H F\left(\left(L_{1}^{\prime}, \psi_{*} b_{1}\right),\left(L_{0}^{\prime}, \psi_{*} b_{0}\right) ; \Lambda_{0, \text { nov }}^{R}\right)
$$

where $\psi_{*}: \mathcal{M}_{\text {weak }}\left(L_{i} ; R\right) \rightarrow \mathcal{M}_{\text {weak }}\left(L_{i}^{\prime} ; R\right)$ is as in Theorem 15.1. The isomorphism $\psi_{*}$ depends only on the isotopy class of symplectic diffeomorphism $\psi$ with $\psi\left(L_{i}\right)=L_{i}^{\prime}$. We also have $\left(\psi \circ \psi^{\prime}\right)_{*}=\psi_{*} \circ \psi_{*}^{\prime}$.

(4) If $\psi_{i}^{s}: M \rightarrow M(i=0,1, s \in[0,1])$ are Hamiltonian isotopies with $\psi_{i}^{0}=i d e n t i t y$ and $\psi_{i}^{1}\left(L_{i}\right)=L_{i}^{\prime}$, then it induces an isomorphism

$$
\left(\psi_{1}^{s}, \psi_{0}^{s}\right)_{*}: H F\left(\left(L_{1}, b_{1}\right),\left(L_{0}, b_{0}\right) ; \Lambda_{\text {nov }}^{R}\right) \cong H F\left(\left(L_{1}^{\prime}, \psi_{1 *}^{1} b_{1}\right),\left(L_{0}^{\prime}, \psi_{0 *}^{1} b_{0}\right) ; \Lambda_{\text {nov }}^{R}\right) .
$$

The isomorphism $\left(\psi_{1}^{s}, \psi_{0}^{s}\right)_{*}$ depends only the isotopy class of the Hamiltonian isotopies $\psi_{i}^{s}: M \rightarrow M(i=0,1, s \in[0,1])$ with $\psi_{i}^{0}=i d e n t i t y$ and $\psi_{i}^{1}\left(L_{i}\right)=L_{i}^{\prime}$. The isomorphism $\left(\psi_{1}^{s}, \psi_{0}^{s}\right)_{*}$ is functorial with respect to the composition of the Hamiltonian isotopies.

(5) $\operatorname{HF}\left(\left(L_{1}, b_{1}\right),\left(L_{0}, b_{0}\right) ; \Lambda_{0, \text { nov }}^{R}\right)$ is a bimodule over the ring pair

$$
\left(H F\left(\left(L_{1}, b_{1}\right),\left(L_{1}, b_{1}\right) ; \Lambda_{0, \text { nov }}^{R}\right), H F\left(\left(L_{0}, b_{0}\right),\left(L_{0}, b_{0}\right) ; \Lambda_{0, \text { nov }}^{R}\right)\right) \text {. }
$$

The isomorphisms (3), (4) are bimodule isomorphisms.

We state Theorem 15.5 only in the case when $\left(L_{1}, L_{0}\right)$ has clean intersection. In the case when $R$ is a finite field, we can use a version of Theorem 6.1.25 [6] in the same way as in [6] Subsection 6.5.4 to remove this assumption over $\Lambda_{0, \text { nov }}^{R}$ coefficients.

[6] Theorem I can be generalized as follows.

Theorem 15.6. Under Assumption 15.2 (1) or (2), we assume that $\mathcal{M}_{\text {weak }}(L ; R)$ is non-empty. Denote $A=\sum_{*} \operatorname{rank} H_{*}(L ; R), B=\sum_{*} \operatorname{rank} \operatorname{Ker}\left(H_{*}(L ; R) \rightarrow\right.$ $\left.H_{*}(M ; R)\right)$. Then we have

$$
\#(L \cap \phi(L)) \geq A-2 B
$$


for any Hamiltonian diffeomorphism $\phi: M \rightarrow M$ such that $L \pitchfork \phi(L)$.

The proof is the same as that of [6] Theorem I using Theorems 15.3 and 15.5.

Theorem J [6] is generalized as follows. We consider the case when Assumption 15.2 (1) or (2) is satisfied. Let $\phi$ be a Hamiltonian diffeomorphism. We assume $\phi\left(L_{1}\right)$ is transverse to $L_{0}$ and $b_{i} \in \mathcal{M}_{\text {weak }}\left(L_{i} ; R\right)$ with $\mathfrak{P O}\left(b_{0}\right)=\mathfrak{P O}\left(b_{1}\right)$. Since the algebraic argument in Subsections 6.3 .1 and 6.3.2 [6] works over an arbitrary field $R$, we can prove

$$
H F\left(\left(L_{1}, b_{1}\right),\left(L_{0}, b_{0}\right) ; \Lambda_{0, \text { nov }}^{R}\right) \cong\left(\Lambda_{0, \text { nov }}^{R}\right) \oplus a \bigoplus_{i=1}^{b}\left(\Lambda_{0, \text { nov }}^{R} / T^{\lambda_{i}} \Lambda_{0, \text { nov }}^{R}\right)
$$

in the same way as in [6] Theorem 6.1.18 (and also Theorem 6.1.20). We call $a$ the Betti number and $\lambda_{i}$ torsion exponents.

Theorem 15.7. Under Assumption 15.2 (1) or (2), we denote

$$
b(\|\phi\|)=\#\left\{i \mid \lambda_{i} \geq\|\phi\|\right\},
$$

where $\lambda_{i}$ are the torsion exponents as in (98) and $\|\phi\|$ is the Hofer norm. Then we have

$$
\#\left(\phi\left(L_{1}\right) \cap L_{0}\right) \geq a+2 b(\|\phi\|) .
$$

The original proof of Theorem $\mathrm{J}$ [6] contains an error related to an energy estimate. See [13]. We now have corrected it in [13]. Then the proof of Theorem 15.7 is the same as that of Theorem J given in [13]. Theorem 6.1.25 [6] can be generalized in the same way.

\subsection{Proof of Theorems $\mathbf{1 . 3}$ and $\mathbf{1 . 4}$.}

Proof of Theorem 1.3. We prove Theorem 1.3 by contradiction. Suppose that the Maslov class vanishes. Theorem 15.2 and the assumption imply that all the obstruction classes $\left[o_{k}^{2 m_{k}}(L\right.$; weak $\left.)\right]$ are in $H^{2}\left(L ; \mathbb{Z}_{2}\right)$. Therefore by assumption that $H^{2}\left(L ; \mathbb{Z}_{2}\right)=0$, we have $b$ such that the Floer cohomology $H\left((L, b),(L, b) ; \Lambda_{0, \text { nov }}^{\mathbb{Z}_{2}}\right)$ is defined.

By Theorem $15.4(1)$ we have $\delta_{r}\left(P D[L]^{\prime}\right)=0$. Moreover by Theorem 15.4 (2) $P D[L]^{\prime} \notin \operatorname{Im} \delta_{r}$. Thus we have $H\left((L, b),(L, b) ; \Lambda_{\text {nov }}^{\mathbb{Z}_{2}}\right) \neq 0$. Then Theorem 15.5 (1),(4) imply that

$$
H\left((L, b),\left(\psi(L), \psi_{*} b\right) ; \Lambda_{\text {nov }}^{\mathbb{Z}_{2}}\right) \neq 0
$$


for any Hamiltonian diffeomorphism $\psi$. Theorem 15.5 (2) now implies

$$
L \cap \psi(L) \neq \emptyset
$$

which leads to a contradiction.

Theorem 1.4 follows easily from Theorem 1.3.

15.3. Maurer-Cartan moduli space over $\Lambda_{0, \text { nov }}^{R}$. We considered the filtered $A_{\infty}$ algebra $\left(S\left(L ; \Lambda_{0, \text { nov }}^{R}\right),\left\{\mathfrak{m}_{k}\right\}\right)$ in Sections 13 and 14 . For $b \in S^{1}\left(L ; \Lambda_{0, \text { nov }}^{+, R}\right)$ we define a deformation of filtered $A_{\infty}$ structure by

$$
\mathfrak{m}_{k}^{b}\left(x_{1}, \ldots, x_{k}\right)=\sum_{\ell_{0}=0}^{\infty} \cdots \sum_{\ell_{k}=0}^{\infty} \mathfrak{m}_{k+\sum \ell_{i}}\left(b^{\ell_{0}}, x_{1}, b^{\ell_{1}}, \ldots, x_{k}, b^{\ell_{k}}\right) .
$$

Using the fact that $b \equiv 0 \bmod \Lambda_{0, \text { nov }}^{+, R}$ we can prove that the right hand side of (100) converges in $T$ adic topology. The Maurer-Cartan equation (97) becomes $\mathfrak{m}_{0}^{b} \equiv 0 \bmod \Lambda_{0, \text { nov }}^{+, R} \mathbf{e}$.

In the case $R=\mathbb{R}$ (resp. $\mathbb{C}$ ), this story is generalized in $[10,11,5]$ to the case when $b \in S^{1}\left(L ; \Lambda_{0, \text { nov }}^{\mathbb{R}}\right)$ (resp. $\left.b \in S^{1}\left(L ; \Lambda_{0, \text { nov }}^{\mathbb{C}}\right)\right)$ as follows. We recall the case $R=\mathbb{R}$ only, because the case $R=\mathbb{C}$ is similar. We put

$$
b=\bar{b}_{1}+b_{+}
$$

where

$$
\bar{b}_{1} \in S^{1}(L ; \mathbb{R}), \quad b_{+} \in S^{1}\left(L ; \Lambda_{0, \text { nov }}^{(0)+, \mathbb{R}}\right) \oplus \bigoplus_{k \geq 1} S^{1+2 k}\left(L ; \Lambda_{0, \text { nov }}^{(0) \mathbb{R}}\right) e^{-k} .
$$

Here $\Lambda_{0, \text { nov }}^{(0) R}$ is the degree 0 part of $\Lambda_{0, \text { nov }}^{R}$, namely, the part which does not contain the indeterminate $e$. We assume $\partial \bar{b}_{1}=0$, where $\partial$ is the usual boundary operator. (This will follow from the Maurer-Cartan equation.) We decompose $\mathfrak{m}_{k}$ as

$$
\mathfrak{m}_{k}=\sum_{\beta \in \Pi_{2}(M, L)} T^{\omega(\beta)} e^{\mu(\beta) / 2} \mathfrak{m}_{k, \beta} .
$$

In the situation of $[10,11,5]$ we take the moduli space $\mathcal{M}_{k+1}(\beta)$ and its perturbation so that it is compatible with the forgetful map of the 1st, $\ldots, k$-th marked points $\mathcal{M}_{k+1}(\beta) \rightarrow \mathcal{M}_{1}(\beta)$. (See [5] Section 5 for the precise description of the compatibility.) We used this fact to show the following formula for $\bar{b}_{1} \in S^{1}(L ; \mathbb{R})$ :

$$
\sum_{\ell_{0}+\cdots+\ell_{k}=\ell} \mathfrak{m}_{k+\ell, \beta}\left(\bar{b}_{1}^{\ell_{0}}, x_{1}, \ldots, x_{k}, \bar{b}_{1}^{\ell_{k}}\right)=\frac{\left(\bar{b}_{1}(\partial \beta)\right)^{\ell}}{\ell !} \mathfrak{m}_{k, \beta}\left(x_{1}, \ldots, x_{k}\right) .
$$


(See [11] Lemmas 7.2, 9.2 and [5] Lemma 13.1.) Using (101) we define for $b \in$ $S^{1}\left(L ; \Lambda_{0, \text { nov }}^{\mathbb{R}}\right)$

$\mathfrak{m}_{k}^{b}\left(x_{1}, \ldots, x_{k}\right)=\sum_{\beta} \sum_{\ell_{0}, \ldots, \ell_{k}=0}^{\infty} T^{\omega(\beta)} e^{\mu(\beta) / 2} \exp \left(\bar{b}_{1}(\partial \beta)\right) \mathfrak{m}_{k+\sum \ell_{i}, \beta}\left(b_{+}^{\ell_{0}}, x_{1}, \ldots, x_{k}, b_{+}^{\ell_{k}}\right)$

by modifying the definition (100) for the case $b \in S^{1}\left(L ; \Lambda_{0, \text { nov }}^{+, \mathbb{R}}\right)$. See (11.4) in [11] for toric cases. Using Gromov's compactness we can show that the right hand side converges in $T$ adic topology. (See [11] Section 9 and [5] Lemma 13.3.) This definition is closely related to the idea of [3] to use nonunitary flat bundles on $L$. Namely, in this case we take a flat line bundle with monodromy

$$
\gamma \mapsto \exp \left(\bar{b}_{1}(\gamma)\right)
$$

In order to generalize this story to the case when $R \neq \mathbb{R}, \mathbb{C}$ but $R$ is a Dedekind domain or a finite field, there are two points we need to take care of.

Firstly the compatibility of the perturbation to the forgetful map $\mathcal{M}_{k+1}(\beta) \rightarrow$ $\mathcal{M}_{1}(\beta)$ is hard to prove when we use single valued perturbations. (The proof of [5] does not work.) So instead of proving (101) we use the right hand side as the definition.

Secondly there is a denominator $\ell$ ! in (101) which may not be invertible in $R$. So instead of requiring $\bar{b}_{1} \in R$ we require $\exp \left(\bar{b}_{1}(\partial \beta)\right) \in R$. (Compare [11] Remark 11.5.)

After these explanations we state our results. We conside a pair $\left(\rho, b_{+}\right)$where

$$
\rho \in \operatorname{Hom}\left(\pi_{1}(L), R^{*}\right), \quad b_{+} \in S^{1}\left(L ; \Lambda_{0, \text { nov }}^{(0)+, R}\right) \oplus \bigoplus_{k \geq 1} S^{1+2 k}\left(L ; \Lambda_{0, \text { nov }}^{(0) R}\right) e^{-k} .
$$

Here $R^{*}$ is the group of units of the ring $R$. We put

$$
\widehat{\mathcal{M}}_{\text {weak }}\left(L ; \Lambda_{0, \text { nov }}^{R}\right)=\left\{\left(\rho, b_{+}\right) \mid \sum_{\beta, k} T^{\omega(\beta)} e^{\mu(\beta) / 2} \rho(\partial \beta) \mathfrak{m}_{k, \beta}\left(b_{+}^{k}\right) \equiv 0 \quad \bmod \Lambda_{0, \text { nov }}^{+, R} \mathbf{e}\right\} .
$$

The infinite sum appearing in (103) converges in $T$ adic topology.

Remark 15.3. We may use a flat vector bundle in place of a flat line bundle $\rho$.

We say $\left(\rho, b_{+}\right)$is gauge equivalent to $\left(\rho^{\prime}, b_{+}^{\prime}\right)$ if $\rho$ is conjugate to $\rho^{\prime}$ as representations of $\pi_{1}(L)$ to $R^{*}$ and $b_{+}$is gauge equivalent to $b_{+}^{\prime}$ as Maurer-Cartan 
elements of the filtered $A_{\infty}$ structure $\mathfrak{m}_{k}^{\rho}$ in the sense of [6] Definition 4.3.19, where

$$
\mathfrak{m}_{k}^{\rho}=\sum_{\beta} T^{\omega(\beta)} e^{\mu(\beta) / 2} \rho(\partial \beta) \mathfrak{m}_{k, \beta}
$$

We denote the set of gauge equivalence classes of $\widehat{\mathcal{M}}_{\text {weak }}\left(L ; \Lambda_{0, \text { nov }}^{R}\right)$ by $\mathcal{M}_{\text {weak }}\left(L ; \Lambda_{0, \text { nov }}^{R}\right)$.

Definition 15.4. For $\left(\rho, b_{+}\right) \in \mathcal{M}_{\text {weak }}\left(L ; \Lambda_{0, \text { nov }}^{R}\right)$ we define a deformed filtered $A_{\infty}$ structure $\mathfrak{m}_{k}^{\left(\rho, b_{+}\right)}$by

$\mathfrak{m}_{k}^{\left(\rho, b_{+}\right)}\left(x_{1}, \ldots, x_{k}\right)=\sum_{\beta} \sum_{\ell_{0}, \ldots, \ell_{k}=0}^{\infty} T^{\omega(\beta)} e^{\mu(\beta) / 2} \rho(\partial \beta) \mathfrak{m}_{k+\sum \ell_{i}, \beta}\left(b_{+}^{\ell_{0}}, x_{1}, \ldots, x_{k}, b_{+}^{\ell_{k}}\right)$.

We use it in the same way to show the following:

Theorem 15.8. Theorems 15.1, 15.3, 15.4, 15.5 hold with $\mathcal{M}_{\text {weak }}(L ; R)$ replaced by $\mathcal{M}_{\text {weak }}\left(L ; \Lambda_{0, \text { nov }}^{R}\right)$.

Remark 15.5. We may take the pair $\left(\tilde{\rho}, b_{\text {high }}\right)$ where

$$
\tilde{\rho}: \pi_{1}(L) \rightarrow\left\{y \in \Lambda_{0, \text { nov }}^{R} \mid y \equiv \bar{y} \bmod \Lambda_{0, \text { nov }}^{+, R}, \bar{y} \in R^{*}\right\}
$$

and

$$
b_{\text {high }} \in \bigoplus_{k \geq 1} S^{1+2 k}\left(L ; \Lambda_{0, \text { nov }}^{(0) R}\right) e^{-k}
$$

in place of $\left(\rho, b_{+}\right)$.

In [10] we discussed the case of $T^{n} \cong L \subset X$ where $X$ is a toric manifold and $T^{n}$ is an orbit of the $T^{n}$ action. There we take $b=\sum x_{i} \mathbf{e}_{i}, x_{i} \in \Lambda_{0, \text { nov }}^{\mathbb{C}}$. We then change the coordinate from $x_{i}$ to $y_{i}=\exp \left(x_{i}\right)$. This corresponds to take $\tilde{\rho}$ as above. Thus in case $y_{i}$ is congruent to an element of $R^{*}$ modulo $\Lambda_{0, \text { nov }}^{+, R}$ we can apply the argument of this section. In the toric case, there are many examples where the leading order term of $y_{i}$ is in a number field (a finite extension of $\mathbb{Q}$ ) or its integer ring. (In particular, $y_{i}$ is a unit of its appropriate localization.) For example, in the case $X=\mathbb{C} P^{n}$ nonvanishing Floer cohomology appears when $y_{i}$ is the $(n+1)$-th root of unity. Thus we have many examples for which it seems interesting to study Floer cohomology over $\Lambda_{0, \text { nov }}^{R}$ with a Dedekind domain $R$. 
15.4. Hochschild homology, operator $\mathfrak{p}^{\prime}$ and degeneration of the spectral sequence. In this subsection we prove Theorem 15.3 (3) and the claim $P D[p t] \notin$ $\operatorname{Im}\left(\delta_{r}\right)$ in Theorem 15.4 (1). In their proofs over $\mathbb{Q}$ coefficients given in [6], we used the operator $\mathfrak{p}$ whose domain is the cyclic homology of $L$. See Subsections 3.8.1 and 6.4.2 in [6]. Since it is rather difficult to study cyclic homology over torsion coefficients, we use the Hochschild homology instead in this subsection.

Let $\left(C,\left\{\mathfrak{m}_{k}\right\}\right)$ be a filtered $A_{\infty}$ algebra. We put

$$
C H(C[1])=\widehat{\bigoplus}_{k=0}^{\infty} B_{k+1}(C[1])
$$

We define the Hochschild differential $\delta^{H}$ on it by

$$
\begin{aligned}
& \delta^{H}\left(x_{0} \otimes \cdots \otimes x_{k}\right) \\
= & \sum_{0<i \leq j \leq k}(-1)^{*_{1 ; i}} x_{0} \otimes \cdots \otimes x_{i-1} \otimes \mathfrak{m}_{j-i}\left(x_{i}, \ldots, x_{j-1}\right) \otimes x_{j} \otimes \cdots \otimes x_{k} \\
& +\sum_{i=0}^{k}(-1)^{*_{1 ; i+1}} x_{0} \otimes \cdots \otimes x_{i} \otimes \mathfrak{m}_{k-i}\left(x_{i+1}, \ldots, x_{k}\right) \\
& +\sum_{0 \leq i<j<k}(-1)^{*_{2 ; i, j}} \mathfrak{m}_{k+i-j+1}\left(x_{j+1}, \ldots, x_{k}, x_{0}, \ldots, x_{i}\right) \otimes x_{i+1} \otimes \cdots \otimes x_{j} \\
& +\sum_{i=0}^{k} \mathfrak{m}_{i+1}\left(x_{0}, \ldots, x_{i}\right) \otimes x_{i+1} \otimes \cdots \otimes x_{k},
\end{aligned}
$$

where $*_{1 ; i}=\operatorname{deg} x_{0}+\cdots+\operatorname{deg} x_{i-1}+i, *_{2 ; i, j}=\left(\operatorname{deg} x_{0}+\cdots+\operatorname{deg} x_{j}+j+\right.$ $1)\left(\operatorname{deg} x_{j+1}+\cdots+\operatorname{deg} x_{k}+k-j\right)$. (Note the sum of terms containing $\mathfrak{m}_{0}$ is $\pm x_{0} \otimes \mathfrak{m}_{0}(1) \otimes x_{1} \otimes \cdots \otimes x_{k} \pm \cdots \pm x_{0} \otimes \cdots \otimes x_{k} \otimes \mathfrak{m}_{0}(1)$, each term of which comes from the first and second lines of (105). ) It is straightfoward to check

$$
\delta^{H} \circ \delta^{H}=0 .
$$

We thus obtain a chain complex $\left(C H(C[1]), \delta^{H}\right)$.

We next define a chain map

$$
\mathfrak{p}^{\prime}:\left(C H\left(S\left(L ; \Lambda_{0, \text { nov }}^{R}\right)[1]\right), \delta^{H}\right) \rightarrow S\left(M ; \Lambda_{0, \text { nov }}^{R}\right) .
$$

We use the moduli space $\mathcal{M}_{k ; 1}(\beta)$ which we defined in Section 11. (It is the moduli space of pseudo-holomorphic discs of homology class $\beta$ with $k$ boundary and 1 interior marked points.) We have evaluation maps

$$
\left(e v, e v^{+}\right)=\left(e v_{1}, \ldots, e v_{k}, e v^{+}\right): \mathcal{M}_{k ; 1}(\beta) \rightarrow L^{k} \times M
$$


The boundary of the moduli space is described as follows.

$$
\begin{aligned}
\partial \mathcal{M}_{k ; 1}(\beta)= & \bigcup_{\beta_{1}+\beta_{2}+\beta} \bigcup_{k_{1}+k_{2}=k} \bigcup \mathcal{M}_{k_{1}+1}\left(\beta_{1}\right)_{e v_{0}} \times_{e v_{i}} \mathcal{M}_{k_{2}+1 ; 1}\left(\beta_{2}\right) \\
& \cup \bigcup_{\beta_{1}+\beta_{2}+\beta} \bigcup_{k_{1}+k_{2}=k} \bigcup \mathcal{M}_{k_{1}+1 ; 1}\left(\beta_{1}\right)_{e v_{0}} \times_{e v_{i}} \mathcal{M}_{k_{2}+1}\left(\beta_{2}\right) . k_{2}
\end{aligned}
$$

Let $P_{1}, \ldots, P_{k}$ be smooth singular chains on $L$. We put

$$
\mathcal{M}_{k ; 1}\left(\beta ; P_{1}, \ldots, P_{k}\right)=\mathcal{M}_{k ; 1}(\beta)_{e v} \times\left(P_{1} \times \cdots \times P_{k}\right) .
$$

In Sections 13 and 14 we took a global section of

$$
\mathcal{M}_{k+1}\left(\beta ; P_{1}, \ldots, P_{k}\right)=\mathcal{M}_{k+1}(\beta)_{\left(e v_{1}, \ldots, e v_{k}\right)} \times\left(P_{1} \times \cdots \times P_{k}\right),
$$

which is normally conical. Then we took a triangulation of its zero set so that the virtual fundamental chain is defined. (We use our assumption that $(M, \omega)$ is spherically positivity here.) Now we have:

Lemma 15.9. We can take a global section of $\mathcal{M}_{k ; 1}\left(\beta ; P_{1}, \ldots, P_{k}\right)$ which satisfies the conclusion of Theorem 3.1 and is compatible with the description (106) of its boundary.

We clarify the meaning of compatibility with (106) during the proof.

Proof. Once we clarify the meaning of the compatibility with (106), the lemma follows from (relative version of) Theorem 3.1, by an induction on $\omega(\beta)$ and $k$. (We define triangulation of its zero set at the same time by induction.)

We first study the first term of the right hand side of (106). We consider

$$
\mathcal{M}_{k_{1}+1}\left(\beta_{1} ; P_{i}, \ldots, P_{i+k_{1}-1}\right) .
$$

It corresponds to the first factor of the first term of the right hand side of (106). We fixed a global section of it already. A triangulation of its zero set is also taken already. So using $e v_{0}$ we have smooth singular chains $P_{1}^{\prime}, \ldots, P_{a}^{\prime}$ of $L$ such that

$$
P_{1}^{\prime}+\cdots+P_{a}^{\prime}=\mathfrak{m}_{k_{1}, \beta_{1}}\left(P_{i}, \ldots, P_{i+k_{1}-1}\right) .
$$

(In case $R$ is not characteristic 2 we need an appropriate sign.) We next consider

$$
\mathcal{M}_{k_{2}+1 ; 1}\left(\beta_{2} ; P_{1}, \ldots, P_{i-1}, P_{j}^{\prime}, P_{i+k_{1}}, \ldots, P_{k}\right) .
$$


The sum of these spaces over $j=1, \ldots, a$ corresponds to the first term of the right hand side of (106). By induction hypothesis a global section of (107) and the triangulation of its zero set are already taken.

We next study the second term of the right hand side of (106). We consider

$$
\mathcal{M}_{k_{2}+1}\left(\beta_{2}\right)_{e v_{1}, \ldots, e v_{i-1}, e v_{i+1}, \ldots, e v_{k_{2}}} \times\left(P_{1} \times \cdots \times P_{i-1} \times P_{i+k_{1}} \times \cdots \times P_{k}\right) .
$$

This corresponds to the second factor of the second term of the right hand side of (106). We rename the boundary marked points such that the $i$-th boundary marked point becomes the 0 -th marked point and that the cyclic order of the boundary marked points is preserved. Then (108) is identified with

$$
\mathcal{M}_{k_{2}+1}\left(\beta_{2} ; P_{i+k_{1}}, \ldots, P_{k}, P_{1}, \ldots, P_{i-1}\right) .
$$

In Sections 13 and 14 we took a global section of this moduli space and a triangulation of its zero set. Together with $e v_{0}$, which is the evaluation map at the renamed 0 -th marked point on the $\beta_{2}$-disc, it defines smooth singular chains $P_{1}^{\prime \prime}, \ldots, P_{a^{\prime}}^{\prime \prime}$ of $L$. We then consider

$$
\mathcal{M}_{k_{1}+1,1}\left(\beta_{1} ; P_{j}^{\prime \prime}, P_{i}, \ldots, P_{i+k_{1}-1}\right) .
$$

By induction hypothesis a global section of (109) and a triangulation of its zero set are already taken. Note (109) corresponds to the second term of the right hand side of (106).

Thus we have found the way how to define the global section of the boundary of $\mathcal{M}_{k ; 1}\left(\beta ; P_{1}, \ldots, P_{k}\right)$. The compatibility at the codimension $\geq 2$ corner can be checked easily. The proof of Lemma 15.9 is now complete.

Definition 15.6. We put

$$
\mathfrak{p}_{k ; \beta}^{\prime}\left(P_{1}, \ldots, P_{k}\right)=\left(\mathcal{M}_{k ; 1}\left(\beta ; P_{1}, \ldots, P_{k}\right), e v_{0}\right) \in S(M ; R) .
$$

Here $\left(S(M ; R), \delta_{M}\right)$ is the smooth singular chain complex with $R$ coefficients. We also denote by $\delta_{M}$ its extension over $\Lambda_{0, \text { nov }}^{R}$ coefficients. We define

$$
\mathfrak{p}_{k}^{\prime}=\sum_{\beta} T^{\omega(\beta)} e^{\mu(\beta) / 2} \mathfrak{p}_{k ; \beta}^{\prime}: B_{k}\left(S\left(L ; \Lambda_{0, \text { nov }}^{R}\right)[1]\right) \rightarrow S\left(M ; \Lambda_{0, \text { nov }}^{R}\right)
$$

and

$$
\mathfrak{p}^{\prime}=\sum_{k} \mathfrak{p}_{k}^{\prime}: C H\left(S\left(L ; \Lambda_{0, \text { nov }}^{R}\right)[1]\right) \rightarrow S\left(M ; \Lambda_{0, \text { nov }}^{R}\right)
$$


Let $i_{!}: S^{k}(L ; R) \rightarrow S^{k+n}(M ; R)$ be the Gysin homomorphism. Then we show the following properties which are analogs of (3.8.10.1) and (3.8.10.2) of [6].

\section{Lemma 15.10.}

$$
\begin{gathered}
\mathfrak{p}_{1}^{\prime} \equiv i_{!} \quad \bmod \Lambda_{0, \text { nov }}^{+, R}, \\
\delta_{M} \circ \mathfrak{p}^{\prime}+\mathfrak{p}^{\prime} \circ \delta^{H}=0 .
\end{gathered}
$$

Proof. (110) is nothing but (3.8.10.1) of [6]. Note that $\mathfrak{p}_{1,0}^{\prime}=\mathfrak{p}_{1,0}=i_{\text {! }}$. (111) is a consequence of Lemma 15.9. In fact, the first term of the right hand side of (106) corresponds to the first and the second terms of (105) and the second term of the right hand side of (106) corresponds to the third and the fourth terms of (105).

We can include the homotopy unit to the story of $\mathfrak{p}^{\prime}$ as follows. Let $\left(C,\left\{\mathfrak{m}_{k}\right\}\right)$ be a filtered $A_{\infty}$ algebra. We put $C^{+}=C \oplus \Lambda_{0, \text { nov }}^{R} \mathbf{e}^{+} \oplus \Lambda_{0, \text { nov }}^{R} \mathbf{f}$. If $C$ has a homotopy unit, we can extend the filtered $A_{\infty}$ structure of $C$ to ones on $C^{+}$such that $\mathbf{e}^{+}$is the exact unit. (See [6] Section 3.4 for the precise definition.) Our $A_{\infty}$ algebra $S\left(L ; \Lambda_{0, \text { nov }}^{R}\right)$ has a homotopy unit. (The proof of this fact is the same as in [7] Section 7.3 using (relative version of) Theorem 3.1.) Now we can use the construction of [7] Subsection 7.4.1 to extend $\mathfrak{p}^{\prime}$ to

$$
\mathfrak{p}^{\prime+}: C H\left(S\left(L ; \Lambda_{0, \text { nov }}^{R}\right)^{+}[1]\right) \rightarrow S\left(M ; \Lambda_{0, \text { nov }}^{R}\right)
$$

that satisfies

$$
\delta_{M} \circ \mathfrak{p}^{\prime+}+\mathfrak{p}^{\prime+} \circ \delta^{H}=0 .
$$

Moreover it satisfies

$$
\mathfrak{p}_{k}^{\prime+}\left(\ldots, \mathbf{e}^{+}, \ldots\right)=0
$$

for $k \neq 1$. We also have $\mathfrak{p}_{1}^{\prime+}\left(\mathbf{e}^{+}\right)=P D[L]$.

Remark 15.7. The $R=\mathbb{Q}$ version of this statement is (3.8.10.4), (3.8.10.5) [6]. We note that $(3.8 .10 .6)[6]$ states $\mathfrak{p}_{2}^{+}\left(x \mathbf{e}^{+}\right)=x$. Actually this is an error. The correct statement is $\mathfrak{p}_{2}^{+}\left(x \mathbf{e}^{+}\right)=0$.

Now we use this operator to prove Theorem $15.3(2)$ and $P D[p t] \notin \operatorname{Im}\left(\delta_{r}\right)$ in Theorem 15.4 (1). Let $b \in \mathcal{M}_{\text {weak }}(L ; R)$. Namely $b \in S\left(L ; \Lambda_{0, \text { nov }}^{R}\right)^{+}$is degree 1 
such that $b \equiv 0 \bmod \Lambda_{0, \text { nov }}^{+, R}$ and $b$ satisfies

$$
\sum_{k} \mathfrak{m}_{k}\left(b^{k}\right) \equiv 0 \quad \bmod \Lambda_{0, \text { nov }}^{+, R} \mathbf{e}^{+} .
$$

We define $\mathfrak{p}_{b}^{\prime+}: S\left(L ; \Lambda_{0, \text { nov }}^{R}\right)^{+}[1] \rightarrow S\left(M ; \Lambda_{0, \text { nov }}^{R}\right)$ by

$$
\mathfrak{p}_{b}^{\prime+}(x)=\mathfrak{p}^{\prime+}\left(x e^{b}\right)
$$

\section{Lemma 15.11.}

$$
\begin{gathered}
\mathfrak{p}_{b}^{\prime+} \equiv i_{!} \quad \bmod \Lambda_{0, \text { nov }}^{+, R}, \\
\mathfrak{p}_{b}^{\prime+} \circ \mathfrak{m}_{1}^{b}+\delta_{M} \circ \mathfrak{p}_{b}^{\prime+}=0 .
\end{gathered}
$$

Proof. Since $b \equiv 0 \bmod \Lambda_{0, \text { nov }}^{+, R}$, (116) follows from (110). To prove (117), we first note that (112) implies

$$
\delta_{M}\left(\mathfrak{p}^{\prime+}\left(x e^{b}\right)\right)+\mathfrak{p}^{\prime+}\left(\mathfrak{m}\left(e^{b} x e^{b}\right) e^{b}\right)+\mathfrak{p}^{\prime+}\left(x e^{b} \mathfrak{m}\left(e^{b}\right) e^{b}\right)=0 .
$$

The first term is $\delta_{M} \circ \mathfrak{p}_{b}^{\prime+}$ by definition. The second term is $\mathfrak{p}_{b}^{++} \circ \mathfrak{m}_{1}^{b}$ by definition. The third term is zero by (113) and (114). Hence the lemma.

The conclusion of Lemma 15.11 is the same as [6] Lemma 6.4.5 (for the case $\mathfrak{b}=0)$. Therefore we can prove Theorem $15.3(3)$ and the claim $P D[p t] \notin \operatorname{Im}\left(\delta_{r}\right)$ in the same way as in [6] Subsection 6.4.2 and Subsection 6.4.3 respectively.

Remark 15.8. We do not know how to generalize the formula [6] (3.8.10.3)

$$
\mathfrak{p}_{1} \circ \mathfrak{m}_{0}(1)+\delta_{M} \circ \mathfrak{p}_{0}(1)+G W_{0,1}(M)(L)=0
$$

to our situation. So we do not know how to generalize [6] Theorem 3.8.11 to $\mathbb{Z}$ or $\mathbb{Z}_{p}$ coefficients. In fact, the proof of $[6](3.8 .10 .3)$ uses the moduli space $\mathcal{M}_{0 ; 1}(\beta)$ of pseudo-holomorphic discs without boundary marked point. An element of this moduli space may have a nontrivial automorphism even in case it does not have a sphere bubble. So the argument of Section 12 does not apply.

\section{REFERENCES}

[1] M. F. Atiyah and R. Bott, A Lefschetz fixed point formula for elliptic complexes I, Ann. of Math. 86 (1967), 374-407.

[2] J. Brylinski, Loop spaces, Characteristic classes and Geometric Quantization, Progress in Math. vol 107, Birkhäuser, Boston, MA, 1993

[3] C.-H. Cho, Non-displaceable Lagrangian submanifolds and Floer cohomology with nonunitary line bundle, J. Geom. Phys. 58 (2008), 1465-1476, arXiv:0710.5454. 
[4] B. Fantechi, L Göttsche, L. Illusie, S. Kleimen, N. Nitsure, and A. Vistoli, Fundamental Algebraic Geometry - Grothendieck's FGA Explained, Math. Surveys and Monographs, vol 123, Amer. Math. Soc. 2005.

[5] K. Fukaya, Cyclic symmetry and adic convergence in Lagrangian Floer theory, Kyoto J. Math. 50 (2010) 521- 590, arXiv:0907.4219.

[6] K. Fukaya, Y.-G. Oh, H. Ohta and K. Ono, Lagrangian Intersection Floer theory - Anomaly and obstructions -, Part I, AMS/IP Studies in Advanced Mathematics, vol 46.1, Amer. Math. Soc./International Press, 2009. MR2553465.

[7] K. Fukaya, Y.-G. Oh, H. Ohta and K. Ono, Lagrangian Intersection Floer theory - Anomaly and obstructions -, Part II, AMS/IP Studies in Advanced Mathematics, vol 46.2, Amer. Math. Soc./International Press, 2009. MR2548482.

[8] K. Fukaya, Y.-G. Oh, H. Ohta and K. Ono, Chapter 8 of the preprint version of [6], [7] can be obtained from http://www.math.kyoto-u.ac.jp/ fukaya/.

[9] K. Fukaya, Y.-G. Oh, H. Ohta and K. Ono, Canonical models of filtered $A_{\infty}$-algebras and Morse complexes, New perspectives and challenges in symplectic field theory, 201-227, CRM Proc. Lecture Notes, 49, Amer. Math. Soc., Providence, RI, 2009.

[10] K. Fukaya, Y.-G. Oh, H. Ohta and K. Ono, Lagrangian Floer theory on compact toric manifolds I, Duke Math. J. 151 (2010), 23-174.

[11] K. Fukaya, Y.-G. Oh, H. Ohta and K. Ono, Lagrangian Floer theory on compact toric manifolds II; Bulk deformations, Selecta Math. (N.S.) 17 (2011), 609-711.

[12] K. Fukaya, Y.-G. Oh, H. Ohta and K. Ono, Anti-symplectic involution and Floer cohomology, submitted, arXiv:0912.2646.

[13] K. Fukaya, Y.-G. Oh, H. Ohta and K. Ono, Displacement of polydisks and Lagrangian Floer theory, J. Symplectic Geom. 11 (2013), 231-268.

[14] K. Fukaya and K. Ono, Arnold conjecture and Gromov-Witten invariants, Topology 38 (1999), 933-1048.

[15] J. Giraud, Cohomologie nonabélienne, Die Grundlehren der Math. Wissenschaften, vol 179, Springer, Berlin, 1970.

[16] M. Goresky, Triangulation of stratified objects, Proc. of Amer. Math. Soc. 72 (1978), 193200 .

[17] A. Grothendieck, Fondements de la Géometrie Algébraique, Extraits du Séminaire Bourbaki 1957- 1962, Springer, Berlin, 1962

[18] A. Grothendieck, Séminaire de Géometrie Algébraic de Bois Marie 1 Révétements étales et groupe fondamental, Lecture Note in Math. vol 224, Springer, Berlin, 1971.

[19] J. Hudson, Piecewise Linear Topology, Benjamin New York, 1969.

[20] J. Mather, Stratifications and mappings, in Dynamical systems, Proc. Sympos. vol 41, pp. 195-232, Univ. Bahia, Salvador, Academic Press New York, 1973.

[21] J. Munkres, Elementary Differential Topology, Annals of Mathematics Studies, vol 54, Princeton University Press, Princeton, 1966.

[22] I. Satake, On a generalization of the notion of manifold, Proc. Nat. Acad. Sci. U.S.A. 42 (1956), 359-363.

[23] J. Whitehead, On $C^{1}$ complexes, Ann. of Math. 41 (1940), 809 - 824. 
[24] H. Whitney, Tangents to analytic variety, Ann. of Math. 81 (1965), 496-549.

Kenji Fukaya

Simons Center for Geometry and Physics, State University of New York, Stony

Brook, NY 11794-3636, USA \& Department of Mathematics, Kyoto University, Kyoto, Japan

E-mail: fukaya@math.kyoto-u.ac.jp

\section{Yong-Geun $\mathrm{Oh}$}

Center for Geometry and Physics, Institute for Basic Sciences (IBS), Pohang, Gyungbuk 790-784, Korea,

Department of Mathematics, POSTECH, Pohang, Korea, \&

Department of Mathematics, University of Wisconsin, Madison WI 53705, USA.

E-mail: oh@math.wisc.edu

Hiroshi Ohta

Graduate School of Mathematics, Nagoya University, Nagoya, 464-8602, Japan \& Korea Institute for Advanced Study, Seoul, Korea

E-mail: ohta@math.nagoya-u.ac.jp

Kaoru Ono

Research Institute for Mathematical Sciences, Kyoto University, Kyoto, 606-8502, Japan \& Korea Institute for Advanced Study, Seoul, Korea

E-mail: ono@kurims.kyoto-u.ac.jp 